\title{
Matter Chern Simons theories in a background magnetic field
}

\author{
Indranil Halder and Shiraz Minwalla \\ Department of Theoretical Physics, Tata Institute of Fundamental Research, \\ Homi Bhabha Rd, Mumbai 400005, India \\ E-mail: indranil.halder@tifr.res.in, minwalla@theory.tifr.res.in
}

AbSTRACT: We study large $N 2+1$ dimensional fermions in the fundamental representation of an $\mathrm{SU}(N)_{k}$ Chern Simons gauge group in the presence of a uniform background magnetic field for the U(1) global symmetry of this theory. The magnetic field modifies the Schwinger Dyson equation for the propagator in an interesting way; the product between the self energy and the Greens function is replaced by a Moyal star product. Employing a basis of functions previously used in the study of non-commutative solitons, we are able to exactly solve the Schwinger Dyson equation and so determine the fermion propagator. The propagator has a series of poles (and no other singularities) whose locations yield a spectrum of single particle energies at arbitrary t' Hooft coupling and chemical potential. The usual free fermion Landau levels spectrum is shifted and broadened out; we compute the shifts and widths of these levels at arbitrary t'Hooft coupling. As a check on our results we independently solve for the propagators of the conjecturally dual theory of Chern Simons gauged large $N$ fundamental Wilson Fisher bosons also in a background magnetic field but this time only at zero chemical potential. The spectrum of single particle states of the bosonic theory precisely agrees with those of the fermionic theory under Bose-Fermi duality.

Keywords: Anyons, Chern-Simons Theories, Non-Commutative Geometry, Quantum Groups

ARXIV EPRINT: 1904.07885 


\section{Contents}

1 Introduction 1

2 Free particles in a magnetic field $\quad 6$

2.1 Non-relativistic particles 6

2.1.1 Symmetries of the Green's function 8

2.1.2 Differential equation for the Green's function 9

2.1.3 Solution for the Green's function 10

2.1.4 Adding spin 11

2.2 Free relativistic bosons in a magnetic field 11

$\begin{array}{lll}2.3 & \text { Free relativistic fermion in a magnetic field } & 12\end{array}$

$\begin{array}{lll}2.4 & \text { Free relativistic particle of spin s in a magnetic field } & 15\end{array}$

3 A twisted convolution (Moyal star product) 16

$\begin{array}{lll}3.1 & \text { Twisted convolution and free propagators } & 17\end{array}$

$\begin{array}{lll}3.1 .1 & \text { Free non-relativistic particles of spin } s & 17\end{array}$

$\begin{array}{lll}3.1 .2 & \text { Free relativistic boson } & 18\end{array}$

$\begin{array}{lll}3.1 .3 & \text { Free relativistic fermion } & 18\end{array}$

4 Regular fermions coupled to Chern Simons gauge fields $\quad 18$

$\begin{array}{lll}\text { 4.1 Gap equation in a uniform background gauge field } & 18\end{array}$

4.2 Solving for $\alpha_{R}$ in terms of $\Sigma_{R} \quad 20$

4.3 Recursion relations for the coefficients of $\Sigma \quad 22$

4.4 Reality properties 23

4.5 The integral over $\omega \quad 24$

4.6 Explicit gap equations 25

4.6.1 Positive band M 26

4.6.2 Positive exceptional band 26

4.6.3 Negative exceptional band 26

$\begin{array}{ll}\text { 4.6.4 Negative band M } & 27\end{array}$

$\begin{array}{lll}4.7 & \text { Solution of the gap equations } & 27\end{array}$

4.7.1 $\mu$ in the M positive/negative band 27

4.7.2 $\mu$ in the positive/negative exceptional band 31

4.8 The $b \rightarrow 0$ limit and the regulated gap equation 32

4.9 Summary of final results for the fermion propagator 34

4.9.1 $\mu$ in the positive/negative M band 34

4.9.2 $\mu$ in the positive/negative exceptional band 35

4.10 Gap equation in bosonic variables in exceptional bands with some choices of signs $\quad 36$

4.10.1 $\zeta_{\mathrm{ex}}, \lambda_{F}$ all negative with $\mu$ in the positive exceptional band 36

4.10.2 $\zeta_{\mathrm{ex}}, \lambda_{F}$ all positive with $\mu$ in the negative exceptional band 37

4.11 The gap equations in bosonic variables in the generic case 38 
4.11.1 $\mu$ in positive $\mathrm{M}$ band, $\lambda_{F}$ negative 38

4.11.2 $\mu$ in negative $\mathrm{M}$ band, $\lambda_{F}$ positive $\quad 39$

4.11.3 $\mu$ in positive M band, $\lambda_{F}$ positive $\quad 40$

4.11.4 $\mu$ in negative $\mathrm{M}$ band, $\lambda_{F}$ negative $\quad 40$

4.12 Solving for $\zeta_{\text {ex }} \quad 41$

4.12.1 Existence of solutions $\quad 41$

4.12.2 Uniqueness of solutions 41

4.12.3 Perturbation theory in small $\lambda_{F} \quad 42$

4.12.4 Perturbation theory in small $\lambda_{B} \quad 44$

4.13 Numerical interpolation between these two regimes 46

5 Regular bosons coupled with Chern Simons gauge field 49

$\begin{array}{lll}5.1 \text { Gap equations } & 50\end{array}$

5.2 Constant magnetic field 53

$\begin{array}{ll}5.3 & \text { Solving the gap equations } \\ 5.4 & \text { Critical }\end{array}$

5.4 Critical scaling limit and the duality 55

5.5 Matching under duality 55

6 Future directions $\quad 56$

$\begin{array}{ll}\text { A Conventions } & 60\end{array}$

$\begin{array}{ll}\text { B Constraints of gauge invariance } & 61\end{array}$

C Spectrum of free particles in a magnetic field $\quad 61$

$\begin{array}{lll}\text { C.1 Boson } & 61\end{array}$

C.2 Fermion 63

$\begin{array}{lc}\text { D Star product and twisted convolution } & 66\end{array}$

$\begin{array}{lll}\text { D.1 Star product basis } & 67\end{array}$

$\begin{array}{lll}\text { D.2 Expanding functions in star product basis } & 68\end{array}$

$\begin{array}{lll}\text { E Fermionic gap equations } & 69\end{array}$

$\begin{array}{ll}\text { F Details of contour integrations } & \mathbf{7 0}\end{array}$

$\begin{array}{lll}\text { F.1 The first integral } & 70\end{array}$

$\begin{array}{lll}\text { F.2 The second integral } & 70\end{array}$

$\begin{array}{lll}\text { F.3 The third integral } & 71\end{array}$

G Details of fermionic recursion relations $\quad 72$

$\begin{array}{ll}\mathrm{H} \text { Bosonic gap equations } & 73\end{array}$

$\begin{array}{lll}\text { I Reduced set of bosonic gap equations } & 75\end{array}$

$\begin{array}{lll}\text { J Zero magnetic field limit } & 77\end{array}$ 


\section{Introduction}

Three dimensional Chern Simons theories coupled to matter fields in the fundamental representation are interesting for at least six related but distinct reasons. First because of the (by now compelling) evidence for a level rank type Bose Fermi duality between pairs of these theories. ${ }^{1}$ Second because these theories are solvable in the large $N$ limit [4]. Third because the all orders large $N$ solution of various quantities (like $\mathrm{S}$ matrices [20-25] and thermal partition functions [4, 26-39]), apart from supplying overwhelming evidence for duality, reveal qualitatively novel dynamics. ${ }^{2}$ Fourth because these theories have interesting conjectured bulk dual descriptions in terms of Vasiliev theory. Fifth because the effective excitations in massive deformations of these theories are non abelian anyons. Sixth because there exists an intricate and rich set of finite $N$ exact - but physically incompletely understood - results (see e.g. [43-46]) for supersymmetric observables in supersymmetric versions of this theory.

In this paper we carry forward the study of matter Chern Simons theories in the large $N$ limit. We focus attention on the simplest and best studied matter Chern Simons theories, namely the so called regular fermion theory (a theory of a single fundamental multiplet of otherwise free fermions interacting with an $\mathrm{SU}\left(N_{F}\right)$ Chern Simons gauge field) and the conjecturally dual so called critical boson theory (the theory of a single multiplet of fundamental Wilson Fisher bosons interacting with a $\mathrm{U}\left(N_{B}\right)$ Chern Simons gauge field). Each of these theories enjoys invariance under a U(1) global symmetry. ${ }^{3}$ In previous work each of these theories has intensively been studied (at leading order at large $N$ but at all orders in the t' Hooft coupling) both in the absence and in the presence of a chemical potential coupling to this gauge field. In this paper we generalize these older studies in a manner we now describe.

As the theories we study enjoy invariance under a U(1) global symmetry, they can be studied in the presence of a background gauge field $a_{\mu}$ that couples to global symmetry current. Turning on a chemical potential $\mu$ for the $\mathrm{U}(1)$ global symmetry is equivalent, in Euclidean space, to studying the theory in the background of an (imaginary) complex constant gauge field in the time direction

$$
a_{3}=i \mu
$$

\footnotetext{
${ }^{1}$ Motivated by the conjectured dual bulk Vasiliev duals of these theories [1-5], the first suggestion that such a duality might exist was made in [4] The papers $[4,6,7]$ also demonstrated that these theories are effectively 'solvable' in the large $N$ limit. The authors of $[8,9]$ combined the results of $[6,7]$ and SchwingerDyson techniques developed in [4]) to find the first quantitative evidence for such a duality and to propose a concrete duality map. Several subsequent large $N$ - and more recently finite $N$ - computations have supplied overwhelming evidence for the duality. A precise version of the duality conjecture was presented in [10-13]. There have been many subsequent studies of finite $N$ versions and generalizations of these dualities; see [14-19] for a very partial list of references.

${ }^{2}$ See also [40-42] for checks of this duality at the level of four point functions of gauge invariant operators.

${ }^{3}$ In the case of the fermionic theory the U(1) global symmetry is the obvious global symmetry that 'completes' $\mathrm{SU}\left(N_{F}\right)$ to $\mathrm{U}\left(N_{F}\right)$. In the case of the bosonic theory, the $\mathrm{U}(1)$ global symmetry is the so called topological symmetry of the $\mathrm{U}(1)$ part of the gauge field.
} 
While a generic background field $a_{\mu}\left(x^{\mu}\right)$ completely breaks the $\mathrm{SO}(3)$ rotational symmetry of our theory, the special choice (1.1) preserves an $\mathrm{SO}(2)$. In this paper we study a generalization of (1.1) that continues to preserve $\mathrm{SO}(2)$. The generalized background field studied in this paper is that of a uniform magnetic field together with a chemical potential, i.e.

$$
\begin{aligned}
& a_{3}=i \mu \\
& a_{j}=-b \frac{\epsilon_{j i} x^{i}}{2} \text { i.e } f_{i j}=b \epsilon_{i j}
\end{aligned}
$$

Working in the background (1.1), it has been demonstrated in several papers (starting with [4]) that the fermion propagator $\alpha$ and the fermion self energy $\Sigma$ in the regular fermion theory obeys the gap equations ${ }^{4}$

$$
\begin{aligned}
& \Sigma(\omega, \vec{x}-\vec{y})=-\frac{N}{2} \gamma^{\mu} \hat{\alpha}(\vec{x}-\vec{y}) \gamma^{\nu} G_{\mu \nu}(\omega, \vec{x}-\vec{y}) \\
& \left(i \omega \gamma^{3}+\gamma^{i} \partial_{i}+m_{F}\right) \alpha(\omega, \vec{x}-\vec{y})+[\Sigma(\omega) \alpha(\omega)](\vec{x}-\vec{y})=-\delta^{2}(\vec{x}-\vec{y})
\end{aligned}
$$

where $[A B]$ denotes the convolution of the two dimensional functions $A$ and $B$ i.e.

$$
[A B]=\int d^{2} w A(\vec{x}-\vec{w}) B(\vec{w}) .
$$

In this paper we demonstrate that the 'reduced' fermion propagator $\alpha_{R}$ and 'reduced' ${ }^{5}$ self energy $\Sigma_{R}$ in the magnetic field background (1.2) obey the strikingly similar gap equation

$$
\begin{aligned}
& \Sigma_{R}(\omega, \vec{x}-\vec{y})=-\frac{N}{2} \gamma^{\mu} \hat{\alpha}_{R}(\vec{x}-\vec{y}) \gamma^{\nu} G_{\mu \nu}(\omega, \vec{x}-\vec{y}) \\
& \left(i \omega \gamma^{3}+D_{i}^{(x-y)} \gamma^{i}+m_{F}\right) \alpha_{R}(\omega, \vec{x}-\vec{y})+\left(\Sigma_{R}(\omega) *_{b} \alpha_{R}(\omega)\right)(\vec{x}-\vec{y})=-\delta^{2}(\vec{x}-\vec{y})
\end{aligned}
$$

While the first lines in (1.3) and (1.5) are simply identical to each other, the second lines in these two equations differ in two ways. First, the ordinary derivative $\partial_{i}$ in the first term in the second line of (1.3) is replaced by $D_{i}$, the background covariant derivative. Second, that the ordinary convolution $[\Sigma \alpha]$ in the second term of the second line of (1.3) turns into the twisted convolution defined by

$$
\left(A *_{b} B\right)(\vec{x})=\int d^{2} w A(\vec{x}-\vec{w}) e^{-i \frac{b}{2} \epsilon_{i j}\left(x^{i}-w^{i}\right)\left(w^{j}\right)} B(\vec{w})
$$

We pause to explore the twisted convolution (1.6) in more detail. The operation (1.6) is the Fourier transform of the familiar Moyal product in momentum space. More explicitly let

$$
\begin{aligned}
& A(\vec{x})=\int \frac{d^{2} k}{(2 \pi)^{2}} \hat{A}(\vec{k}) e^{i \vec{k} \cdot \vec{x}} \\
& B(\vec{x})=\int \frac{d^{2} k}{(2 \pi)^{2}} \hat{B}(\vec{k}) e^{i \vec{k} \cdot \vec{x}}
\end{aligned}
$$

\footnotetext{
${ }^{4}(1.3)$ applies at leading order in the large $N$ limit in a lightcone gauge. The equation has been presented in Fourier space in time but in coordinate space in space. $G_{\mu \nu}$ is the lightcone gauge boson propagator, $\hat{\alpha}$ is the equal time fermion propagator and $m_{F}$ is the bare fermion mass).

${ }^{5}$ See the discussion around (4.8) for what 'reduced' means.
} 
Then

$$
\begin{aligned}
\left(A *_{b} B\right)(\vec{x}) & =\int \frac{d^{2} k}{(2 \pi)^{2}}\left(\hat{A} \star_{b} \hat{B}\right)(\vec{k}) e^{i \vec{k} \cdot \vec{x}} \text { where } \\
\left(\hat{A} \star_{b} \hat{B}\right)(\vec{k}) & =\exp \left[i \frac{b}{2} \epsilon^{i j} \partial_{k_{i}}^{A} \partial_{k_{j}}^{B}\right] \hat{A}(\vec{k}) \hat{B}(\vec{k})
\end{aligned}
$$

In the second line of (1.8) the derivative $\partial_{k_{i}}^{A}$ acts only on the function $\hat{A}$ while the derivative $\partial_{k_{j}}^{B}$ acts only on the function $\hat{B}$. The r.h.s. of the second line of (1.8) defines the associative but non commutative Moyal star product in momentum space. Note that under this product

$$
\left[k_{i}, k_{j}\right]_{\star}=k_{i} \star_{b} k_{j}-k_{j} \star_{b} k_{i}=i b \epsilon_{i j}
$$

The effective non commutativity of momentum expressed by (1.9) is a familiar phenomenon in the presence of a background magnetic field. ${ }^{6}$ Consider the theory of a non relativistic spinless particle of unit charge in a background magnetic field (see (2.32)). While canonical momentum $\pi_{i}$ conjugate to the variable $x^{i}$ is represented by the (non gauge convariant) operator $-i \partial_{i}$, the more physical mechanical momentum $p_{i}=m \frac{d x^{i}}{d t}$ is represented by the (gauge covariant) operator $-i D_{i}=-i\left(\partial_{i}-i a_{i}\right)=-\left(i \partial_{i}+a_{i}\right)$. It follows that

$$
\left[p_{i}, p_{j}\right]=\left[-i D_{i},-i D_{j}\right]=i\left(\partial_{i} a_{j}-\partial_{j} a_{i}\right)=i f_{i j}=i b \epsilon_{i j}
$$

where we have used (1.2) in the last step. Note that (1.10) agrees exactly with (1.9). The familiar and elementary noncommutativity of mechanical momenta in the presence of a uniform magnetic field (1.10) thus supplies an intuitive explanation for the appearance of the momentum space Moyal star product in the gap equation (1.5).

We now return to the gap equations (1.5). The appearance of the Moyal star product in the equations (1.5) allows us to borrow the technology developed in the study of noncommutative solitons almost twenty years ago in e.g. [47, 48] and many subsequent works. ${ }^{7}$ We are able to simplify (1.5) by expanding the fermion propagator and self energy in a basis of functions introduced in [47], and rewriting the gap equations (1.5) as a set of nonlinear equations for the coefficients in such an expansion. Quite remarkably, it turns out to be possible to exactly solve all the equations so obtained in terms of one real variable $\zeta_{\mathrm{ex}}$. $\zeta_{\mathrm{ex}}$, in turn obeys an equation that is completely explicit but is complicated. We are able to analytically solve this single algebraic equation for the single variable $\zeta_{\text {ex }}$ only in perturbation theory (both at small $\left|\lambda_{F}\right|$ and at small $1-\left|\lambda_{F}\right|$ ). At general values of $\lambda_{F}$ we are able to solve for $\zeta_{\mathrm{ex}}$ only numerically. In terms $\zeta_{\mathrm{ex}}$ we have a completely explicit solution for the zero temperature fermion propagator in the background (1.2). We present our derivation for this propagator in section 4; detailed final results for the fermion propagator are summarized in subsection 4.9. In the next few paragraphs we describe some aspects of these results in qualitative terms.

\footnotetext{
${ }^{6}$ We thank N. Seiberg for reminding us of this fact, emphasizing its relevance and for related discussions.

${ }^{7}$ The study of noncommutative solitons was motivated by the demonstration [49] that the dynamics of D branes is governed by non commutative field theories in a particular combined low energy and large field scaling limit [49] and also by the discovery of novel IR effects in such theories at the quantum level [50].
} 
Of course the fermion propagator $\alpha_{R}$ that appears in (1.5) - and that we are largely able to solve for - is not gauge invariant and so is not completely physical. The unambiguously physical information in this propagator lies in its singularities. At least at values of the chemical potential at which we are able to perform our computations completely reliably, these singularities all turn out to be poles that represent on-shell 'one particle states' in the background (1.2). We are once again able to solve for the positions of the poles of this propagator completely explicitly in terms of $\zeta_{\mathrm{ex}}$; once again $\zeta_{\mathrm{ex}}$ is the solution to a completely explicit but complicated equation. Once again we are able to solve for $\zeta_{\text {ex }}$ analytically in perturbation theory, but explicitly numerically. Putting together all this information we are able to completely understand the structure of 'single particle excitations' of our theory about the background (1.2), as we now explain in some detail.

Let us first consider the limit $\lambda_{F}=0$. In this limit the regular fermion theory is free. The single fermion energy spectrum in this limit is simply that of the free Dirac equation in a background magnetic field. As we review in detail below (see around (2.48)) these energy levels are given by

$$
E^{\eta \nu J}=\eta \sqrt{m_{F}^{2}+2 b\left(\nu+\frac{1}{2}-\eta \frac{\operatorname{sgn}\left(m_{F}\right)}{2}\right)}
$$

In (1.11) $\nu$ is a Landau level label that ranges from 0 to $\infty, J$ is an angular momentum label that parameterizes degeneracy within Landau Levels and $\eta= \pm 1$ decides whether the energy levels in question have positive or negative energy. (1.11) differs from the corresponding formula for free minimally coupled scalar in only one way - the term proportional to $\frac{\operatorname{sgn}\left(m_{F}\right)}{2}$ in $(1.11)$ is missing in the simpler scalar case. The reason for this difference is easy to understand; this term in (1.11) has its origins in a B.S coupling (note the spin of a Dirac fermion of mass $m_{F}$ equals $\frac{\operatorname{sgn}\left(m_{F}\right)}{2}$ in $2+1$ dimensions) which is obviously absent for a free scalar particle.

At (absolute value of) energies larger than the (absolute value of) the chemical potential, the exact finite coupling results of this paper for the effective spectrum of single particle states differs from (1.11) in only two ways. First the term $m_{F}^{2}$ that appears in the square root on the r.h.s. of (2.48) is replaced by a coupling constant dependent dynamical mass, whose value is given by the solution to an explicit but complicated equation. Second, the quantity $\frac{\operatorname{sgn}\left(m_{F}\right)}{2}$, which also appears in under the square root on the r.h.s. of $(2.48)$ is replaced by

$$
\frac{\operatorname{sgn}\left(m_{F}\right)}{2} \rightarrow s\left(\lambda_{F}\right)
$$

where

$$
s\left(\lambda_{F}\right)=\frac{\operatorname{sgn}\left(m_{F}\right)-\lambda_{F}}{2}
$$

The replacement (1.12) is simply a consequence of the fact [51] (explained and explored in detail in the upcoming paper [52]) that this free spin is renormalized to $s(\lambda),{ }^{8}$ in the

${ }^{8}$ The exact formula for this effective spin is [51]

$$
s=\frac{\operatorname{sgn}\left(m_{F}\right)}{2}-\frac{c_{2}(F)}{\kappa_{F}}=\frac{\operatorname{sgn}\left(m_{F}\right)}{2}-\frac{N_{F}^{2}-1}{2 N_{F} \kappa_{F}}
$$

where $C_{2}(F)$ is the quadratic Casimir of the fundamental representation of $\mathrm{SU}(N)$ (see subsection 2.6 of [29] for notation). In the large $N$ limit $s$ reduces to the quantity $s\left(\lambda_{F}\right)$ reported in (1.13). 
interacting theory. At zero chemical potential, in other words, the spectrum of particle energies of our theory is essentially that of a 'non interacting spin $s(\lambda)$ particle' with a coupling constant (and magnetic field) dependent mass.

The effective mass that appears in the spectrum of 'single particle states' of each particle, mentioned in the paragraph above, depends on the chemical potential, i.e. on how many Landau levels are filled. There are two particularly interesting values of the chemical potential associated with any given Landau level. First, the lowest chemical potential at which this level is completely filled. Second, the highest chemical potential at which this Landau level is completely unfilled. Unlike in the free theory these two chemical potentials are not equal (roughly speaking this happens because the effective mass is a function of the chemical potential). We call the difference between these two chemical potentials the width of the corresponding Landau Level. This width - which may be either positive or negative - represents the broadening of the Landau level as it is filled. We are able to explicitly compute the width of every Landau level as a function of the coupling constant; our explicit results are presented in (4.124), (4.126) at small $\left|\lambda_{F}\right|$, in (4.134) at small $\left|\lambda_{B}\right|$ and in the graphs presented in subsection 4.13 at arbitrary values of the coupling.

In this paper we are reliably able to compute the Fermion Greens function only outside the width of any given Landau level. This limitation prevents us from studying the (presumably fascinating) detailed physics within a Landau level width in the current paper. As we discuss at some length in the discussion section it may be possible to do better in future work.

It is particularly interesting to investigate the behaviour of our single particle energy spectrum in the limit in which the modulus of the fermionic t'Hooft coupling tends to unity (recall that our theory is conjectured to admit a weakly coupled dual bosonic description in this limit).

When all Landau levels are unfilled, it turns out that the $\lambda_{B} \rightarrow 1$ (taken with $m_{B}^{\text {cri }}$ held fixed) is smooth. Focussing on this case we have used the conjectured dual description of the theory in terms of bosonic variables to obtain a detailed and precise calculational check of our fermionic single particle spectrum. In section 5 we have performed the bosonic Wilson Fisher analogue of the fermionic computations describe above in some detail. The final output of this section is, once again, a spectrum of single particle energies, this time read off from the poles of the bosonic propagator. The final results for this spectrum agree perfectly under duality with the spectrum obtained from the poles of the fermionic propagator providing a rather impressive check of either the conjectured Bose Fermi duality between the regular fermion and critical boson theories or of the computations presented in this paper, according to taste.

The computation of the bosonic propagator is technically complicated in a situation in which some Landau levels are filled, and we have not attempted to solve for the bosonic propagator in this case, leaving it for future work. From the bosonic point of view the filling of a Landau level is presumably associated with the formation of a Bose condensate. Even in the absence of a magnetic field, the solution of the bosonic theory in the presence of a condensate required new tricks, and was successfully carried out only about a year ago $[37,39]$. It would be very interesting to generalize these computations to include the 
effects of a background magnetic field in the presence of filled Landau levels. We hope to return to this problem in the near future.

Although we have not, in this paper, directly used the bosonic description to obtain the spectrum of single particle states in the presence of filled Landau levels, we can translate our fermionic results to bosonic language and so (assuming duality) obtain a detailed prediction for these results (see subsection 4.11). Provided that at least one Landau level is filled we find that the single particle energy levels behave in a rather dramatic manner in the $\left|\lambda_{B}\right| \rightarrow 0$ (see subsection 4.12.4). If we take this limit with $m_{B}^{\text {cri }}$ held fixed as before, we find that the effective single particle squared mass diverges like $\frac{1}{\left|\lambda_{B}\right|}$. Moreover in the limit that $\left|\lambda_{B}\right|$ is small we find that lowest chemical potential at which any given Landau level is completely filled is always greater than the highest chemical potential at which it is completely empty; i.e. the broadening of Landau levels is always positive - and of order $\frac{1}{\left|\lambda_{B}\right|}$ - in this limit.

The divergence of effective masses and widths like $\frac{1}{\left|\lambda_{B}\right|}$ is a dramatic effect which suggests that the ungauged critical boson theory - like its free counterpart - is simply ill defined above a certain value of the chemical potential, and that this run away behaviour is cured by $\left|\lambda_{B}\right|$ coupling effects. A detailed exploration of this phenomenon (preferably from both the bosonic and the fermion viewpoints) is likely to be a very interesting exercise; one that we leave to future work.

This paper has been devoted to the study of particle propagators and their poles - i.e. to the study of effective single particle energy levels - in the presence of a magnetic field. We have not carefully studied the thermodynamics (e.g. charge as a function of chemical potential) in the same background. We hope to return to this very interesting question (as well as to the equally interesting question of generalizing our results to finite temperature) in the future.

\section{Free particles in a magnetic field}

\subsection{Non-relativistic particles}

Consider the non relativistic Schrodinger equation for a spin-less particle of mass $|M|$ in a uniform magnetic field $b$ described by Hamiltonian

$$
H=-\frac{\left(\nabla_{i}-i a_{i}\right)^{2}}{2|M|}
$$

We work in the in a rotationally invariant gauge

$$
a_{j}=-b \frac{\epsilon_{j i} x^{i}}{2}
$$

All through this paper we restrict to the special case of $b>0$ (the case $b<0$ can be obtained from $b>0$ by a parity transformation).

The eigenstates and eigen energies of this problem are labelled by two integers $\nu$ and $l$.

$$
\nu=0 \ldots \infty
$$


is a 'which Landau Level' label. $l=-\nu \ldots \infty$ labels the angular momentum of states. The eigen functions $\phi_{\nu, m}$ are given by (See C.1 for details)

$$
\phi_{\nu, l}=i^{|l|}\left[\frac{b}{2 \pi} \frac{\left(\nu-\frac{|l|}{2}+\frac{l}{2}\right) !}{\left(\nu+\frac{|l|}{2}+\frac{l}{2}\right) !}\right]^{1 / 2} e^{i l \phi} e^{-u / 2} u^{|l| / 2} L_{\nu-\frac{|l|}{2}+\frac{l}{2}}^{|l|}(u), E_{\nu, l}=\frac{b}{|M|}\left(\nu+\frac{1}{2}\right)
$$

More explicitly, for $l>0,{ }^{9}$

$$
\phi_{\nu, l}=i^{l}\left[\frac{b}{2 \pi} \frac{(\nu) !}{(\nu+l) !}\right]^{1 / 2} e^{i l \phi} e^{-u / 2} u^{l / 2} L_{\nu}^{l}(u)=\sqrt{\frac{2 \pi}{b}} e_{\nu, \nu+l}
$$

and for $l \leq 0$

$$
\phi_{\nu, l}=i^{-l}\left[\frac{b}{2 \pi} \frac{(\nu+l) !}{(\nu) !}\right]^{1 / 2} e^{i l \phi} e^{-u / 2} u^{-l / 2} L_{\nu+l}^{-l}(u)=\sqrt{\frac{2 \pi}{b}} e_{\nu, \nu+l}
$$

Where we have defined following wave functions

$$
e_{m, n}(u, \phi)=i^{m-n} \frac{b}{2 \pi}\left(\frac{n !}{m !}\right)^{1 / 2} e^{-i(m-n) \phi} u^{\frac{m-n}{2}} e^{-\frac{u}{2}} L_{n}^{m-n}(u)
$$

Above map between wave functions can be compactly rewritten as

$$
\phi_{\nu, l}=\sqrt{\frac{2 \pi}{b}} e_{m, n}, \quad m=\nu, \quad n=\nu+l
$$

Note that, with this labelling, the variables $m, n$ range over the values

$$
n, m=0,1 \ldots
$$

Note, of course, that the wave functions $\phi_{\nu, J}$ - and so $e_{n, m}$ - are orthonormal to each other and have been normalized to obey in the usual quantum mechanical sense

$$
\int d^{2} x \frac{2 \pi}{b} e_{n, m}^{*}(\vec{x}) e_{n^{\prime}, m^{\prime}}(\vec{x})=\delta_{n n^{\prime}} \delta_{m m^{\prime}}
$$

In the rest of this subsection we will determine the Greens function of the free non relativistic particle, i.e. we will find the function $\alpha$ that obeys the equation ${ }^{10}$

$$
\left(\partial_{t}-\frac{\left(\partial_{i}^{x}-i a_{i}(x)\right)^{2}}{2|M|}\right) \alpha\left(x, x^{\prime}\right)=\delta^{3}\left(x-x^{\prime}\right)
$$

where the derivative $\partial_{t}, \partial_{i}$ acts on the first argument of the Greens function.

\footnotetext{
${ }^{9}$ Here to obtain the second equality we are using following property

$$
i^{l}\left[\frac{(\nu) !}{(\nu+l) !}\right]^{1 / 2} u^{l / 2} L_{\nu}^{l}(u)=i^{-l}\left[\frac{(\nu+l) !}{(\nu) !}\right]^{1 / 2} u^{-l / 2} L_{\nu+l}^{-l}(u) .
$$
}

${ }^{10}$ Here $i$ runs over space variables 1,2 . 


\subsubsection{Symmetries of the Green's function}

A uniform magnetic field

$$
a_{3}=0, \quad \partial_{i} a_{j}-\partial_{j} a_{j}=\epsilon_{i j} b \quad(i, j=1,2), \quad \epsilon_{12}=1
$$

is invariant under both translations in the (12) plane as well as rotations in this plane. ${ }^{11}$ These symmetries of the gauge invariant field strength are obscured by the fact that theres is no choice of background gauge that preserves all three symmetries. To deal with this complication, we will find it useful to enumerate three gauges, chosen so that each gauge that preserves any one of three spacetime symmetries listed above but breaks the other two.

In this paper we always work in the rotational gauge $g_{r}$

$$
a_{j}=-b \frac{\epsilon_{j i} x^{i}}{2},
$$

which preserves the symmetry of rotations in the (12) plane but breaks the two translational symmetries. The $x_{1}$ translational gauge $g_{1}$

$$
a_{1}=-b x^{2}, \quad a_{2}=0
$$

preserves translations in the $x^{1}$ direction but breaks translations in $x^{2}$ and rotations. Finally the $x_{2}$ translational gauge $g_{2}$

$$
a_{2}=b x^{1}, \quad a_{1}=0
$$

preserves translations in the 2 direction but breaks translations in $x^{1}$ and rotations. Note that

$$
\begin{aligned}
& a_{i}^{g_{1}}=a_{i}^{g_{r}}-\partial_{i}\left(\frac{b x^{1} x^{2}}{2}\right) \\
& a_{i}^{g_{2}}=a_{i}^{g_{r}}+\partial_{i}\left(\frac{b x^{1} x^{2}}{2}\right)
\end{aligned}
$$

Under a gauge transformation of the background field $a_{\mu}$, the propagator $\alpha$ and the self energy transforms as

$$
\begin{aligned}
a_{\mu}(x) & \rightarrow a_{\mu}(x)+\partial_{\mu} \chi(x) \\
\alpha(x, y) & \rightarrow e^{i \chi(x)} \alpha(x, y) e^{-i \chi(y)} \\
\Sigma(x, y) & \rightarrow e^{i \chi(x)} \Sigma(x, y) e^{-i \chi(y)}
\end{aligned}
$$

In this paper we choose to work in the gauge $g_{r}$. In this gauge all Greens functions $\alpha(x, y)$ (and later in this paper self energies $\Sigma(x, y)$ ) are rotationally invariant. It also follows from (2.16) and (2.15) that

$$
e^{i \frac{b x^{1} x^{2}}{2}} \alpha(x, y) e^{-i \frac{b y^{1} y^{2}}{2}}, \quad e^{i \frac{b x^{1} x^{2}}{2}} \Sigma(x, y) e^{-i \frac{b y^{1} y^{2}}{2}}
$$

\footnotetext{
${ }^{11}$ Of course translations in the 3 direction are trivially preserved and are ignored through this discussion.
} 
are both translationally invariant in the 1 direction and so are functions only of $x^{1}-y^{1}$ while

$$
e^{-i \frac{b x^{1} x^{2}}{2}} \alpha(x, y) e^{i \frac{b y^{1} y^{2}}{2}}, \quad e^{-i \frac{b x^{1} x^{2}}{2}} \Sigma(x, y) e^{i \frac{b y^{1} y^{2}}{2}},
$$

are both translationally invariant in the 2 direction and so are function only of $x^{2}-y^{2}$. It follows that both these conditions are met if and only if

$$
\alpha(x, y)=e^{-i \frac{b}{2}\left(x^{1} y^{2}-x^{2} y^{1}\right)} \alpha_{R}(x-y), \quad \Sigma(x, y)=e^{-i \frac{b}{2}\left(x^{1} y^{2}-x^{2} y^{1}\right)} \Sigma_{R}(x-y)
$$

where the functions $\alpha_{R}(x-y)$ and $\Sigma_{R}(x-y)$ are both simultaneously rotationally and translationally invariant. ${ }^{12}$

The phase factor that occurs in (2.17) has a simple physical interpretation. Consider the integral

$$
I(x, y)=\int_{x}^{y} a_{i} d x^{i}
$$

where $a_{i}$ is the background listed in (2.12) and the integral on the r.h.s. is take along the straight line from $x$ to $y$. It is easily verified that

$$
I(x, y)=b \frac{x^{1} y^{2}-x^{2} y^{1}}{2}
$$

so that the Wilson line $e^{i I(x, y)}=e^{i b \frac{x^{1} y^{2}-x^{2} y^{1}}{2}}$. It follows from (2.17) that

$$
\alpha_{R}(\vec{x}-\vec{y})=\alpha(x, y) e^{i I(x, y)}
$$

Under a gauge transformation

$$
\psi(x) \rightarrow e^{i \theta(x)} \psi(x), \quad a_{i}(x) \rightarrow a_{i}(x)+\partial_{i} \theta(x), \quad I(x, y) \rightarrow I(x, y)+\theta(y)-\theta(x)
$$

So that

$$
\alpha(x, y) \rightarrow e^{i \theta(x)} \alpha(x, y) e^{-i \theta(y)}, \quad e^{i I(x, y)} \rightarrow e^{-i \theta(x)} e^{i I(x, y)} e^{i \theta(y)}
$$

It follows that $\alpha_{R}(x, y)=\alpha(x, y) e^{i I(x, y)}$ is background gauge invariant- and consequently both rotationally and translationally invariant. ${ }^{13}$ In words, $\alpha_{R}$ is simply the fermion propagator dressed by the straight line Wilson line that is needed to make it background gauge invariant, and is a natural object to study in the presence of a nontrivial background gauge field.

\subsubsection{Differential equation for the Green's function}

Note that the first of (2.17) can be rewritten as

$$
\alpha(x, y)=e^{i x^{i} a_{i}(y)} \alpha_{R}(x-y)
$$

where $a^{j}(y)$ given in (2.12). It follows that

$$
\partial_{i} \alpha=e^{i \epsilon_{i j} x^{i} a^{j}(y)}\left(\partial_{i}+i a_{i}(y)\right) \alpha_{R}(x-y)
$$

\footnotetext{
${ }^{12}$ Note that the 'dressing factors' that relate $\alpha(x, y)$ to $\alpha_{R}(x, y)$ (and similarly for $\Sigma$ ) are rotationally invariant.

${ }^{13}$ We thank A. Gadde for a discussion on this point.
} 
It follows that the defining equation for the Greens function (2.10) can be rewritten as

$$
\left(\partial_{t}-\frac{\left(\partial_{x_{i}}-i a_{i}\left(x-x^{\prime}\right)\right)^{2}}{2|M|}\right) \alpha_{R}\left(x-x^{\prime}\right)=\delta^{3}\left(x-x^{\prime}\right)
$$

In $(2.24) x$ is the collective notation for $(\vec{x}, t)$ and similarly for $x^{\prime}$ and we have used the fact that

$$
e^{i x^{i} a_{i}\left(x^{\prime}\right)} \delta^{3}\left(x-x^{\prime}\right)=\delta^{3}\left(x-x^{\prime}\right) .
$$

Note that the differential operator that appears on the l.h.s. of (2.24) depends only on $x-x^{\prime}$, consistent with the result, derived in the previous subsection, that $\alpha_{R}$ is a function only of $x-x^{\prime}$.

\subsubsection{Solution for the Green's function}

It is useful to Fourier transform the invariant Greens function in time. Let

$$
\alpha_{R}(x)=\int \frac{d \omega}{2 \pi} e^{i \omega x^{3}} \alpha_{R}(\omega, \vec{x})
$$

It follows in the usual way that

$$
\begin{aligned}
\alpha_{R}(\omega, \vec{x}-\vec{y}) & =\sum_{\nu, l} \frac{\phi_{\nu, l}(\vec{x}) \phi_{\nu, l}^{*}(\vec{y})}{i \omega+E_{\nu}} \\
E_{\nu} & =\frac{b}{|M|}\left(\nu+\frac{1}{2}\right) .
\end{aligned}
$$

where the complete set of wave functions $\phi_{\nu, l}$ were defined in (2.3). In the equation above, $\nu$ ranges from 0 to infinity while $l$ ranges over the range $-\nu,-\nu+1, \ldots$ As the denominator in (2.26) is a function only of $\nu$, it follows that (2.26) can be rewritten as

$$
\alpha_{R}(\omega, \vec{x}-\vec{y})=\sum_{\nu=0}^{\infty}\left(\frac{\sum_{l} \phi_{\nu, l}(\vec{x}) \phi_{\nu, l}^{*}(\vec{y})}{i \omega+E_{\nu}}\right)
$$

As $\alpha_{R}(\omega, \vec{x}-\vec{y})$ is a function only of the difference between its spatial positions for every value of $\omega$, it follows that the same must be true of residue of the $\alpha_{R}$ at $\omega=-i E_{\nu}$. It follows, in other words, that the quantity

$$
\sum_{l} \phi_{\nu, l}(\vec{x}) \phi_{\nu, l}^{*}(\vec{y})
$$

is translationally invariant and so

$$
\alpha_{R}(\omega, \vec{x})=\sum_{\nu=0}^{\infty}\left(\frac{\sum_{l} \phi_{\nu, l}(\vec{x}) \phi_{\nu, l}^{*}(0)}{i \omega+E_{\nu}}\right)
$$

However the wave function $\phi_{\nu, l}(u, \phi)$, like any regular wave function at angular momentum $l$, behaves at small values of $u$ like $u^{|l|}$ (times, possibly, a positive power of $u$ ) and so vanishes at the origin unless $l=0$. So it follows that

$$
\alpha_{R}(\omega, \vec{x})=\sum_{\nu=0}^{\infty} \frac{\phi_{\nu, 0}(\vec{x}) \phi_{\nu, 0}^{*}(0)}{i \omega+E_{\nu}}
$$


or equivalently, using (2.6)

$$
\alpha_{R}(\omega, \vec{x})=\frac{2 \pi}{b} \sum_{n=0}^{\infty} \frac{e_{n, n}(\vec{x}) e_{n, n}^{*}(0)}{i \omega+E_{n}}
$$

or, using $L_{n}(0)=1$ so that $e_{n, n}(0)=\frac{b}{2 \pi}(\operatorname{see}(\mathrm{D} .11))$

$$
\alpha_{R}(\omega, \vec{x})=\sum_{n=0}^{\infty} \frac{e_{n, n}(\vec{x})}{i \omega+E_{n}}
$$

\subsubsection{Adding spin}

Upto this point we have studied the dynamics of a spin-less particle; however the discussion above is easily generalized to include the effects of spin. In $2+1$ dimensions the little group of a massive particle is $\mathrm{SO}(2)$; the irreducible representations of the covering group of $\mathrm{SO}(2)$ are all one dimensional, and are labelled by a continuous spin parameter $s$ (defined so that the phase corresponding to a rotation of angle $\alpha$ is $e^{1 \alpha s}$.). The Schrodinger equation for a spin $s$ particle on an arbitrary manifold differs from the corresponding equation for a spin-less particle even in the absence of a magnetic field (the derivative is generalized to a covariant derivative including the spin connection). In the special case of flat space studied in this paper this difference goes away, and the spin of the particle enters its Schrodinger equation only through a $B . \mu$ coupling in the Hamiltonian. This coupling modifies the Hamiltonian (2.32) to

$$
H=-\frac{\left(\nabla_{i}-i a_{i}\right)^{2}}{2|M|}-\frac{b g s}{2|M|}
$$

where $s$ is the particle's spin and $g$ is the ' $\mathrm{g}$ ' factor - the anomalous magnetic moment of the particle (the quantity that is 2 for a relativistic fermion). The wave functions $(2.3)$ remain unchanged, but their eigen energies are now given by

$$
E_{\nu}=\frac{b}{|M|}\left(\nu+\frac{1}{2}-\frac{g s}{2}\right)
$$

The Greens function continues to take the form (2.31) with $E_{\nu}$ is given by (2.64).

\subsection{Free relativistic bosons in a magnetic field}

The free Klein Gordon equation in the presence of a background field is given by

$$
-\left(\partial_{t}^{2}+\left(\partial_{i}^{x}-i a_{i}(x)\right)^{2}\right) \phi+M^{2} \phi=0,
$$

and the corresponding propagator obeys the equation

$$
\left(-\left(\partial_{t}^{2}+\left(\partial_{i}^{x}-i a_{i}(x)\right)^{2}\right)+M^{2}\right) \alpha_{R}(x)=\delta^{3}(x)
$$

The eigen solutions to (2.34) are given by

$$
\phi=\phi_{\nu, l} e^{ \pm i t \sqrt{M^{2}+2 b\left(\nu+\frac{1}{2}\right)}}
$$


(recall we are in Euclidean space). The Greens function $\alpha_{R}$ is given by

$$
\begin{aligned}
\alpha_{R}(\omega, \vec{x}) & =\sum_{n=0}^{\infty} \frac{e_{n, n}(\vec{x})}{\omega^{2}+M^{2}+2 b\left(n+\frac{1}{2}\right)} \\
& =\sum_{n=0}^{\infty} \frac{e_{n, n}(\vec{x})}{2 \sqrt{M^{2}+2 b\left(n+\frac{1}{2}\right)}}\left(\frac{1}{i \omega+\sqrt{M^{2}+2 b\left(n+\frac{1}{2}\right)}}+\frac{1}{-i \omega+\sqrt{M^{2}+2 b\left(n+\frac{1}{2}\right)}}\right)
\end{aligned}
$$

Upon setting $\omega=-i|M|+\omega_{N R}$ and taking the non relativistic limit $M \rightarrow \infty$ at fixed $\omega_{N R}$ we find that the propagator (2.37) reduces to the propagator (2.31) (upto proportionality constants), as expected on general grounds.

\subsection{Free relativistic fermion in a magnetic field}

Consider a free Dirac particle governed by the action

$$
S=\int d^{3} x \bar{\psi}\left(\gamma^{\mu} D_{\mu}^{x}+m_{F}\right) \psi
$$

placed in a uniform magnetic field. The equation of motion for this particle

$$
\left(\gamma^{\mu} D_{\mu}+m_{F}\right) \psi=0
$$

can be cast into the Schrodinger form ${ }^{14}$

$$
\left(\partial_{3}+H\right) \psi=0
$$

with

$$
H=\gamma^{3} \gamma^{i} D_{i}+\gamma^{3} m_{F}
$$

The operator $H$ is simply the Hamiltonian of the Minkowskian Dirac wave equation. ${ }^{15}$ As usual, the Hamiltonian $H$ - viewed as a differential operator that acts on spinors that are functions of $x^{1}$ and $x^{2}$ - is Hermitian under the standard inner product

$$
(\chi, \psi)=\int d^{2} x \chi^{\dagger}(\vec{x}) \psi(\vec{x})
$$

Let $\psi_{n}$ denote the normalized eigenfunctions of the operator $H$ with eigenvalue $E_{n}$, i.e.

$$
H \psi_{n}(\vec{x})=E_{n} \psi_{n}(\vec{x})
$$

On general eigenfunctions provide an orthonormal basis for (two dimensional) spinor space and so obey the completeness relation

$$
\sum_{n} \psi_{n}(\vec{x}) \psi_{n}^{\dagger}\left(\overrightarrow{x^{\prime}}\right)=I \delta^{2}\left(\vec{x}-\vec{x}^{\prime}\right)
$$

where $I$ is the identity matrix in $2 \times 2$ spinor space.

\footnotetext{
${ }^{14}$ Note that the operator $\gamma^{\mu} D_{\mu}$ has no good Hermiticity properties. For this reason it is not useful to solve (2.40) by expanding in eigen solutions of the operator $\gamma^{\mu} D_{\mu}+m_{F}$.

${ }^{15} \mathrm{In}$ other words the i.e. the Minkowskian Dirac equation is
}

$$
i \partial_{t} \psi=H \psi \text {. }
$$


We now describe the explicit form of the eigenfunctions $\psi_{n}$ and their energy eigen spectrum. Let us start with the energy spectrum. The spectrum has positive and negative energy states. The energies of positive energy states are given by $E=\xi_{\nu}^{+}$where

$$
\left(\xi_{\nu}^{+}\right)^{2}=m_{F}^{2}+2 b\left(\nu+\frac{1}{2}-\frac{\operatorname{sgn}\left(m_{F}\right)}{2}\right)
$$

The spectrum of negative energy states is given by $E=\xi_{\nu}^{-}$where

$$
\left(\xi_{\nu}^{-}\right)^{2}=m_{F}^{2}+2 b\left(\nu+\frac{1}{2}+\frac{\operatorname{sgn}\left(m_{F}\right)}{2}\right)
$$

These can be combined into the compact expression

$$
E^{\eta \nu J}=\eta \sqrt{m_{F}^{2}+2 b\left(\nu+\frac{1}{2}-\eta \frac{\operatorname{sgn}\left(m_{F}\right)}{2}\right)}
$$

Where $\eta= \pm 1$ for positive/negative energy states. In both (2.46) and (2.47), the parameters $\nu$ range over the values

$$
0,1,2 \ldots \infty
$$

As in previous subsections, in addition to the 'which Landau Level' label, our eigen energies are labelled by their angular momentum $J$. For the positive/negative energy states it is convenient to set

$$
J=l+\eta \frac{\operatorname{sgn}\left(m_{F}\right)}{2}
$$

For both positive and negative energies, $l$ (physically the orbital angular momentum) ranges over the values

$$
-\nu,-\nu+1,-\nu+2 \ldots
$$

Let the eigen functions be denoted by $\psi^{\eta \nu J}$. Recall that $\psi^{\eta \nu J}$ is a two component spinor, whose components we denote by

$$
\psi^{\eta \nu J}=\left[\begin{array}{l}
\psi_{+}^{\eta \nu J} \\
\psi_{-}^{\eta \nu J}
\end{array}\right]
$$

After solving for the eigenfunctions we find following explicit results ${ }^{16}$

$$
\psi_{ \pm}^{\eta \nu J}(u, \phi)=c_{ \pm} \frac{e^{i \operatorname{sgn}(J) l_{ \pm} \phi}}{\sqrt{2 \pi}} u^{\frac{l_{ \pm}}{2}} e^{-\frac{u}{2}} L_{n_{ \pm}}^{l_{ \pm}}(u)
$$

\footnotetext{
${ }^{16} L_{n}^{l}$ is associated Laguerre polynomial. For non-negative integer $n, L_{n}^{l}(x)$ is a polynomial in $x$ of degree $n$ normalised as $L_{n}^{l}(0)=\frac{\Gamma(n+l+1)}{\Gamma(n+1) \Gamma(l+1)}$. For negative integer $n, L_{n}^{l}(x)$ is taken to be zero by definition. In the exceptional range of parameters, the expressions $(2.50)$ involve the quantity $L_{-1}^{l_{ \pm}}$. Consequently the final expressions for $\psi_{ \pm}$listed in (2.51) only involve the functions $L_{n}^{l}(x)$ for $n \geq 0$.
} 
where $\left(u=\frac{b}{2} x_{s}^{2}, \phi\right)$ are coordinates on space and ${ }^{17}$

$$
\begin{aligned}
l_{+} & =\left|J-\frac{1}{2}\right|, l_{-}=\left|J-\frac{1}{2}\right|+\operatorname{sgn}(\mathrm{J}), \mathrm{n}_{+}=\mathrm{n}, \mathrm{n}_{-}=\mathrm{n}-\frac{1}{2} \operatorname{sgn}(\mathrm{J})(1+\operatorname{sgn}(\mathrm{bJ})) \\
l & =J-\eta \frac{\operatorname{sgn}\left(m_{F}\right)}{2}, n=\nu+l \quad(l \leq 0), \quad n=\nu-\eta \frac{\operatorname{sgn}\left(m_{F}\right)}{2}+\frac{1}{2} \quad(l>0) \\
c_{-} & =c_{+}\left(\frac{\sqrt{2|b|}}{m+\operatorname{sgn}(\mathrm{J}) \mathrm{E}^{\eta \nu \mathrm{J}}}\right) \\
c_{+} & =\left(\frac{1}{|b|}\left(\frac{\Gamma\left(1+n_{+}+l_{+}\right)}{\Gamma\left(1+n_{+}\right)}+\left(\frac{c_{-}}{c_{+}}\right)^{2} \frac{\Gamma\left(1+n_{-}+l_{-}\right)}{\Gamma\left(1+n_{-}\right)}\right)\right)^{-1 / 2}
\end{aligned}
$$

In the non relativistic limit the positive energy part (i.e. $\eta>0$ ) of the Dirac equation reduces to a Schrodinger equation for a particle of spin $\frac{m_{F}}{2}$. The effective Schrodinger wave function is proportional to $\psi_{+}$when $m_{F}>0$, but $\psi_{-}$when $m_{F}<0$. Let us consider the two cases in turn. When $m_{F}>0$ it follows from (2.52) that $l_{+}=|l|$ and $n_{+}=\nu$ if $l>0$ or $n_{+}=\nu+l$ if $l \leq 0$. Similarly, it follows from (2.52) that if $m_{F}<0$ then $l_{-}=|l|$ and $n_{-}=\nu$ if $l>0$ and $n_{-}=\nu+l$ if $l \leq 0$. In perfect agreement with the expectations (2.3). The non relativistic limit of (2.46) also agrees perfectly with (2.64) when $g=2$ and $s=\frac{\operatorname{sgn}\left(m_{F}\right)}{2}$.

The Free Dirac Green's function in a background magnetic field satisfies

$$
\left(\gamma^{\mu} D_{\mu}^{x}+m_{F}\right) \alpha_{R}=-\delta^{3}(x)
$$

In order to solve this equation (2.53) we expand

$$
\alpha_{R}\left(x-x^{\prime}\right)=\int \frac{d w}{2 \pi} e^{i \omega\left(t-t^{\prime}\right)} \sum_{n, m} \psi_{n}(\vec{x}) c_{n m}(\omega) \psi_{m}^{\dagger}\left(\overrightarrow{x^{\prime}}\right)
$$

Plugging this expansion into (2.53) and using (2.45)

$$
\int \frac{d w}{2 \pi} \sum_{n, m}\left(i \omega+E_{n}\right) e^{i \omega\left(t-t^{\prime}\right)} \psi_{n}(\vec{x}) c_{n m}(\omega) \psi_{m}^{\dagger}\left(\overrightarrow{x^{\prime}}\right)=-\int \frac{d \omega}{2 \pi} e^{i \omega\left(t-t^{\prime}\right)} \sum_{n} \gamma^{3} \psi_{n}(\vec{x}) \psi_{n}^{\dagger}\left(\overrightarrow{x^{\prime}}\right)
$$

Multiplying both sides of this equation with $\psi_{a}^{\dagger}(\vec{x}), \psi_{b}\left(\overrightarrow{x^{\prime}}\right)$ (respectively from left and right), integrating over $\vec{x}$ and $\overrightarrow{x^{\prime}}$ and using orthonormality we find

$$
c_{n m}(\omega)=\frac{1}{-i \omega-E_{n}} \int d^{2} x \psi_{n}^{\dagger}(\vec{x}) \gamma^{3} \psi_{m}(\vec{x})
$$

Plugging (2.56) into (2.54), and using (2.45) to perform the sum over $m$ we find

$$
\alpha_{R}\left(x-x^{\prime}\right)=i \int_{-\infty}^{\infty} \frac{d w}{2 \pi} e^{i \omega\left(t-t^{\prime}\right)} \sum_{n} \frac{\psi_{n}(\vec{x}) \psi_{n}^{\dagger}\left(\overrightarrow{x^{\prime}}\right) \gamma^{3}}{\omega-i E_{n}}
$$

With explicit form of these eigen functions at hand this equation takes the form

$$
\alpha_{R}\left(x-x^{\prime}\right)=-\int_{-\infty}^{\infty} \frac{d w}{2 \pi} e^{i \omega\left(t-t^{\prime}\right)} \sum_{\nu=0}^{\infty}\left(\sum_{l=-\nu}^{\infty} \frac{\psi^{+\nu l}(\vec{x})\left(\psi^{+\nu l}\right)^{\dagger}\left(\overrightarrow{x^{\prime}}\right) \gamma^{3}}{i \omega+\xi^{+}(\nu)}+\sum_{l=-\nu}^{\infty} \frac{\psi^{-\nu l}(\vec{x})\left(\psi^{-\nu l}\right)^{\dagger}\left(\overrightarrow{x^{\prime}}\right) \gamma^{3}}{i \omega-\xi^{-}(\nu)}\right)
$$

\footnotetext{
${ }^{17}$ Here $|x|$ stands for absolute value of $x$.
} 
As in the discussion around (2.26) the formula (2.54) can be simplified by using the translational invariance of the Greens function $\alpha_{R}$ to set the argument of every occurrence of $\psi^{\dagger}$ to zero. We then use the fact that $\psi^{ \pm \nu l}$ vanish unless $l=0$. It follows that $(2.54)$ can be replaced by

$$
\alpha_{R}\left(x-x^{\prime}\right)=-\int_{-\infty}^{\infty} \frac{d w}{2 \pi} e^{i \omega\left(t-t^{\prime}\right)} \sum_{\nu=0}^{\infty}\left(\frac{\psi^{+\nu 0}(\vec{x})\left(\psi^{+\nu 0}\right)^{\dagger}(0) \gamma^{3}}{i \omega+\xi^{+}(\nu)}+\frac{\psi^{-\nu 0}(\vec{x})\left(\psi^{-\nu 0}\right)^{\dagger}\left(\overrightarrow{x^{\prime}}\right) \gamma^{3}}{i \omega-\xi^{-}(\nu)}\right)
$$

Note that the denominators $\xi^{ \pm}(\nu)$ obey the relationship

$$
\xi_{\nu}^{+}=\xi_{\nu-\operatorname{sgn}\left(m_{F}\right)}^{-}
$$

In other words if we choose

$$
\nu= \begin{cases}\tilde{n}+\frac{1}{2}+\frac{\operatorname{sgn}\left(m_{F}\right)}{2} & + \text { ve energy } \\ \tilde{n}+\frac{1}{2}-\frac{\operatorname{sgn}\left(m_{F}\right)}{2} & \text {-ve energy }\end{cases}
$$

Then we have

$$
\left(\xi_{\nu}^{+}\right)^{2}=\left(\xi_{\nu}^{-}\right)^{2}=m_{F}^{2}+2|b|(\tilde{n}+1) \equiv \xi_{\tilde{n}}
$$

For this reason it is sometimes convenient to combine together the positive and negative energy Landau Levels with the same value of $\tilde{n},{ }^{18}$ upon doing this we find that the propagator is given by

$$
\begin{aligned}
\alpha_{R}^{F}\left(x-x^{\prime}\right) & =\int_{-\infty}^{\infty} \frac{d w}{2 \pi} e^{i \omega\left(t-t^{\prime}\right)} \alpha_{R}^{F}\left(\omega, \vec{x}-\overrightarrow{x^{\prime}}\right) \\
\alpha_{R}^{F}(\omega, \vec{x}) & =\gamma^{+} \alpha_{R,+}^{F}(\omega, \vec{x})+\gamma^{-} \alpha_{R,-}^{F}(\omega, \vec{x})+\gamma^{3} \alpha_{R, 3}^{F}(\omega, \vec{x})+I \alpha_{R, I}^{F}(\omega, \vec{x}) \\
\alpha_{R,+}^{F}(u, \phi) & =-\sum_{n=0}^{\infty} \frac{1}{\Pi(\omega, n)}(i \sqrt{b(n+1)}) e_{n+1, n}(u, \phi) \\
\alpha_{R,-}^{F}(u, \phi) & =-\sum_{n=0}^{\infty} \frac{1}{\Pi(\omega, n)}(-i \sqrt{b(n+1)}) e_{n+1, n}(u, \phi)^{*} \\
\alpha_{R, 3}^{F}(u, \phi) & =-\frac{1}{2} \sum_{n=0}^{\infty}\left(\frac{\left(i \omega-m_{F}\right)}{\Pi(\omega, n-1)}+\frac{\left(i \omega+m_{F}\right)}{\Pi(\omega, n)}\right) e_{n, n}(u, \phi) \\
\alpha_{R, I}^{F}(u, \phi) & =-\frac{1}{2} \sum_{n=0}^{\infty}\left(\frac{\left(i \omega-m_{F}\right)}{\Pi(\omega, n-1)}-\frac{\left(i \omega+m_{F}\right)}{\Pi(\omega, n)}\right) e_{n, n}(u, \phi) \\
-\Pi(\omega, n) & =-\left(i \omega+\xi_{n}\right)\left(i \omega-\xi_{n}\right)=\omega^{2}+m_{F}^{2}+2|b|(n+1)
\end{aligned}
$$

\subsection{Free relativistic particle of spin $\mathrm{s}$ in a magnetic field}

We have already seen that a non relativistic particle of spin $s$ and $g$ factor 2 has a Greens Function with poles located at $\omega=i E_{\nu}$ where

$$
E_{\nu}=\frac{b}{|M|}\left(\nu+\frac{1}{2}-s\right)
$$

\footnotetext{
${ }^{18}$ Note that the levels we combine do not have the same value of $\nu$; their $\nu$ levels differs by unity, as follows from (2.61).
} 
On the other hand we have also seen that the poles of a relativistic particle of spin $s=0$ or $s \pm \frac{1}{2}$ occur at $i \chi_{\nu}^{+}$or $-i \chi_{\nu}^{-}$where

$$
\begin{aligned}
& \left(\chi_{\nu}^{+}\right)^{2}=M^{2}+2 b\left(\nu+\frac{1}{2}-s\right) \\
& \left(\chi_{\nu}^{-}\right)^{2}=M^{2}+2 b\left(\nu+\frac{1}{2}+s\right)
\end{aligned}
$$

It is natural to suggest that the propagator for a 'free particle of arbitrary spin $s$ 'to the extent that one can make sense of such a notion - should also have poles located at the values listed in (2.65). Moreover at these values of $\nu$ we should expect to find an infinite degeneracy of states, with angular momenta given - respectively for positive and negative energy states - by

$$
J=l \pm s
$$

with the 'orbital angular momentum $l$ ' ranging over the values

$$
-\nu,-\nu+1,-\nu+2 \ldots
$$

\section{A twisted convolution (Moyal star product)}

When we turn on interactions in the next section, it will turn out that our matter propagators obey a Schwinger Dyson equation that is given in terms of a twisted convolution $*_{b}$ defined as follows. Given any two functions $A$ and $B$ on $R^{2}$ we define

$$
\left(A *_{b} B\right)(x)=\int d^{2} w A(x-w) e^{-i \frac{b}{2} \epsilon_{i j}\left(x^{i}-w^{i}\right)\left(w^{j}\right)} B(w)
$$

In Fourier space (3.1) is simply the famous Moyal star product. It follows that the $*_{b}$ product is associative but non commutative.

Quite remarkably, the eigen functions $e_{n, m}$ defined above are the basis functions used in the discussions of non-commutative solitons [47] (after flip of position and momentum) and so obey the following identity

$$
\left(e_{n, p} *_{b} \quad e_{p^{\prime}, m}\right)(\vec{x})=\delta_{p p^{\prime}} e_{n, m}(\vec{x})
$$

Now we turn to an algebraic exercise that will be useful in next section. The property (3.2) can be used to define inverse of twisted convolution as we demonstrate below. Consider $2 \times 2$ matrix valued function given by

$$
\alpha=\sum_{m=0}^{\infty}\left(\alpha_{+, m+1} e_{m+1, m} \gamma^{+}+\alpha_{-, m} e_{m, m+1} \gamma^{-}+\alpha_{3, m} e_{m, m} \gamma^{3}+\alpha_{I m} e_{m . m} I\right)
$$

and another similar function given by

$$
\beta=\sum_{m=0}^{\infty}\left(\beta_{+, m+1} e_{m+1, m} \gamma^{+}+\beta_{-, m} e_{m, m+1} \gamma^{-}+\beta_{3, m} e_{m, m} \gamma^{3}+\beta_{I m} e_{m . m} I\right)
$$


Then, using (3.2), it is easily verified that

$$
\left(\alpha *_{b} \beta\right)(\vec{x}-\vec{y})=\left(\beta *_{b} \alpha(\vec{x}-\vec{y})\right)=I \delta^{2}(\vec{x}-\vec{y})
$$

if and only if the coefficients $\alpha_{n}$ are determined in terms of $\beta_{n}$ as follows. For $n>0$

$$
\begin{aligned}
\alpha_{+, n} & =\frac{1}{\Pi(n-1)} \beta_{+, n} \\
\alpha_{-, n} & =\frac{1}{\Pi(n)} \beta_{-, n} \\
\alpha_{3, n} & =\frac{1}{2}\left(\frac{\beta_{3, n-1}-\beta_{I, n-1}}{\Pi(n-1)}+\frac{\beta_{3, n+1}+\beta_{I, n+1}}{\Pi(n)}\right) \\
\alpha_{I, n} & =\frac{1}{2}\left(\frac{\beta_{3, n-1}-\beta_{I, n-1}}{\Pi(n-1)}-\frac{\beta_{3, n+1}+\beta_{I, n+1}}{\Pi(n)}\right)
\end{aligned}
$$

and for $n=0,{ }^{19}$

$$
\begin{aligned}
\alpha_{+, 0} & =0 \\
\alpha_{-, 0} & =\frac{1}{\Pi(0)} \beta_{-, 0} \\
\alpha_{3,0} & =\frac{1}{2}\left(\frac{1}{\beta_{3,0}+\beta_{I, 0}}+\frac{\beta_{3,1}+\beta_{I, 1}}{\Pi(0)}\right) \\
\alpha_{I, n} & =\frac{1}{2}\left(\frac{1}{\beta_{3,0}+\beta_{I, 0}}-\frac{\beta_{3,1}+\beta_{I, 1}}{\Pi(0)}\right)
\end{aligned}
$$

where we have defined

$$
\Pi(n)=\left(\beta_{3, n}-\beta_{I, n}\right)\left(\beta_{3, n+1}+\beta_{I, n+1}\right)+2 \beta_{+, n+1} \beta_{-, n}
$$

\subsection{Twisted convolution and free propagators}

\subsubsection{Free non-relativistic particles of spin $s$}

Consider the function

$$
K_{R}^{N R}(\omega, \vec{x})=\sum_{n=0}^{\infty}\left(i \omega+E_{n}\right) e_{n, n}(\vec{x})
$$

with $E_{n}$ given by (2.64). It follows from (3.2) and (2.31)that

$$
\left(K_{R}^{N R}(\omega) *_{b} \alpha_{R}(\omega)\right)(\vec{x})=\delta^{2}(\vec{x})
$$

(where $\alpha_{R}$ is given by (2.31)). More generally, given any function $A(\omega, \vec{x})$, we have

$$
\left(i \omega-\frac{\left(\partial_{x_{i}}-i a_{i}\left(x-x^{\prime}\right)\right)^{2}}{2|M|}-\frac{b g s}{2|M|}\right) A(\omega, \vec{x})=\left(K_{R}^{N R}(\omega) *_{b} A(\omega)\right)(\vec{x})
$$

In other words, the action of the Schrodinger operator on any wave function can be obtained by star convoluting $K_{R}^{N R}$ with that function.

\footnotetext{
${ }^{19}$ Roughly speaking (3.8) is analytical continuation of (3.6) once we use the definition that

$$
\beta_{+, 0}=0 .
$$
}




\subsubsection{Free relativistic boson}

As in the previous subsection if we define

$$
K_{R}^{B}(\omega, \vec{x})=\sum_{n=0}^{\infty}\left(\omega^{2}+M^{2}+2 b\left(n+\frac{1}{2}\right)\right) e_{n, n}(\vec{x})
$$

it follows that

$$
\left(K_{R}^{B}(\omega) *_{b} \alpha_{R}(\omega)\right)(\vec{x})=\delta^{2}(\vec{x})
$$

(where $\alpha_{R}$ is now the reduced propagator for the relativistic scalar theory given in (2.37)) and, more generally,

$$
\left(\omega^{2}-\left(\partial_{i}^{x}-i a_{i}(x)\right)^{2}+M^{2}\right) A(\omega, \vec{x})=\left(K_{R}^{B}(\omega) *_{b} A(\omega)\right)(\vec{x})
$$

\subsubsection{Free relativistic fermion}

The matrix valued function $K_{R}^{F}$ given by ${ }^{20}$

$$
K_{R}^{F}=\sum_{m=0}^{\infty}\left(i \sqrt{b(m+1)} e_{m+1, m} \gamma^{+}+i \sqrt{b(m+1)} e_{m, m+1} \gamma^{-}+i \omega e_{m, m} \gamma^{3}+m_{F} e_{m . m} I\right)
$$

obeys the identity

$$
\left(K_{R}^{F}(\omega) *_{b} \alpha_{R}(\omega)\right)(\vec{x})=-\delta^{2}(\vec{x})
$$

More generally $K_{R}^{F}$ obeys the identity

$$
\left(\gamma^{3} i \omega+\gamma^{i} D_{i}+m_{F}\right) A(\omega, \vec{x})=\left(K_{R}^{F}(\omega) *_{b} A(\omega)\right)(\vec{x})
$$

where $A$ is any normalizable $2 \times 2$ matrix valued function on $R^{2}$.

\section{Regular fermions coupled to Chern Simons gauge fields}

\subsection{Gap equation in a uniform background gauge field}

Consider $\mathrm{SU}\left(N_{F}\right)$ regular fermions at level $k_{F}-\frac{1}{2} \operatorname{sgn}\left(k_{F}\right) \cdot{ }^{21}$ As usual we use the symbol $\kappa_{F}$ to denote

$$
\kappa_{F}=\operatorname{sgn}\left(k_{F}\right)\left(\left|k_{F}\right|+N_{F}\right)
$$

Our fermionic theory has a U(1) global symmetry - the symmetry under which the fundamental field $\psi$ has charge +1 and the anti-fundamental field $\bar{\psi}$ has charge -1 . In the

\footnotetext{
${ }^{20}$ That is$$
K_{R,+, n}^{F}=i \sqrt{b(n)}, K_{R,-, n}^{F}=i \sqrt{b(n+1)}, K_{R, 3, n}^{F}=i \omega, K_{R, I, n}^{F}=m_{F}
$$

${ }^{21}$ Note that integrating out the fermion shifts the level to $k_{F}-\frac{1}{2} \operatorname{sgn}\left(k_{F}\right)+\frac{1}{2} \operatorname{sgn}\left(m_{F}\right)$. For $\operatorname{sgn}\left(\mathrm{m}_{\mathrm{F}} \mathrm{k}_{\mathrm{F}}\right)=1$ low energy Chern Simons level becomes $k_{F}$, whereas for $\operatorname{sgn}\left(m_{F} k_{F}\right)=-1$ low energy Chern Simons level becomes $\operatorname{sgn}\left(k_{F}\right)\left(\left|k_{F}\right|-1\right)$. At least in absence of a magnetic field, this is consistent with dual bosonic theory being in unhiggsed/higgsed phase (see [37, 39] for more details).
} 
presence of a background gauge field $a_{\mu}$ for this global U(1) symmetry, the Euclidean action for our theory takes the form

$$
S_{F}=\frac{i \kappa_{F}}{4 \pi} \int \operatorname{Tr}\left(A d A+\frac{2}{3} A^{3}\right)+\int \bar{\psi} \gamma^{\mu} \tilde{D}_{\mu} \psi+m_{F} \bar{\psi} \psi
$$

where

$$
\tilde{D}_{\mu} \psi=\left(\partial_{\mu}-i A_{\mu}-i a_{\mu}\right) \psi
$$

Let

$$
\left\langle\psi(x)_{A \alpha} \bar{\psi}^{B \beta}(y)\right\rangle=\delta_{A}^{B} \alpha(x, y)_{\alpha}^{\beta}
$$

( $A$ is an $\mathrm{SU}\left(N_{F}\right)$ fundamental gauge index, - note the subscript $F$ stands for Fermion not flavour $-B$ is an $\mathrm{SU}\left(N_{F}\right)$ gauge anti-fundamental index and $\alpha$ and $\beta$ are spinor indices).

In the large $N$ limit and in the lightcone gauge used in this paper (or more generally in any gauge in which the cubic term drops out of the Chern Simons action) the propagator $\alpha(x, y)$ obeys the 'Schwinger Dyson' or gap equation

$$
\begin{aligned}
& \left(\gamma^{\mu} D_{\mu}^{x}+m_{F}\right) \alpha(x, y)+\int d^{3} w \Sigma(x, w) \alpha(w, y)=-\delta^{3}(x-y) \\
& \Sigma(x, y)=-\frac{N}{2} \gamma^{\mu} \alpha(x, y) \gamma^{\nu} G_{\mu \nu}(x-y)
\end{aligned}
$$

where spinor indices (which contract by the rules of matrix multiplication) have been omitted for simplicity and where

$$
D_{\mu}^{x}=\partial_{\mu}^{x}-i a_{\mu}(x)
$$

and

$$
\left\langle A_{\mu}(x) A_{\nu}(y)\right\rangle=G_{\mu \nu}(x-y)
$$

As we have explained above, in this paper we turn on a uniform magnetic field of magnitude $b$ in the 12 plane and work in the rotationally symmetric gauge (2.12). As explained in subsection 2.1.1 above, the Greens function $\alpha$ and the self energy $\Sigma$ in this gauge are given in terms of translationally and rotationally invariant functions by the formulae (2.17) (which we reproduce here for convenience)

$$
\alpha(x, y)=e^{-i \frac{b}{2}\left(x^{1} y^{2}-x^{2} y^{1}\right)} \alpha_{R}(x-y), \quad \Sigma(x, y)=e^{-i \frac{b}{2}\left(x^{1} y^{2}-x^{2} y^{1}\right)} \Sigma_{R}(x-y)
$$

where $\alpha_{R}(x-y)$ and $\Sigma_{R}(x-y)$ are both simultaneously rotationally and translationally invariant.

Plugging (4.8) into (4.5) yields the following gap equations for $\alpha_{R}(x-y)$ and $\Sigma_{R}(x-y)$.

$$
\begin{aligned}
& \Sigma_{R}(x-y)=-\frac{N}{2} \gamma^{\mu} \alpha_{R}(x-y) \gamma^{\nu} G_{\mu \nu}(x-y) \\
& \left(\gamma^{\mu} D_{\mu}^{(x-y)}+m_{F}\right) \alpha_{R}(x-y)+\int d^{3} w \Sigma_{R}(x-w) e^{-i \frac{b}{2} \epsilon_{i j}\left(x^{i}-w^{i}\right)\left(w^{j}-y^{j}\right)} \alpha_{R}(w-y)=-\delta^{3}(x-y)
\end{aligned}
$$


It is convenient to view our equations in Fourier space in time

$$
\begin{aligned}
\alpha_{R}(x) & =\int \frac{d \omega}{2 \pi} e^{i \omega x^{3}} \alpha_{R}(\omega, \vec{x}) \\
\Sigma_{R}(x) & =\int \frac{d \omega}{2 \pi} e^{i \omega x^{3}} \Sigma_{R}(\omega, \vec{x})
\end{aligned}
$$

For definiteness in this paper we work in $A_{-}(x)=0$ gauge, in which gauge field propagator takes following form

$$
G_{\mu \nu}(x)=-\frac{2 i}{\kappa_{F}} \epsilon_{\mu \nu-} \frac{\delta\left(x^{3}\right)}{x^{+}} \Longrightarrow G_{\mu \nu}(\omega, \vec{x})=-\frac{2 i}{\kappa_{F}} \epsilon_{\mu \nu-} \frac{1}{x^{+}}
$$

It follows from the first of (4.9) that

$$
\Sigma_{R}(x-y) \propto \delta\left(x^{3}-y^{3}\right)
$$

It follows that the factor of $\alpha_{R}(x-y)$ on the r.h.s. of the first of (4.9) is always evaluated at $x^{3}=y^{3}$. Note that the equal time propagator is given in Fourier space by

$$
\left.\alpha_{R}(x-y)\right|_{x^{3}=y^{3}}=\hat{\alpha}_{R}(\vec{x}-\vec{y}) \equiv \int_{-\infty}^{+\infty} \frac{d \omega}{2 \pi} \alpha_{R}(\omega, \vec{x}-\vec{y})
$$

The gap equations can be recast as

$$
\begin{aligned}
& \Sigma_{R}(\omega, \vec{x}-\vec{y})=-\frac{N}{2} \gamma^{\mu} \hat{\alpha}_{R}(\vec{x}-\vec{y}) \gamma^{\nu} G_{\mu \nu}(\omega, \vec{x}-\vec{y}) \\
& \left(i \omega \gamma^{3}+\vec{D}^{(x-y)} \gamma^{i}+m_{F}\right) \alpha_{R}(\omega, \vec{x}-\vec{y})+\left(\Sigma_{R}(\omega) *_{b} \alpha_{R}(\omega)\right)(\vec{x}-\vec{y})=-\delta^{2}(\vec{x}-\vec{y})
\end{aligned}
$$

where $*_{b}$ is the twisted convolution defined in (3.1). It follows immediately from (4.11) (which in particular asserts that $G_{\mu \nu}(\omega, \vec{x})$ is independent of $\omega$ ) that $\Sigma_{R}(\omega, \vec{x})$ is actually independent of $\omega$.

\subsection{Solving for $\alpha_{R}$ in terms of $\Sigma_{R}$}

In this subsection we use the second of (4.14)

$$
\left(i \omega \gamma^{3}+\vec{D}^{(x-y)} \gamma^{i}+m_{F}\right) \alpha_{R}(\omega, \vec{x}-\vec{y})+\left(\Sigma_{R}(\omega) *_{b} \alpha_{R}(\omega)\right)(\vec{x}-\vec{y})=-\delta^{2}(\vec{x}-\vec{y})
$$

to evaluate the propagator $\alpha_{R}$ in terms of $\Sigma_{R}$. Using (3.17) and (3.19) (recall these equations allow us to replace the Dirac differential operator by a star product with the function $\left.K_{R}^{F}\right),(4.15)$ may be rewritten as

$$
\left(K_{R}(\omega) *_{b} \alpha_{R}(\omega)\right)(\vec{x}-\vec{y})=-\delta^{2}(\vec{x}-\vec{y})
$$

where, ${ }^{22}$

$$
\begin{aligned}
& K_{R}(\omega, \vec{x})=K_{R}^{F}(\omega, \vec{x})+\Sigma_{R}(\omega, \vec{x}) \\
& K_{R}^{F}(\omega, \vec{x})=\sum_{m=0}^{\infty}\left(i \sqrt{b(m+1)} e_{m+1, m} \gamma^{+}+i \sqrt{b(m+1)} e_{m, m+1} \gamma^{-}+i \omega e_{m, m} \gamma^{3}+m_{F} e_{m . m} I\right) \\
& \Sigma_{R}(\omega, \vec{x})=\sum_{m=0}^{\infty}\left(\Sigma_{+, m+1} e_{m+1, m} \gamma^{+}+\Sigma_{-, m} e_{m, m+1} \gamma^{-}+\Sigma_{3, m} e_{m, m} \gamma^{3}+\Sigma_{I m} e_{m . m} I\right)
\end{aligned}
$$

\footnotetext{
${ }^{22}$ Recall $\Sigma_{R}(\omega, \vec{x})$ is independent of $\omega$ as argued in previous section.
} 
We will now use (4.16) to solve for $\alpha_{R}$ in terms of the function $K_{R}$ (and so, effectively, in terms of $\Sigma$ ).

We now note that (4.16) is of the form (3.5) with the role of $\beta$ played by $K_{R}$. It follows from (3.6) and (3.8) that for $n>0$

$$
\begin{aligned}
\alpha_{R,+, n} & =-\frac{1}{\Pi(n-1)} K_{R,+, n} \\
\alpha_{R,-, n} & =-\frac{1}{\Pi(n)} K_{R,-, n} \\
\alpha_{R, 3, n} & =-\frac{1}{2}\left(\frac{K_{R, 3, n-1}-K_{R, I, n-1}}{\Pi(n-1)}+\frac{K_{R, 3, n+1}+K_{R, I, n+1}}{\Pi(n)}\right) \\
\alpha_{R, I, n} & =-\frac{1}{2}\left(\frac{K_{R, 3, n-1}-K_{R, I, n-1}}{\Pi(n-1)}-\frac{K_{R, 3, n+1}+K_{R, I, n+1}}{\Pi(n)}\right)
\end{aligned}
$$

and for $n=0$

$$
\begin{aligned}
\alpha_{R,+, 0} & =0 \\
\alpha_{R,-, 0} & =-\frac{1}{\Pi(0)} K_{R,-, 0} \\
\alpha_{R, 3,0} & =-\frac{1}{2}\left(\frac{1}{K_{R, 3,0}+K_{R, I, 0}}+\frac{K_{R, 3,1}+K_{R, I, 1}}{\Pi(0)}\right) \\
\alpha_{R, I, 0} & =-\frac{1}{2}\left(\frac{1}{K_{R, 3,0}+K_{R, I, 0}}-\frac{K_{R, 3,1}+K_{K, I, 1}}{\Pi(0)}\right)
\end{aligned}
$$

where

$$
\Pi(n)=\left(K_{R, 3, n}-K_{R, I, n}\right)\left(K_{R, 3, n+1}+K_{R, I, n+1}\right)+2 K_{R,+, n+1} K_{R,-, n}
$$

The r.h.s. of (4.18) and (4.19) are complicated functions of the unknown coefficients in the expansion of $\Sigma$. We have already seen, however, that these otherwise unknown coefficients are independent of $\omega$. For this reason it is possible to make the $\omega$ dependence in (4.29) (4.18) and (4.19) completely explicit. To proceed we borrow the results (4.28) (which implies the expansion of $\Sigma(4.29)$ ) from the next subsection. Plugging that expansion into (4.18) and (4.19), using the fact that the coefficient functions in (4.29) are independent of $\omega$, and doing a little algebra we find that the quantity $\Pi(n)$ is a quadratic expression in $\omega$; more precisely

$$
\begin{aligned}
\Pi(n)= & -\left(\omega-i \zeta_{+}^{F}(n)\right)\left(\omega+i \zeta_{-}^{F}(n)\right) \\
\zeta_{ \pm}^{F}(n)= & \pm \frac{1}{2}\left(\left(K_{R, I, n+1}-K_{R, I, n}\right) \pm\left(\left(K_{R, I, n+1}-K_{R, I, n}\right)^{2}\right.\right. \\
& \left.\left.+4 K_{R, I, n+1} K_{R, I, n}-8 K_{R,+, n+1} K_{R,-, n}\right)^{1 / 2}\right) \\
= & \pm \frac{1}{2}\left(\left(\Sigma_{R, I, n+1}-\Sigma_{R, I, n}\right) \pm\left(\left(\Sigma_{R, I, n+1}-\Sigma_{R, I, n}\right)^{2}\right.\right. \\
& \left.\left.+4\left(m_{F}+\Sigma_{R, I, n+1}\right)\left(m_{F}+\Sigma_{R, I, n}\right)-8 i \sqrt{b(n+1)}\left(i \sqrt{b(n+1)}+\Sigma_{R,+, n+1}\right)\right)^{1 / 2}\right)
\end{aligned}
$$

We also define the quantity

$$
\zeta_{\mathrm{ex}}=K_{R, I, 0}=m_{F}+\Sigma_{R, I, 0}
$$


The quantities $\zeta_{ \pm}^{F}(n)$ are the positive/negative energy poles of the exact fermion propagator which reduce, in the limit $\lambda_{F} \rightarrow 0$ to the poles $\zeta_{\nu}^{ \pm}$(see (2.46) and (2.47) with the relationship between $\nu$ and $n$ (2.61)) for $n \geq 0$. On the other hand the quantity $\zeta_{\text {ex }}$ reduces to $m_{F}$, i.e. to the energy of the exceptional state at zero $\lambda_{F}$ (see under (2.62)).

The equations (4.18) and (4.19) respectively may now be rewritten as

$$
\begin{aligned}
\alpha_{R,+, n} & =\frac{\left(i \sqrt{b(n)}+\Sigma_{R,+, n}\right)}{\left(\omega-i \zeta_{+}^{F}(n-1)\right)\left(\omega+i \zeta_{-}^{F}(n-1)\right)} \\
\alpha_{R,-, n} & =\frac{i \sqrt{b(n+1)}}{\left(\omega-i \zeta_{+}^{F}(n)\right)\left(\omega+i \zeta_{-}^{F}(n)\right)} \\
\alpha_{R, 3, n} & =\frac{1}{2}\left(\frac{i \omega-m_{F}-\Sigma_{R, I, n-1}}{\left(\omega-i \zeta_{+}^{F}(n-1)\right)\left(\omega+i \zeta_{-}^{F}(n-1)\right)}+\frac{i \omega+m_{F}+\Sigma_{R, I, n+1}}{\left(\omega-i \zeta_{+}^{F}(n)\right)\left(\omega+i \zeta_{-}^{F}(n)\right)}\right) \\
\alpha_{R, I, n} & =\frac{1}{2}\left(\frac{i \omega-m_{F}-\Sigma_{R, I, n-1}}{\left(\omega-i \zeta_{+}^{F}(n-1)\right)\left(\omega+i \zeta_{-}^{F}(n-1)\right)}-\frac{i \omega+m_{F}+\Sigma_{R, I, n+1}}{\left(\omega-i \zeta_{+}^{F}(n)\right)\left(\omega+i \zeta_{-}^{F}(n)\right)}\right)
\end{aligned}
$$

and

$$
\begin{aligned}
\alpha_{R,+, 0} & =0 \\
\alpha_{R,-, 0} & =\frac{i \sqrt{b}}{\left(\omega-i \zeta_{+}^{F}(0)\right)\left(\omega+i \zeta_{-}^{F}(0)\right)} \\
\alpha_{R, 3,0} & =\frac{1}{2}\left(\frac{i}{\omega-i \zeta_{\mathrm{ex}}}+\frac{i \omega+m_{F}+\Sigma_{R, I, 1}}{\left(\omega-i \zeta_{+}^{F}(0)\right)\left(\omega+i \zeta_{-}^{F}(0)\right)}\right) \\
\alpha_{R, I, 0} & =\frac{1}{2}\left(\frac{i}{\omega-i \zeta_{\mathrm{ex}}}-\frac{i \omega+m_{F}+\Sigma_{R, I, 1}}{\left(\omega-i \zeta_{+}^{F}(0)\right)\left(\omega+i \zeta_{-}^{F}(0)\right)}\right)
\end{aligned}
$$

\subsection{Recursion relations for the coefficients of $\Sigma$}

In this subsection we will attempt to complete the process of solving the gap equations by plugging (4.18) and (4.18) into the first of (4.14)

$$
\Sigma_{R}(\vec{x}-\vec{y})=-\frac{N}{2} \gamma^{\mu} \hat{\alpha}_{R}(\vec{x}-\vec{y}) \gamma^{\nu} G_{\mu \nu}(\vec{x}-\vec{y})
$$

to obtain a closed set of equations for the coefficient functions of the quantity $\Sigma$.

As we have noted above, in this paper we follow earlier studies of matter Chern Simons theories (starting with [3]) to work in lightcone gauge $A_{-}=0$. In this gauge we have

$$
G_{\mu \nu}(\vec{x})=-\frac{2 i}{k} \epsilon_{\mu \nu-} \frac{1}{x^{+}}
$$

Note that the only non-zero components of Chern-Simons field propagator are $G_{+3}, G_{3+}$. This immediately implies ${ }^{23}$

$$
\Sigma_{R,-}=0, \Sigma_{R, 3}=0
$$

\footnotetext{
${ }^{23}$ Here we are using the linear algebra identity that for any matrix $\alpha_{R}$ we have

$$
\gamma^{3} \alpha_{R} \gamma^{+}-\gamma^{+} \alpha_{R} \gamma^{3}=2\left(\alpha_{R, I} \gamma^{+}-\alpha_{R, I} I\right)
$$
}


so that the last of (4.17) (the expansion of $\Sigma$ in terms of its components) simplifies to

$$
\Sigma=\sum_{n=0}^{\infty}\left(\Sigma_{R,+, n} e_{n, n-1} \gamma^{+}+\Sigma_{R, I, n} e_{n, n} I\right)
$$

where $\Sigma_{R,+n}$ and $\Sigma_{R,+n}$ are $\omega$ independent numbers.

We now use (D.16) to expand the r.h.s. of (4.25) in a linear sum of the basis elements $e_{n, m}$ using (D.14) and equate coefficients on both sides of (4.25). We obtain the equations

$$
\begin{aligned}
\Sigma_{R, I, n^{\prime}} & =2 i \sqrt{b} \lambda_{F} \sum_{n=n^{\prime}+1}^{\infty} \frac{1}{\sqrt{n}} \hat{\alpha}_{R,-, n-1} \\
\Sigma_{R,+, n^{\prime}+1} & =2 i \sqrt{b} \lambda_{F} \sum_{n=0}^{n^{\prime}} \frac{1}{\sqrt{n^{\prime}+1}} \hat{\alpha}_{R, I, n}
\end{aligned}
$$

where $\hat{\alpha}_{R}$ was defined in (4.13) and as usual we have expanded

$$
\hat{\alpha}=\sum_{m=0}^{\infty}\left(\hat{\alpha}_{+, m+1} e_{m+1, m} \gamma^{+}+\hat{\alpha}_{-, m} e_{m, m+1} \gamma^{-}+\hat{\alpha}_{3, m} e_{m, m} \gamma^{3}+\hat{\alpha}_{I m} e_{m . m} I\right)
$$

Since we have already solved for the coefficients of $\alpha$ - and so the coefficients of $\hat{\alpha}$ - in terms of the coefficients in (4.29), (4.30) are closed equations for the unknown coefficients in the expansion of $\Sigma$ (i.e. the equation (4.29)). We will make these equations more explicit below.

To end this subsection we note that (4.30) is the analogue of the integral equation 2.67 in [3]; as we will see later (4.30) actually reduce to 2.67 of [3] in the zero $b$ limit.

The authors of [3] were able to solve their integral equation by differentiating it (see 2.68 of that paper). In order to solve the equation (4.30) we adopt a similar manoeuver; we simply subtract the equations (4.30) at $n$ and $n-1$ to obtain

$$
\begin{aligned}
\sqrt{n+1}\left(\Sigma_{R, I, n+1}-\Sigma_{R, I, n}\right) & =-2 i \sqrt{b} \lambda_{F} \hat{\alpha}_{R,-, n} \\
\sqrt{n+1} \Sigma_{R,+, n+1}-\sqrt{n} \Sigma_{R,+, n} & =+2 i \sqrt{b} \lambda_{F} \hat{\alpha}_{R, I, n}
\end{aligned}
$$

\subsection{Reality properties}

In this brief subsection we momentarily break the flow of our presentation in order to discuss the reality properties of various quantities. It is not difficult to argue that the quantities

$$
\Sigma_{R, I, n}, \quad K_{R, I, n}, \quad \hat{\alpha}_{R, I, n}, \quad \Pi(n)
$$

are all real, while

$$
\Sigma_{R,-, n}, \quad K_{R, \pm, n}, \quad \hat{\alpha}_{R, \pm, n}
$$

are purely imaginary. In order to see that this is the case we first note that these reality properties are manifest at $\lambda_{F}=0 .{ }^{24}$ We next verify that the reality properties (4.34)

\footnotetext{
${ }^{24}$ It is obvious that (4.33) (4.34) are true of $K_{R}^{F}$. In order to verify the corresponding claims for $\hat{\alpha}_{R}^{F}$ we use (2.63) and perform the integral over $\omega$ needed to obtain $\hat{\alpha}_{R}^{F}$ : note that imaginary terms in the third and fourth lines of (2.63) integrate to zero.
} 
and (4.33) are consistent with our gap equations. The consistency of these reality properties with (4.30) is manifest. In order to verify that (4.34) and (4.33) are consistent with (4.18) we integrate both sides of (4.18) over $\omega$ from $-\infty$ to $\infty$; it is not difficult to convince oneself that the result of such an integral is consistent with the reality properties assumed assigned to the l.h.s. of $(4.18)^{25}$

In this paper we solve our gap equations recursively, order by order in the coupling constant. It follows immediately from the discussion of the last paragraph that each order in the recursive expansion of $\Sigma_{R}$ and $\hat{\alpha}_{R}$ - and hence the full solution - also obey the reality properties (4.34) and (4.33), establishing our claim.

In the rest of this subsection we will solve the recursion relations for the self energy unknowns. We will treat the self energies at generic values of $n$ (i.e., those relevant to the propagator terms (4.18)) and exceptional values of $n$ (i.e. those relevant to the propagator terms (4.19)) separately.

\subsection{The integral over $\omega$}

We now return to the main flow of this section. We will now evaluate the quantities $\hat{\alpha}_{R,-, n}$ and $\hat{\alpha}_{R, I, n}$ by integrating the r.h.s. of (4.23) and (4.19) over $\omega$. We will then insert the resultant expressions into (4.30), turning that equation into a closed equation for the coefficients of $\Sigma$.

The integrals over $\omega$ that we need to evaluate are

$$
I_{1}=\int_{-\infty}^{+\infty} \frac{d \omega}{2 \pi} \alpha_{R,-, n}=\int_{-\infty}^{+\infty} \frac{d \omega}{2 \pi} \frac{i \sqrt{b(n+1)}}{\left(\omega-i \zeta_{+}^{F}(n)\right)\left(\omega+i \zeta_{-}^{F}(n)\right)}
$$

and for $n \neq 0$

$$
\begin{aligned}
I_{2} & =\int_{-\infty}^{+\infty} \frac{d \omega}{2 \pi} \alpha_{R, I, n} \\
& =\int_{-\infty}^{+\infty} \frac{d \omega}{4 \pi}\left(\frac{i \omega-m_{F}-\Sigma_{R, I, n-1}}{\left(\omega-i \zeta_{+}^{F}(n-1)\right)\left(\omega+i \zeta_{-}^{F}(n-1)\right)}-\frac{i \omega+m_{F}+\Sigma_{R, I, n+1}}{\left(\omega-i \zeta_{+}^{F}(n)\right)\left(\omega+i \zeta_{-}^{F}(n)\right)}\right)
\end{aligned}
$$

and also the $n=0$ version of this integral

$$
\tilde{I}_{2}=\int_{-\infty}^{+\infty} \frac{d \omega}{2 \pi} \alpha_{R, I, 0}=\int_{-\infty}^{+\infty} \frac{d \omega}{4 \pi}\left(\frac{i}{\omega-i \zeta_{\mathrm{ex}}}-\frac{i \omega+m_{F}+\Sigma_{R, I, 1}}{\left(\omega-i \zeta_{+}^{F}(0)\right)\left(\omega+i \zeta_{-}^{F}(0)\right)}\right)
$$

The contour for the integral over $\omega$ has so far been taken to the real axis in the complex $\omega$ plane. However there is a natural physical context - one that will be of interest to us in this paper - in which this contour is deformed. This happens when we turn on a chemical

\footnotetext{
${ }^{25}$ The expressions in the r.h.s. of the first two lines of (4.18) are of the schematic form $i A(i \omega)$ while the expressions on the r.h.s. of the third and fourth lines of the same equation take the schematic form $B(i \omega)$ where $A(z)$ and $B(z)$ complex functions that are real valued when $z$ is restricted to the real line. It follows that the integral of the r.h.s. of these equations - performed using any regulation scheme that preserves $\omega \leftrightarrow-\omega$ symmetry (such as the regulation scheme employed in this paper) - is imaginary for the first two lines of (4.18) but real for the next two lines of (4.18), establishing the consistency of (4.18) with the reality properties listed above.
} 
potential $\mu$ for the same global symmetry for which we have turned on a magnetic field (recall this is the symmetry under which all components of $\psi$ carry charge unity while all components of $\bar{\psi}$ carry charge -1 .) Turning on such a chemical potential is equivalent to making the replacement

$$
\partial_{t} \psi \rightarrow\left(\partial_{t}-\mu\right), \quad \partial_{t} \bar{\psi} \rightarrow\left(\partial_{t}+\mu\right) \bar{\psi}
$$

which, in turn, is equivalent to the replacement

$$
\omega \rightarrow \omega+i \mu
$$

in all the formulae above. If we change variables from $\omega$ to $\omega+i \mu$, all the formulae above retain their old form (i.e. the form they had in the absence of the chemical potential) with one single change; the contour of $\omega$ integration, which earlier had been the real axis, changes to the line

$$
\operatorname{Im}(\omega)=i \mu
$$

It follows that in order to solve our problem at arbitrary values of the chemical potential, we need to evaluate the integrals (4.35), (4.36) and (4.37) contours of the form (4.38) at arbitrary values of $\operatorname{Im}(\omega)$. This is easily done; the derivation and results of the relevant integrals are presented in appendix F.

\subsection{Explicit gap equations}

In this subsection we will input the results of the previous subsection into (4.30) in order to turn this equation into a set of closed equations for the components of $\Sigma$. Our final results depend on the value of $\mu$. Through the rest of this paper we assume (F.5). It is useful to introduce some terminology.

If $\zeta_{+}^{F}(m-1)<\mu<\zeta_{+}^{F}(m)$ we say that $\mu$ lies in the $(m-1)^{t h}$ positive band. If $\zeta_{\mathrm{ex}}<\mu<\zeta_{+}^{F}(0)$ we say that $\mu$ lies in the exceptional positive band. If $-\zeta_{-}^{F}(0)<\mu<\zeta_{\mathrm{ex}}$ we say that $\mu$ lies in the exceptional negative band. Finally if $-\zeta_{-}^{F}(m)<\mu<-\zeta_{+}^{F}(m-1)$ we say that $\mu$ lies in the $(m-1)^{t h}$ negative band.

Some notation: we define

$$
\chi_{n}=\frac{1}{\zeta_{+}^{F}(n)+\zeta_{-}^{F}(n)}
$$

The quantity $\chi_{n}$ determines the sum of $\zeta_{ \pm}^{F}(n)$ and so carries less information than the individual values of $\zeta_{+}^{F}(n)$ and $\zeta_{-}^{F}(n)$. However the additional information needed to reconstruct these two quantities individually is obtained quite easily directly from the gap equations. Using (4.21) it follows that

$$
\zeta^{+}(n)-\zeta^{-}(n)=\Sigma_{R, I, n+1}-\Sigma_{R, I, n} .
$$

Now the r.h.s. of (4.40) can be evaluated using (5.24) (and the results for the integral over $\omega$ presented in subsection 4.4. The specific results for $\zeta^{ \pm}(n)$ in terms of $\chi_{n}$ depend on details and will be presented below. 


\subsubsection{Positive band $M$}

To start with let us assume that $\mu$ lies in the $M^{\text {th }}$ positive band with $M \geq 0$ i.e. that $\zeta_{+}(M)<\mu<\zeta_{+}(M+1)$. In this case the gap equations become

$$
\begin{aligned}
\Sigma_{R, I, M+1} & =\Sigma_{R, I, M}=\cdots=\Sigma_{R, I, 1}=\Sigma_{R, I, 0}=-2 \lambda_{F} \sum_{k=M+1}^{\infty} b \chi_{k} \\
\Sigma_{R, I, n^{\prime}+1}= & -2 \lambda_{F} \sum_{n=n^{\prime}+1}^{\infty} b \chi_{n}, \quad\left(n^{\prime} \geq M+1\right) \\
\sqrt{M+1} \Sigma_{R,+, M+1}= & \sqrt{M} \Sigma_{R,+, M}=\cdots=\Sigma_{R,+, 1}=0 \\
\sqrt{M+2} \Sigma_{R,+, M+2}= & \sqrt{b}\left(i \lambda_{F}^{2}\left(\sqrt{b} \chi_{M+1}\right)^{2}-i \lambda_{F} K_{R, I, M+2} \chi_{M+1}+\frac{1}{2} i \lambda_{F}\right) \\
\sqrt{n^{\prime}+1} \Sigma_{R,+, n^{\prime}+1}= & \sqrt{M+2} \Sigma_{R,+, M+2}-\sum_{n=M+2}^{n^{\prime}} \sqrt{b} i \lambda_{F}\left(K_{R, I, n-1} \chi_{n-1}+K_{R, I, n+1} \chi_{n}\right) \\
& +\sum_{n=M+2}^{n^{\prime}} i \sqrt{b} \lambda_{F}^{2}\left(\left(\sqrt{b} \chi_{n}\right)^{2}-\left(\sqrt{b} \chi_{n-1}\right)^{2}\right) \quad\left(n^{\prime} \geq M+2\right)
\end{aligned}
$$

\subsubsection{Positive exceptional band}

In the positive exceptional band (i.e. for $\left.\zeta_{\mathrm{ex}}<\mu<\zeta_{+}(0)\right)$ on the other hand we have

$$
\begin{aligned}
\Sigma_{R, I, n^{\prime}}= & -2 \lambda_{F} \sum_{n=n^{\prime}}^{\infty} b \chi_{n}, \quad(n \geq 0, \text { i.e all } n) \\
\Sigma_{R,+, 1}= & \sqrt{b}\left(i \lambda_{F}^{2}\left(\sqrt{b} \chi_{0}\right)^{2}-i \lambda_{F} K_{R, I, 1} \chi_{0}+\frac{1}{2} i \lambda_{F}\right) \\
\sqrt{n^{\prime}+1} \Sigma_{R,+, n^{\prime}+1}= & \Sigma_{R,+, 1}-\sum_{n=1}^{n^{\prime}} \sqrt{b} i \lambda_{F}\left(K_{R, I, n-1} \chi_{n-1}+K_{R, I, n+1} \chi_{n}\right) \\
& +\sum_{n=1}^{n^{\prime}} i \sqrt{b} \lambda_{F}^{2}\left(\left(\sqrt{b} \chi_{n}\right)^{2}-\left(\sqrt{b} \chi_{n-1}\right)^{2}\right) \quad\left(n^{\prime} \geq 1\right)
\end{aligned}
$$

\subsubsection{Negative exceptional band}

In the negative exceptional band (i.e. $-\zeta_{-}(0)<\mu<\zeta_{\text {ex }}$ ) the equations are

$$
\begin{aligned}
\Sigma_{R, I, n}= & -2 \lambda_{F} \sum_{n}^{\infty} b \chi_{n}, \quad(n \geq 0, \text { i.e all } n) \\
\Sigma_{R,+, 1}= & \sqrt{b}\left(i \lambda_{F}^{2}\left(\sqrt{b} \chi_{0}\right)^{2}-i \lambda_{F} K_{R, I, 1} \chi_{0}-\frac{1}{2} i \lambda_{F}\right) \\
\sqrt{n^{\prime}+1} \Sigma_{R,+, n^{\prime}+1}= & \Sigma_{R,+, 1}-\sum_{n=1}^{n^{\prime}} \sqrt{b} i \lambda_{F}\left(K_{R, I, n-1} \chi_{n-1}+K_{R, I, n+1} \chi_{n}\right) \\
& +\sum_{n=1}^{n^{\prime}} i \sqrt{b} \lambda_{F}^{2}\left(\left(\sqrt{b} \chi_{n}\right)^{2}-\left(\sqrt{b} \chi_{n-1}\right)^{2}\right) \quad\left(n^{\prime} \geq 1\right)
\end{aligned}
$$




\subsubsection{Negative band $M$}

In the $M^{t h}$ negative band for $M \geq 0$ (i.e. $\left.-\zeta_{-}(M+1)<\mu<-\zeta_{-}(M)\right)$ we have

$$
\begin{aligned}
\Sigma_{R, I, M+1}= & \Sigma_{R, I, M}=\cdots=\Sigma_{R, I, 1}=\Sigma_{R, I, 0}=-2 \lambda_{F} \sum_{k=M+1}^{\infty} b \chi_{k} \\
\Sigma_{R, I, n^{\prime}+1}= & -2 \lambda_{F} \sum_{n=n^{\prime}+1}^{\infty} b \chi_{n}, \quad\left(n^{\prime} \geq M+1\right) \\
\sqrt{M+1} \Sigma_{R,+, M+1}= & \sqrt{M} \Sigma_{R,+, M}=\cdots=\Sigma_{R,+, 1}=0 \\
\sqrt{M+2} \Sigma_{R,+, M+2}= & \sqrt{b}\left(i \lambda_{F}^{2}\left(\sqrt{b} \chi_{M+1}\right)^{2}-i \lambda_{F} K_{R, I, M+2} \chi_{M+1}-\frac{1}{2} i \lambda_{F}\right) \\
\sqrt{n^{\prime}+1} \Sigma_{R,+, n^{\prime}+1}= & \sqrt{M+2} \Sigma_{R,+, M+2}-\sum_{n=M+2}^{n^{\prime}} \sqrt{b} i \lambda_{F}\left(K_{R, I, n-1} \chi_{n-1}+K_{R, I, n+1} \chi_{n}\right) \\
& +\sum_{n=M+2}^{n^{\prime}} i \sqrt{b} \lambda_{F}^{2}\left(\left(\sqrt{b} \chi_{n}\right)^{2}-\left(\sqrt{b} \chi_{n-1}\right)^{2}\right) \quad\left(n^{\prime} \geq M+2\right)
\end{aligned}
$$

\subsection{Solution of the gap equations}

\subsection{1 $\mu$ in the $M$ positive/negative band}

To start with let us focus on the gap equations when $\mu$ lies in the $M^{\text {th }}$ positive/negative band with $M \geq 0$.

Solution for $\boldsymbol{\zeta}_{ \pm}^{\boldsymbol{F}}(\boldsymbol{n})$ for $\boldsymbol{n} \geq \boldsymbol{M}+\mathbf{1}$. When $\mu$ lies in either the positive or negative $M^{\text {th }}$ band for $n^{\prime} \geq M+2$ we have

$$
\begin{aligned}
\Sigma_{R, I, n^{\prime}+1}= & -2 \lambda_{F} \sum_{n=n^{\prime}+1}^{\infty} b \chi_{n} \\
\sqrt{n^{\prime}+1} \Sigma_{R,+, n^{\prime}+1}= & \sqrt{M+2} \Sigma_{R,+, M+2}-\sum_{n=M+2}^{n^{\prime}} \sqrt{b} i \lambda_{F}\left(K_{R, I, n-1} \chi_{n-1}+K_{R, I, n+1} \chi_{n}\right) \\
& +\sum_{n=M+2}^{n^{\prime}} i \sqrt{b} \lambda_{F}^{2}\left(\left(\sqrt{b} \chi_{n}\right)^{2}-\left(\sqrt{b} \chi_{n-1}\right)^{2}\right)
\end{aligned}
$$

For $n \geq M+2$ it follows that

$$
\begin{aligned}
\frac{1}{\sqrt{b}}\left(\Sigma_{R, I, n+1}-\Sigma_{R, I, n}\right)= & 2 \lambda_{F} \sqrt{b} \chi_{n} \\
\frac{1}{\sqrt{b}}\left(\sqrt{n+1} \Sigma_{R,+, n+1}-\sqrt{n} \Sigma_{R,+, n}\right)= & -i \lambda_{F}\left(K_{R, I, n-1} \chi_{n-1}+K_{R, I, n+1} \chi_{n}\right) \\
& +i \lambda_{F}^{2}\left(\left(\sqrt{b} \chi_{n}\right)^{2}-\left(\sqrt{b} \chi_{n-1}\right)^{2}\right)
\end{aligned}
$$

Our task is to solve (4.46) for the variables $\Sigma_{R, I, n}$ and $\Sigma_{R,+, n+1}$. The r.h.s. of (4.46) is a rather complicated function of these variables (the complication arrises because we are 
required to substitute (4.21) and (4.39) into this r.h.s. in order to express it in terms of the variables of the problem). Quite remarkably, however, the difference equations (4.46) in fact can be solved exactly. The key to finding the solution of these equations is the observation that (4.46) imply that the quantities

$$
I_{n}=\lambda_{F}^{2}\left(\sqrt{2 b} \chi_{n}\right)^{2}+\frac{1}{\left(\sqrt{2 b} \chi_{n}\right)^{2}}
$$

obey the remarkably simple recursion relation (see appendix $\mathrm{G}$ for a derivation)

$$
I_{n+1}-I_{n}=4 \quad(n \geq M+2)
$$

It follows that

$$
I_{n}=I_{M+2}+4(n-(M-2)) \quad(n \geq M+2)
$$

where $I_{M+2}$ is and as yet arbitrary constant.

In order to obtain (4.48) and (4.49) we used the first of (4.25) for $n^{\prime} \geq M=1$ and the second of (4.46) for $n^{\prime} \geq M+2$. It turns out we can actually do better. Using the fourth lines in (4.41) and (4.44) and also the expressions for $\zeta_{R, I, M+1}$ listed in the first lines of those equations, and proceeding along the lines of appendix $G$, it is possible to show that

$$
I_{n}=I_{M+1}+4(n-(M+1)) \quad(n \geq M+1)
$$

From (4.47) we obtain ${ }^{26}$

$$
\chi_{n}=\frac{1}{\sqrt{2}\left|\lambda_{F}\right| b}\left(\left(c_{F}^{2}+2 b(n+1)\right)-\left(\left(c_{F}^{2}+2 b(n+1)\right)^{2}-\left(\lambda_{F} b\right)^{2}\right)^{1 / 2}\right)^{1 / 2} \quad(n \geq M+1)
$$

where we have traded $I_{M+1}$ for another arbitrary constant $c_{F}$.

The energy eigenvalues $\zeta_{ \pm}^{F}(n)$ can be obtained from $\chi_{n}$ using (4.40). Using the gap equations (third of (4.41) and (4.44), the r.h.s. of (4.40) evaluates to $2 \lambda_{F} b \chi_{n}$ for $n \geq M+1$ i.e.

$$
\zeta^{+}(n)-\zeta^{-}(n)=2 \lambda_{F} b \chi_{n} \quad(n \geq M+1)
$$

and so we find

$$
\begin{aligned}
\zeta_{ \pm}^{F}(n) & =\frac{1}{2}\left( \pm 2 \lambda_{F} b \chi_{n}+\frac{1}{\chi_{n}}\right) \\
\Longrightarrow \zeta_{ \pm}^{F}(n) & =\left[c_{F}^{2}+2 b(n+1) \pm \lambda_{F} b\right]^{1 / 2} \quad(n \geq M+1)
\end{aligned}
$$

${ }^{26}$ More precisely, the quadratic equation that determines $\chi_{n}$ in terms of $I_{n}$ has two solutions

$$
\chi_{n}= \pm \frac{1}{\sqrt{2}\left|\lambda_{F}\right| b}\left(\left(c_{F}^{2}+2 b(n+1)\right) \pm\left(\left(c_{F}^{2}+2 b(n+1)\right)^{2}-\left(\lambda_{F} b\right)^{2}\right)^{1 / 2}\right)^{1 / 2}
$$

We have fixed the sign ambiguity in (4.51) by matching with perturbation theory around $\lambda_{F} \rightarrow 0$. It is possible that the second solution in (4.51) represents a legal solution of the theory, but one that is intrinsically non perturbative in nature. We do not consider this possibility in the current paper, but hope to return to it and other issues in future work. 
We pause to provided a physical interpretation of (4.54). Recall that in the study of the free Dirac equation we employed two distinct (but related) labelling schemes for the energy levels of our system. The physically more transparent labelling was in terms of the variable $\nu$ (in the non relativistic limit this label mapped directly to the usual 'which Landau Level' label). The second, algebraically more convenient scheme - the one also adopted all through this section so far - was to label energy levels by the variable $n . n$ is an algebraically convenient auxiliary label related to $\nu$ via (2.61) which we repeat here for convenience

$$
\nu= \begin{cases}=n+\frac{1}{2}+\frac{\operatorname{sgn}\left(m_{F}\right)}{2} & + \text { ve energy } \\ =n+\frac{1}{2}-\frac{\operatorname{sgn}\left(m_{F}\right)}{2} & \text {-ve energy }\end{cases}
$$

Rewriting the last line of (4.54) in terms of the more physical variable $\nu$ we find (in the appropriate range of $\nu$, i.e. the range that follows from $n \geq M+2$ using (4.55))

$$
\zeta_{ \pm}^{F}(\nu)=\left[c_{F}^{2}+2 b\left(\nu+\frac{1}{2}\right) \mp 2 b s\left(\lambda_{F}\right)\right]^{1 / 2}
$$

where

$$
s\left(\lambda_{F}\right)=\frac{\left(\operatorname{sgn}\left(m_{F}\right)-\lambda_{F}\right)}{2}
$$

is the effective spin of excitations in the regular fermion theory at t' Hooft coupling $\lambda$. It is quite remarkable that (4.56) matches exactly with (2.65) - our naive guess for the spectrum of a theory of 'free particles of spin $s(\lambda)$ ', provided we identify $c_{F}^{2}$ as the squared effective mass our excitation.

Another aside: note also that it follows immediately from (4.54) that

$$
\begin{array}{ll}
\zeta_{+}^{F}(n)^{2}-\zeta_{-}^{F}(n-1)^{2}=2 b\left(1+\lambda_{F}\right) & (n \geq M+2) \\
\zeta_{-}^{F}(n)^{2}-\zeta_{+}^{F}(n-1)^{2}=2 b\left(1-\lambda_{F}\right) & (n \geq M+2)
\end{array}
$$

Note that the r.h.s. of the first of (4.58) vanishes at $\lambda_{F}=-1$ while the r.h.s. of the second of (4.58) vanishes at $\lambda_{F}=+1$. Comparing with (2.61), it follows in particular that when $\left|\lambda_{F}\right|=1$ and $\lambda_{F} m_{F}>0$ then positive and negative energy levels with the same value of $\nu$ are equal and opposite (i.e. the spectrum is symmetric in positive and negative energies when rewritten in terms of $\nu$ ). We will return to this point below.

Solution for $\boldsymbol{\zeta}_{ \pm}^{\boldsymbol{F}}(\boldsymbol{n})$ for $\boldsymbol{n} \leq \boldsymbol{M}$. The gap equations are much easier to solve for $n \leq M$. For these values of $n$ it follows from (4.44) and (4.41) that $\Sigma_{R, I, n}$ and $\Sigma_{R,+, n}$ are both independent of $n$ in this range. It follows immediately that

$$
\begin{aligned}
\zeta_{ \pm}^{F}(n)^{2} & =\left(m_{F}+\Sigma_{R, I, n}\right)^{2}-2 i \sqrt{b(n+1)}\left(i \sqrt{b(n+1)}+\Sigma_{R,+, n+1}\right) \\
& =\left(m_{F}+\Sigma_{R, I, 0}\right)^{2}-2 i \sqrt{b} \Sigma_{R,+, 1}+2 b(n+1) \\
& =\left(m_{F}+\Sigma_{R, I, 0}\right)^{2}+2 b(n+1)
\end{aligned}
$$

Note that in this range (and unlike for the case $n \geq M+1) \zeta_{+}^{F}(n)=\zeta_{-}^{F}(n)$. We remind the reader that

$$
m_{F}+\Sigma_{R, I, 0}=K_{R, I, 0}=\zeta_{\mathrm{ex}}
$$


It follows that (4.59) can be rewritten as

$$
\zeta_{ \pm}^{F}(n)^{2}=\zeta_{\mathrm{ex}}^{2}+2 b(n+1) \quad \text { For } n \leq M
$$

(4.61) is precisely the spectrum of a free fermion of squared mass

$$
c_{F *}^{2}=\zeta_{\mathrm{ex}}^{2}
$$

in magnetic field. Translating to the $\nu$ variable we once again find the formula (4.56) but this time with

$$
s(\lambda)=\frac{\operatorname{sgn}\left(m_{F}\right)}{2}
$$

In other words excitations in the filled Landau levels behave like free particles that carry the 'unrenormalized' spin listed in (4.62) rather than the 'renormalized' spin listed in (4.57). We do not yet have a physical explanation for this striking result. We hope to return to this point in future work.

Note that it follows immediately from (4.61) together with the definition of $\chi_{n}$ that

$$
\chi_{n}=\frac{1}{2 \sqrt{\left(\zeta_{\mathrm{ex}}^{2}+2 b(n+1)\right)}} \quad \text { For } n \leq M
$$

Sewing the solutions together. Above we have found solutions for $\zeta_{ \pm}^{F}$ separately for $n \geq M+1$ and $n \leq M$. The solution in the two different ranges have been presented in terms of two independent integration constants $c_{F}$ and $c_{F *}$. Of course these integration constants are not really independent of each other; they are, in fact, easily related to each other as we now explain. Using the third lines in (4.41) and (4.44) and also the expressions for $\zeta_{R, I, M}$ and $\zeta_{R, I, M}$ listed in the first lines of those equations, and proceeding as in appendix $G$ it is possible to show that ${ }^{27}$

$$
\frac{1}{\chi_{M+1}^{2}}-\frac{1}{\chi_{M}^{2}}=-\left(2 \lambda_{F} b\right)^{2}\left(\chi_{M+1}^{2}\right)+8 b \pm 4 \lambda_{F} b
$$

Here ${ }^{\prime}+{ }^{\prime}$ is for positive $M$ band and ' $-{ }^{\prime}$ is for positive $M$ band. Substituting in the expressions for $\chi_{M+1}$ from (4.52) and the analogous expression for $\chi_{M}$ from (4.63), we find immediately from (4.64) that

$$
c_{F}^{2}=\zeta_{\mathrm{ex}}^{2} \pm \lambda_{F} b
$$

where we use the sign \pm depending on whether $\mu$ lies in the positive/negative $M^{\text {th }}$ band.

The gap equation for $\zeta_{\mathbf{e x}}$. We have now evaluated every quantity of interest in terms of a single unknown quantity $\zeta_{\mathrm{ex}}$. $\zeta_{\mathrm{ex}}$ may, in turn, be evaluated using the last 'junction' condition - which is equivalent to using the equation

$$
\frac{1}{\sqrt{b}} \Sigma_{R, I, 0}=-2 \lambda_{F} \sum_{k=M+1}^{\infty} \sqrt{b} \chi_{k}
$$

\footnotetext{
${ }^{27}$ The difference between positive and negative bands is in equation involving $\Sigma_{R,+, M+1}, \Sigma_{R,+, M+2}$.
} 
The r.h.s. of (4.66) is simplified using (4.54)

$$
\chi_{k}=\frac{\left(\zeta_{+}^{F}(k)-\zeta_{-}^{F}(k)\right)}{\left(\left(\zeta_{+}^{F}(k)\right)^{2}-\left(\zeta_{-}^{F}(k)\right)^{2}\right)}=\frac{\zeta_{+}^{F}(k)-\zeta_{-}^{F}(k)}{2 \lambda_{F} b}
$$

The 1.h.s. of (4.25) is simplified using

$$
\Sigma_{R, I, 0}=K_{R, I, 0}-m_{F}=\zeta_{\mathrm{ex}}-m_{F}
$$

We find

$$
m_{F}-\zeta_{\mathrm{ex}}=\sum_{k=M+1}^{\infty}\left(\left(\zeta_{\mathrm{ex}}^{2}+2 b(k+1)+\lambda_{F} b(1 \pm 1)\right)^{1 / 2}-\left(c_{F}^{2}+2 b(k+1)+\lambda_{F} b(-1 \pm 1)\right)^{1 / 2}\right)
$$

(where the sign \pm applies to $\mu$ in the positive/negative $M^{\text {th }}$ band.) More explicitly, when $\mu$ is in the $M^{t} h$ positive band

$$
m_{F}-\zeta_{\mathrm{ex}}=\sum_{k=M+1}^{\infty}\left(\zeta_{\mathrm{ex}}^{2}+2 b(k+1)+2 \lambda_{F} b\right)^{1 / 2}-\left(c_{F}^{2}+2 b(k+1)\right)^{1 / 2}
$$

while in the $M^{\text {th }}$ negative band

$$
m_{F}-\zeta_{\mathrm{ex}}=\sum_{k=M+1}^{\infty}\left(\zeta_{\mathrm{ex}}^{2}+2 b(k+1)\right)^{1 / 2}-\left(c_{F}^{2}+2 b(k+1)-2 \lambda_{F} b\right)^{1 / 2}
$$

\subsection{2 $\mu$ in the positive/negative exceptional band}

It turns out that the final results for the case that $\mu$ lies in the positive/negative exceptional band can be obtained from the results of the previous subsection by setting $M=-1$ in all final formulae. The derivation of these results closely parallel those presented in the previous subsection, and we will be brief in our presentation.

The gap equations in this case are listed in (4.42) and (4.43). Using these equations we find

$$
I_{n+1}-I_{n}=4 \quad(n \geq 0)
$$

It follows that

$$
\chi_{n}=\frac{1}{\sqrt{2}\left|\lambda_{F}\right| b}\left(\left(c_{F}^{2}+2 b(n+1)\right)-\left(\left(c_{F}^{2}+2 b(n+1)\right)^{2}-\left(\lambda_{F} b\right)^{2}\right)^{1 / 2}\right)^{1 / 2} \quad(n \geq 0)
$$

and

$$
\begin{aligned}
\zeta_{ \pm}^{F}(n) & =\frac{1}{2}\left( \pm 2 \lambda_{F} b \chi_{n}+\frac{1}{\chi_{n}}\right) \\
\Longrightarrow \zeta_{ \pm}^{F}(n) & =\left[c_{F}^{2}+2 b(n+1) \pm \lambda_{F} b\right]^{1 / 2} \quad(n \geq 0)
\end{aligned}
$$

Similar considerations as before gives

$$
c_{F}^{2}=\zeta_{\mathrm{ex}}^{2} \pm \lambda_{F} b
$$

Where ${ }^{\prime} \pm^{\prime}$ is taken for positive/negative bands. 
Once again our results can be rewritten in terms of the more physical variable $\nu$ labelling we find (in the appropriate range of $\nu$, i.e. the range that follows from $n \geq 0$ using (4.55)) that (4.56) and (4.57) apply. Once again we find agreement with (2.65) our naive guess for the spectrum of a theory of 'free particles of spin $s(\lambda)$ ' once we identify $c_{F}^{2}$ as the squared effective mass our excitation.

In this case there is no separate range (no analogue of the range $n \leq M$ ) Once again we find a gap equation for $\zeta_{\mathrm{ex}}$ using the gap equation

$$
\frac{1}{\sqrt{b}} \Sigma_{R, I, 0}=-2 \lambda_{F} \sum_{k=0}^{\infty} \sqrt{b} \chi_{k}
$$

Simplifying the r.h.s. and l.h.s. of (4.74) as before we obtain

$$
m_{F}-\zeta_{\mathrm{ex}}=\sum_{k=0}^{\infty}\left(\left(\zeta_{\mathrm{ex}}^{2}+2 b(k+1)+\lambda_{F} b(1 \pm 1)\right)^{1 / 2}-\left(c_{F}^{2}+2 b(k+1)+\lambda_{F} b(-1 \pm 1)\right)^{1 / 2}\right)
$$

(where the sign \pm applies to $\mu$ in the positive/negative exceptional band.) More explicitly, when $\mu$ is in the $M^{t h}$ positive exceptional band

$$
m_{F}-\zeta_{\mathrm{ex}}=\sum_{k=0}^{\infty}\left(\zeta_{\mathrm{ex}}^{2}+2 b(k+1)+2 \lambda_{F} b\right)^{1 / 2}-\left(c_{F}^{2}+2 b(k+1)\right)^{1 / 2}
$$

while in the $M^{t h}$ negative band

$$
m_{F}-\zeta_{\mathrm{ex}}=\sum_{k=0}^{\infty}\left(\zeta_{\mathrm{ex}}^{2}+2 b(k+1)\right)^{1 / 2}-\left(c_{F}^{2}+2 b(k+1)-2 \lambda_{F} b\right)^{1 / 2}
$$

\subsection{The $b \rightarrow 0$ limit and the regulated gap equation}

The gap equations (4.68), (4.69), (4.75) and (4.77) — which we need to solve to determine $\zeta_{\text {ex }}$ and hence $c_{F}$ are not well defined as they stand, as the summations on the r.h.s. of these equations are divergent. These gap equations need to be regulated and renormalized. In order to understand how this should be done in a physically sensible manner, it is useful to first consider the limit $b \rightarrow 0$. In this limit the gap equations presented in this paper reduce to the previously well studied gap equations for a massive fermion in the absence of a magnetic field.

Let us consider, for instance, the gap equation (4.68) which we reproduce here for convenience.

$$
m_{F}-\zeta_{\mathrm{ex}}=\sum_{k=M+1}^{\infty}\left(\zeta_{\mathrm{ex}}^{2}+2 b(k+1)+2 \lambda_{F} b\right)^{1 / 2}-\left(\zeta_{\mathrm{ex}}^{2}+2 b(k+1)\right)^{1 / 2}
$$

In the limit $b \rightarrow 0$ we get a significant contribution to the summation on the r.h.s. only for values of $k$ that scale like $k \sim \frac{1}{b}$. It is thus useful to work with the variable $w=b k$ and also to work with a value of $\mu$ that is held fixed as $b$ is taken to infinity (so that we work in the $M^{t h}$ positive band with $\mu=\sqrt{2 M b+\zeta_{\mathrm{ex}}^{2}}$. In other words we scale $M$ as

$$
M=\frac{\mu^{2}-\zeta_{\mathrm{ex}}^{2}}{2 b}
$$


with $\mu$ and $\zeta_{\text {ex }}$ held fixed. (4.78) simplifies to

$$
m_{F}-\zeta_{\mathrm{ex}}=\lambda_{F} \int_{\frac{\mu^{2}-\zeta_{\mathrm{ex}}^{2}}{2}}^{\infty} \frac{d w}{\sqrt{\zeta_{\mathrm{ex}}^{2}+2 w}}
$$

If we make the change of variables $2 w=p_{s}^{2}$ then (4.79) turns into

$$
\begin{aligned}
m_{F}-\zeta_{\mathrm{ex}} & =\lambda_{F} \int_{\sqrt{\mu^{2}-\zeta_{\mathrm{ex}}^{2}}}^{\infty} \frac{p_{s} d p_{s}}{\sqrt{\zeta_{\mathrm{ex}}^{2}+p_{s}^{2}}}=\lambda_{F} \int_{|\vec{p}|=\sqrt{\mu^{2}-\zeta_{\mathrm{ex}}^{2}}}^{\infty} \frac{d^{2} \vec{p}}{(2 \pi) \sqrt{\zeta_{\mathrm{ex}}^{2}+|\vec{p}|^{2}}} \\
& =\frac{\lambda_{F}}{2(2 \pi)^{2}} \int \frac{d^{3} p}{\zeta_{\mathrm{ex}}^{2}+p^{2}}
\end{aligned}
$$

where the integral in the final line of $(4.80)$ is taken over the contour $p_{3}=\omega=i \mu .^{28}$ The important point here is that the integrals on the r.h.s. of (4.80) are divergent; this is the continuum analogue of the divergence of the sum in (4.78). As explained in [4], this divergence may be regulated and renormalized away by continuing the integral over $p_{3}=\omega$ to an integral in $1-\epsilon$ dimensions. This regulation produces the integral

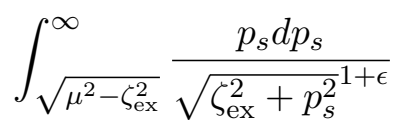

As explained in [4] this dimensionally regulated integral is easily evaluated; in-fact it evaluates to $|\mu| .{ }^{29}$

Dimensionally regulating the continuum integral (4.80) is equivalent to adding an appropriate counterterm to the field theory action that cancels the divergence in (4.79). In this paper we wish to study the same quantum field theory - the theory defined with the counterterm that affects the dimensional regulation of (4.79) - in the presence of a background magnetic field. We can accomplish this by manipulating (4.78) as follows

$$
\begin{aligned}
& m_{F}-\zeta_{\mathrm{ex}}=\sum_{k=M+1}^{\infty}\left(\zeta_{\mathrm{ex}}^{2}+2 b(k+1)+2 \lambda_{F} b\right)^{1 / 2}-\left(\zeta_{\mathrm{ex}}^{2}+2 b(k+1)\right)^{1 / 2} \\
& =\lim _{P \rightarrow \infty}\left[\sum_{k=M+1}^{P}\left(\left(\zeta_{\mathrm{ex}}^{2}+2 b(k+1)+2 \lambda_{F} b\right)^{1 / 2}-\left(\zeta_{\mathrm{ex}}^{2}+2 b(k+1)\right)^{1 / 2}\right)-\lambda_{F} \int_{\frac{\mu^{2}-\zeta_{\mathrm{ex}}^{2}}{2}}^{(P+1) b} \frac{d w}{\sqrt{\zeta_{\mathrm{ex}}^{2}+2 w}}\right]
\end{aligned}
$$

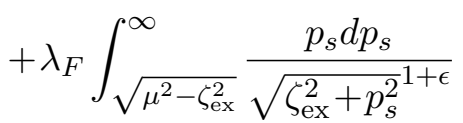

$$
\begin{aligned}
& =\lim _{P \rightarrow \infty}\left[\sum_{k=M+1}^{P}\left(\left(\zeta_{\mathrm{ex}}^{2}+2 b(k+1)+2 \lambda_{F} b\right)^{1 / 2}-\left(\zeta_{\mathrm{ex}}^{2}+2 b(k+1)\right)^{1 / 2}\right)-\lambda_{F} \int_{\frac{a^{2}}{2}}^{(P+1) b} \frac{d w}{\sqrt{\zeta_{\mathrm{ex}}^{2}+2 w}}\right] \\
& +\lambda_{F} \int_{a}^{\infty} \frac{p_{s} d p_{s}}{{\sqrt{\zeta_{\mathrm{ex}}^{2}+p_{s}^{2}}}^{1+\epsilon}}
\end{aligned}
$$

\footnotetext{
${ }^{28}$ The integral over $\omega=p_{3}$ on this contour produces the integral in the first line of $(4.80)$. Note that the integral over $\omega$ vanishes when $|\mu|^{2}>p_{s}^{2}+\zeta_{\mathrm{ex}}^{2}$.

${ }^{29}$ In this subsection we have assumed that $|\mu|>\left|\zeta_{\mathrm{ex}}\right|$; more generally the integral evaluates to $\max \left(|\mu|,\left|\zeta_{\mathrm{ex}}\right|\right)$.
} 
Several comments are in order. First note that the limits $P \rightarrow \infty$ in the second and fourth lines of (4.81) are well defined (i.e. finite); the divergence in the sums in these lines is cancelled by analogous divergence in the corresponding integrals. Next note that the integrals in the third and fifth lines of (4.81) are to be evaluated in a dimensionally regulated manner, i.e. are to be evaluated at a value of $\epsilon$ that is large enough to ensure the integral converges $(\epsilon>1)$; the result of the integral is then continued to $\epsilon=0$. It follows that the integrals on the third and fifth lines of (4.81) are also finite. ${ }^{30}$ Finally we have used the fact that the expression in the fourth and fifth lines of (4.81) does not depend on the variable $a$ (this can be verified by differentiating the expression w.r.t. $a$ ). This explains why the expression on the fourth and fifth lines of (4.81) equals the expression on the 2nd and 3rd lines of the same equation.

In this section we have, in particular, demonstrated that the gap equations presented in this paper reduce to the well known zero background field gap equations in the limit $b \rightarrow 0$, as expected in general grounds.

\subsection{Summary of final results for the fermion propagator}

The poles in the Euclidean propagator occur at the values

$$
\omega=-i\left(\zeta^{+}(n)-\mu\right) \text { and } \omega=-i\left(-\zeta^{n}(n)-\mu\right)
$$

- corresponding to Lorentzian quasi particle states at energies $\delta E=E-\mu$ at

$$
\delta E=\left(\zeta^{+}(n)-\mu\right) \text { and } \delta E=-\zeta^{-}(n)-\mu
$$

In this subsection we summarize our results for $\zeta_{ \pm}^{F}(n)$ for different cases.

\subsection{1 $\mu$ in the positive/negative $M$ band}

When $\mu$ is in the positive M band,

$$
\begin{aligned}
\zeta_{ \pm}^{F}(n) & =\left[\zeta_{\mathrm{ex}}^{2}+2 b(n+1)+\lambda_{F} b \pm \lambda_{F} b\right]^{1 / 2} & & (n \geq M+1) \\
\zeta_{ \pm}^{F}(n) & =\left[\zeta_{\mathrm{ex}}^{2}+2 b(n+1)\right]^{1 / 2} & & (n \leq M)
\end{aligned}
$$

When $\mu$ is in the negative $\mathrm{M}$ band,

$$
\begin{aligned}
& \zeta_{ \pm}^{F}(n)=\left[\zeta_{\mathrm{ex}}^{2}+2 b(n+1)-\lambda_{F} b \pm \lambda_{F} b\right]^{1 / 2} \quad(n \geq M+1) \\
& \zeta_{ \pm}^{F}(n)=\left[\zeta_{\mathrm{ex}}^{2}+2 b(n+1)\right]^{1 / 2} \quad(n \leq M)
\end{aligned}
$$

$\zeta_{\mathrm{ex}}$ is the solution to the equation

$$
\begin{aligned}
& m_{F}=\lambda_{F} \int_{a}^{\infty} \frac{p_{s} d p_{s}}{{\sqrt{\zeta_{\mathrm{ex}}^{2}+p_{s}^{2}}}^{1+\epsilon}}+\zeta_{\mathrm{ex}} \\
& +\lim _{P \rightarrow \infty}\left[\sum_{k=M+1}^{P}\left(\left(\zeta_{\mathrm{ex}}^{2}+2 b(k+1)+2 \lambda_{F} b\right)^{1 / 2}-\left(\zeta_{\mathrm{ex}}^{2}+2 b(k+1)\right)^{1 / 2}\right)-\lambda_{F} \int_{\frac{a^{2}}{2}}^{(P+1) b} \frac{d w}{\sqrt{\zeta_{\mathrm{ex}}^{2}+2 w}}\right]
\end{aligned}
$$

\footnotetext{
${ }^{30}$ These integrals can effectively be evaluated as follows. Working directly at $\epsilon=0$ we evaluate the integral as a difference between the indefinite integral evaluated at infinity and the same indefinite integral evaluated at the lower limit. The procedure of dimensional regularization effectively instructs us to discard the (divergent) term at $p_{s}=\infty$.
} 
for $\mu$ in the positive band while

$$
\begin{aligned}
& m_{F}=\lambda_{F} \int_{a}^{\infty} \frac{p_{s} d p_{s}}{{\sqrt{\zeta_{\mathrm{ex}}^{2}+p_{s}^{2}}}^{1+\epsilon}}+\zeta_{\mathrm{ex}} \\
& \lim _{P \rightarrow \infty}\left[\sum_{k=M+1}^{P}\left(\left(\zeta_{\mathrm{ex}}^{2}+2 b(k+1)\right)^{1 / 2}-\left(\zeta_{\mathrm{ex}}^{2}+2 b(k+1)-2 \lambda_{F} b\right)^{1 / 2}\right)-\lambda_{F} \int_{\frac{a^{2}}{2}}^{(P+1) b} \frac{d w}{\sqrt{\zeta_{\mathrm{ex}}^{2}+2 w}}\right]
\end{aligned}
$$

for $\mu$ in the negative $M$ band. In both (4.92) and (4.93), $a$ is any convenient quantity and the integrals on the r.h.s. of the first lines of these equations are the finite results for these integrals obtained in the dimensional regularization scheme. Explicitly

$$
\int_{a}^{\infty} \frac{p_{s} d p_{s}}{{\sqrt{\zeta_{\mathrm{ex}}^{2}+p_{s}^{2}}}^{1+\epsilon}}=-\sqrt{\zeta_{\mathrm{ex}}^{2}+a^{2}} \quad a \geq 0
$$

The full propagator is given by the expressions (4.23) and (4.19) where

$$
\Sigma_{R, I, 0}=\zeta_{\mathrm{ex}}-m_{F},
$$

$\Sigma_{R, I, n}$ may be evaluated from the first two lines of (4.41) and (4.43). ${ }^{31}$ and the expression for $\Sigma_{R,+, n}$ is listed subsequent lines of the same two equations. ${ }^{32}$

\subsection{2 $\mu$ in the positive/negative exceptional band}

When $\mu$ is in the positive $\mathrm{M}$ band,

$$
\zeta_{ \pm}^{F}(n)=\left[\zeta_{\mathrm{ex}}^{2}+2 b(n+1)+\lambda_{F} b \pm \lambda_{F} b\right]^{1 / 2} \quad(n \geq 0)
$$

When $\mu$ is in the negative $\mathrm{M}$ band,

$$
\zeta_{ \pm}^{F}(n)=\left[\zeta_{\mathrm{ex}}^{2}+2 b(n+1)-\lambda_{F} b \pm \lambda_{F} b\right]^{1 / 2} \quad(n \geq 0)
$$

$\zeta_{\mathrm{ex}}$ is the solution to the equation

$$
\begin{aligned}
& m_{F}=\lambda_{F} \int_{a}^{\infty} \frac{p_{s} d p_{s}}{{\sqrt{\zeta_{\mathrm{ex}}^{2}+p_{s}^{2}}}^{1+\epsilon}}+\zeta_{\mathrm{ex}} \\
& +\lim _{P \rightarrow \infty}\left[\sum_{k=0}^{P}\left(\left(\zeta_{\mathrm{ex}}^{2}+2 b(k+1)+2 \lambda_{F} b\right)^{1 / 2}-\left(\zeta_{\mathrm{ex}}^{2}+2 b(k+1)\right)^{1 / 2}\right)-\lambda_{F} \int_{\frac{a^{2}}{2}}^{(P+1) b} \frac{d w}{\sqrt{\zeta_{\mathrm{ex}}^{2}+2 w}}\right]
\end{aligned}
$$

for $\mu$ in the positive exceptional band while

$$
\begin{aligned}
& m_{F}=\lambda_{F} \int_{a}^{\infty} \frac{p_{s} d p_{s}}{{\sqrt{\zeta_{\mathrm{ex}}^{2}+p_{s}^{2}}}^{1+\epsilon}}+\zeta_{\mathrm{ex}} \\
& +\lim _{P \rightarrow \infty}\left[\sum_{k=0}^{P}\left(\left(\zeta_{\mathrm{ex}}^{2}+2 b(k+1)\right)^{1 / 2}-\left(\zeta_{\mathrm{ex}}^{2}+2 b(k+1)-2 \lambda_{F} b\right)^{1 / 2}\right)-\lambda_{F} \int_{\frac{a^{2}}{2}}^{(P+1) b} \frac{d w}{\sqrt{\zeta_{\mathrm{ex}}^{2}+2 w}}\right]
\end{aligned}
$$

for negative exceptional band. Once again $a$ is any convenient quantity and (4.88) applies.

\footnotetext{
${ }^{31}$ The infinite sums that occur in the second lines of these equations may be converted into finite sums by subtracting the expressions for $\Sigma_{R, I, n}$ from the expression for $\Sigma_{R, I, 0}$ whose explicit form we have presented in (4.89).

${ }^{32}$ The r.h.s. of the expressions that determine $\Sigma_{R,+, n}$ depends on $K_{R, I, n}$. This quantity is given in terms of $\Sigma_{R, I, n}$ - which we have already determined - in (4.17).
} 
Once again the full propagator is given by the expressions (4.23) and (4.19); the various quantities that occur in this expression are evaluated using (4.89), (4.41) and (4.43) as in the previous subsubsection.

\subsection{Gap equation in bosonic variables in exceptional bands with some choices of signs}

In this subsection we focus on the special case of $\mu$ in the positive/negative exceptional bands. We also assume that

$$
\begin{aligned}
& \zeta_{\mathrm{ex}} \lambda_{F}>0 \\
& \left.\lambda_{F}<0(\text { in }+ \text { ve exceptional band }) \text { and } \lambda_{F}>0 \text { (in - ve exceptional band }\right)
\end{aligned}
$$

We will see below that the conditions (4.94) lead to particularly simple gap equations in bosonic variables. We believe that the reason for this simplicity is that to ensure that the bosonic description is in an un Higgsed or uncondensed phase. With these assumptions we have two cases that we consider separately.

\subsection{1 $\zeta_{\mathrm{ex}}, \lambda_{F}$ all negative with $\mu$ in the positive exceptional band}

In this case the duality map takes the form

$$
\begin{aligned}
\lambda_{F} & =\lambda_{B}-1, \quad \lambda_{B}>0 \\
m_{F} & =-\lambda_{B} m_{B}^{\text {cri }},
\end{aligned}
$$

Substituting (4.95) into (4.92) turns the gap equation into

$$
\begin{aligned}
&-\lambda_{B} m_{B}^{\mathrm{cri}}=\left(\lambda_{B}-1\right) \int_{a}^{\infty} \frac{p_{s} d p_{s}}{\sqrt{\zeta_{\mathrm{ex}}^{2}+p_{s}^{2}}}{ }^{1+\epsilon} \\
&+\lim _{P \rightarrow \infty}\left[-\left|\zeta_{\mathrm{ex}}\right|+\sum_{k=0}^{P}\left(\left(\zeta_{\mathrm{ex}}^{2}+2 b k+2 \lambda_{B} b\right)^{1 / 2}-\left(\zeta_{\mathrm{ex}}^{2}+2 b(k+1)\right)^{1 / 2}\right)\right. \\
&\left.+\left(1-\lambda_{B}\right) \int_{\frac{a^{2}}{2}}^{(P+1) b} \frac{d w}{\sqrt{\zeta_{\mathrm{ex}}^{2}+2 w}}\right]
\end{aligned}
$$

Using

$$
\begin{aligned}
& -\left|\zeta_{\mathrm{ex}}\right|+\left(\sum_{k=0}^{P}\left[\left(\zeta_{\mathrm{ex}}^{2}+2 b k+2 \lambda_{B} b\right)^{1 / 2}-\left(\zeta_{\mathrm{ex}}^{2}+2 b(k+1)\right)^{1 / 2}\right]\right) \\
& =\left(\sum_{k=0}^{P}\left[\left(\zeta_{\mathrm{ex}}^{2}+2 b k+2 \lambda_{B} b\right)^{1 / 2}-\left(\zeta_{\mathrm{ex}}^{2}+2 b k\right)^{1 / 2}\right]\right)-\left(\zeta_{\mathrm{ex}}^{2}+2 b(P+1)\right)^{1 / 2}
\end{aligned}
$$

we can rewrite (4.98) as

$$
\begin{aligned}
&-\lambda_{B} m_{B}^{\mathrm{cri}}=\left(\lambda_{B}-1\right) \int_{a}^{\infty} \frac{p_{s} d p_{s}}{\sqrt{\zeta_{\mathrm{ex}}^{2}+p_{s}^{2}}} 1+\epsilon \\
&+\lim _{P \rightarrow \infty}\left[\sum_{k=0}^{P}\left[\left(\zeta_{\mathrm{ex}}^{2}+2 b k+2 \lambda_{B} b\right)^{1 / 2}-\left(\zeta_{\mathrm{ex}}^{2}+2 b k\right)^{1 / 2}\right]\right. \\
&\left.-\left(\zeta_{\mathrm{ex}}^{2}+2 b(P+1)\right)^{1 / 2}+\left(1-\lambda_{B}\right) \int_{\frac{a^{2}}{2}}^{(P+1) b} \frac{d w}{\sqrt{\zeta_{\mathrm{ex}}^{2}+2 w}}\right]
\end{aligned}
$$


Using

$$
\int_{a}^{(P+1) b} \frac{d w}{\sqrt{\zeta_{\mathrm{ex}}^{2}+2 w}}=\left(\zeta_{\mathrm{ex}}^{2}+2 b(P+1)\right)^{1 / 2}-\left(\zeta_{\mathrm{ex}}^{2}+a^{2}\right)^{1 / 2}
$$

and (4.88) we see that (4.98) can be re-expressed as

$$
\begin{aligned}
-\lambda_{B} m_{B}^{\mathrm{cri}}= & \lambda_{B} \int_{a}^{\infty} \frac{p_{s} d p_{s}}{{\sqrt{\zeta_{\mathrm{ex}}^{2}+p_{s}^{2}}}^{1+\epsilon}} \\
& +\lim _{P \rightarrow \infty}\left[\sum_{k=0}^{P}\left[\left(\zeta_{\mathrm{ex}}^{2}+2 b k+2 \lambda_{B} b\right)^{1 / 2}-\left(\zeta_{\mathrm{ex}}^{2}+2 b k\right)^{1 / 2}\right]-\lambda_{B} \int_{\frac{a^{2}}{2}}^{(P+1) b} \frac{d w}{\sqrt{\zeta_{\mathrm{ex}}^{2}+2 w}}\right]
\end{aligned}
$$

Note that every term in (4.103) is of order $\lambda_{B}$ at small $\lambda_{B}$. It follows that (4.103) has a smooth $\lambda_{B} \rightarrow 0$ limit.

Finally we note that (4.103) has an aestheically unpleasing feature. The summation in the second line of this equation is a discretised version of an integral with upper limit $w=P b$. However the 'subtraction integral' in the second line of (4.103) has an upper limit $(P+1) b$. Note however that

$$
\int_{\frac{a^{2}}{2}}^{(P+r) b} \frac{d w}{\sqrt{\zeta_{\mathrm{ex}}^{2}+2 w}}=\int_{\frac{a^{2}}{2}}^{(P+s) b} \frac{d w}{\sqrt{\zeta_{\mathrm{ex}}^{2}+2 w}}+\mathcal{O}\left(\frac{1}{\sqrt{P}}\right)
$$

where $r$ and $s$ are any numbers that are held fixed in the limit $P \rightarrow 0$. It follows that (4.103) is equivalent to the aesthetically more pleasing gap equation

$$
\begin{aligned}
-\lambda_{B} m_{B}^{\mathrm{cri}}= & \lambda_{B} \int_{a}^{\infty} \frac{p_{s} d p_{s}}{{\sqrt{\zeta_{\mathrm{ex}}^{2}+p_{s}^{2}}}^{1+\epsilon}} \\
& +\lim _{P \rightarrow \infty}\left[\sum_{k=0}^{P}\left[\left(\zeta_{\mathrm{ex}}^{2}+2 b k+2 \lambda_{B} b\right)^{1 / 2}-\left(\zeta_{\mathrm{ex}}^{2}+2 b k\right)^{1 / 2}\right]-\lambda_{B} \int_{\frac{a^{2}}{2}}^{P b} \frac{d w}{\sqrt{\zeta_{\mathrm{ex}}^{2}+2 w}}\right]
\end{aligned}
$$

\subsection{2 $\zeta_{\mathrm{ex}}, \lambda_{F}$ all positive with $\mu$ in the negative exceptional band}

$$
\begin{aligned}
-\lambda_{B} m_{B}^{\mathrm{cri}}= & \lambda_{B} \int_{a}^{\infty} \frac{p_{s} d p_{s}}{{\sqrt{\zeta_{\mathrm{ex}}^{2}+p_{s}^{2}}}^{1+\epsilon}} \\
& +\lim _{P \rightarrow \infty}\left[\sum_{k=0}^{P}\left[\left(\zeta_{\mathrm{ex}}^{2}+2 b k+2 \lambda_{B} b\right)^{1 / 2}-\left(\zeta_{\mathrm{ex}}^{2}+2 b k\right)^{1 / 2}\right]-\lambda_{B} \int_{\frac{a^{2}}{2}}^{(P+1) b} \frac{d w}{\sqrt{\zeta_{\mathrm{ex}}^{2}+2 w}}\right]
\end{aligned}
$$

In this case the duality map takes the form

$$
\begin{aligned}
\lambda_{F} & =1+\lambda_{B} \quad \lambda_{B}<0 \\
m_{F} & =-\lambda_{B} m_{B}^{\text {cri }},
\end{aligned}
$$

Substituting (4.104) into (4.93) we obtain

$$
\begin{aligned}
& -\lambda_{B} m_{B}^{\mathrm{cri}}=\left(1+\lambda_{B}\right) \int_{a}^{\infty} \frac{p_{s} d p_{s}}{{\sqrt{\zeta_{\mathrm{ex}}^{2}+p_{s}^{2}}}^{1+\epsilon}}+\zeta_{\mathrm{ex}} \\
& +\lim _{P \rightarrow \infty}\left[\sum_{k=0}^{P}\left(\left(\zeta_{\mathrm{ex}}^{2}+2 b(k+1)\right)^{1 / 2}-\left(\zeta_{\mathrm{ex}}^{2}+2 b k-2 \lambda_{B} b\right)^{1 / 2}\right)-\left(1+\lambda_{B}\right) \int_{\frac{a^{2}}{2}}^{(P+1) b} \frac{d w}{\sqrt{\zeta_{\mathrm{ex}}^{2}+2 w}}\right]
\end{aligned}
$$


Rearranging terms as in the previous subsubsection we find

$$
\begin{aligned}
-\lambda_{B} m_{B}^{\mathrm{cri}}=\left(1+\lambda_{B}\right) & \int_{a}^{\infty} \frac{p_{s} d p_{s}}{{\sqrt{\zeta_{\mathrm{ex}}^{2}+p_{s}^{2}}}^{1+\epsilon}} \\
+\lim _{P \rightarrow \infty}[ & \sum_{k=0}^{P}\left(\left(\zeta_{\mathrm{ex}}^{2}+2 b k\right)^{1 / 2}-\left(\zeta_{\mathrm{ex}}^{2}+2 b k-2 \lambda_{B} b\right)^{1 / 2}\right) \\
& \left.+\left(\zeta_{\mathrm{ex}}^{2}+2 b(P+1)\right)^{1 / 2}-\left(1+\lambda_{B}\right) \int_{\frac{a^{2}}{2}}^{(P+1) b} \frac{d w}{\sqrt{\zeta_{\mathrm{ex}}^{2}+2 w}}\right]
\end{aligned}
$$

As in the previous subsubsection, this equation can be further simplified to

$$
\begin{aligned}
-\lambda_{B} m_{B}^{\mathrm{cri}}= & \lambda_{B} \int_{a}^{\infty} \frac{p_{s} d p_{s}}{\sqrt{\zeta_{\mathrm{ex}}^{2}+p_{s}^{2}} 1+\epsilon} \\
& +\lim _{P \rightarrow \infty}\left[\sum_{k=0}^{P}\left(\left(\zeta_{\mathrm{ex}}^{2}+2 b k\right)^{1 / 2}-\left(\zeta_{\mathrm{ex}}^{2}+2 b k-2 \lambda_{B} b\right)^{1 / 2}\right)-\lambda_{B} \int_{\frac{a^{2}}{2}}^{(P+1) b} \frac{d w}{\sqrt{\zeta_{\mathrm{ex}}^{2}+2 w}}\right]
\end{aligned}
$$

As in the previous subsection, every term in (4.107) is of order $\lambda_{B}$ at small $\lambda_{B}$ and so the equation (4.107) has a smooth $\lambda_{B} \rightarrow 0$ limit. As in the previous subsubsection, we may use (4.101) to recast this equation in the aesthetically more pleasing form

$$
\begin{aligned}
-\lambda_{B} m_{B}^{\mathrm{cri}}= & \lambda_{B} \int_{a}^{\infty} \frac{p_{s} d p_{s}}{{\sqrt{\zeta_{\mathrm{ex}}^{2}+p_{s}^{2}}}^{1+\epsilon}} \\
& +\lim _{P \rightarrow \infty}\left[\sum_{k=0}^{P}\left(\left(\zeta_{\mathrm{ex}}^{2}+2 b k\right)^{1 / 2}-\left(\zeta_{\mathrm{ex}}^{2}+2 b k-2 \lambda_{B} b\right)^{1 / 2}\right)-\lambda_{B} \int_{\frac{a^{2}}{2}}^{P b} \frac{d w}{\sqrt{\zeta_{\mathrm{ex}}^{2}+2 w}}\right]
\end{aligned}
$$

\subsection{The gap equations in bosonic variables in the generic case}

\subsection{1 $\mu$ in positive $M$ band, $\lambda_{F}$ negative}

In this case (4.95) applies. Using manipulations similar to those in the previous subsection, the gap equations in this case can be recast as

$$
\begin{aligned}
-\lambda_{B} m_{B}^{\mathrm{cri}}= & \lambda_{B} \int_{a}^{\infty} \frac{p_{s} d p_{s}}{\sqrt{\zeta_{\mathrm{ex}}^{2}+p_{s}^{2}}}{ }^{1+\epsilon} \\
& +\lim _{P \rightarrow \infty}\left[\sum_{k=M+1}^{P}\left[\left(\zeta_{\mathrm{ex}}^{2}+2 b k+2 \lambda_{B} b\right)^{1 / 2}-\left(\zeta_{\mathrm{ex}}^{2}+2 b k\right)^{1 / 2}\right]-\lambda_{B} \int_{\frac{a^{2}}{2}}^{(P+1) b} \frac{d w}{\sqrt{\zeta_{\mathrm{ex}}^{2}+2 w}}\right] \\
& +\zeta_{\mathrm{ex}}+\left(\zeta_{\mathrm{ex}}^{2}+2 b(M+1)\right)^{1 / 2}
\end{aligned}
$$

As in the previous subsection we can use (4.101) to rewrite (4.109) in the equivalent but aesthetically more pleasing form

$$
\begin{aligned}
-\lambda_{B} m_{B}^{\mathrm{cri}}= & \lambda_{B} \int_{a}^{\infty} \frac{p_{s} d p_{s}}{{\sqrt{\zeta_{\mathrm{ex}}^{2}+p_{s}^{2}}}^{1+\epsilon}} \\
& +\lim _{P \rightarrow \infty}\left[\sum_{k=M+1}^{P}\left[\left(\zeta_{\mathrm{ex}}^{2}+2 b k+2 \lambda_{B} b\right)^{1 / 2}-\left(\zeta_{\mathrm{ex}}^{2}+2 b k\right)^{1 / 2}\right]-\lambda_{B} \int_{\frac{a^{2}}{2}}^{P b} \frac{d w}{\sqrt{\zeta_{\mathrm{ex}}^{2}+2 w}}\right] \\
& +\zeta_{\mathrm{ex}}+\left(\zeta_{\mathrm{ex}}^{2}+2 b(M+1)\right)^{1 / 2}
\end{aligned}
$$


This result (4.109) at $M=-1$ applies to the positive exceptional band. In this case the last line of (4.109) reduces to

$$
+\zeta_{\mathrm{ex}}+\left|\zeta_{\mathrm{ex}}\right|
$$

and vanishes when $\zeta_{\text {ex }}$ is negative in agreement with (4.103). In every other case (i.e. if $M>-1$ or if $M=-1$ and $\zeta_{\mathrm{ex}}$ is positive) the third line of (4.109) is nonzero, and is, moreover, of order unity (rather than order $\lambda_{B}$ ) in the limit $\lambda_{B} \rightarrow 0$. It follows that in these cases the limit $\lambda_{B} \rightarrow 0$ of (4.109) is not smooth.

\subsection{2 $\mu$ in negative $M$ band, $\lambda_{F}$ positive}

In this case (4.104) applies. Using manipulations similar to those in the previous subsection, the gap equations can be shown to take the form

$$
\begin{aligned}
-\lambda_{B} m_{B}^{\mathrm{cri}}= & \lambda_{B} \int_{a}^{\infty} \frac{p_{s} d p_{s}}{\sqrt{\zeta_{\mathrm{ex}}^{2}+p_{s}^{2}}}{ }^{1+\epsilon} \\
& +\lim _{P \rightarrow \infty}\left[\sum_{k=M+1}^{P}\left[\left(\zeta_{\mathrm{ex}}^{2}+2 b k\right)^{1 / 2}-\left(\zeta_{\mathrm{ex}}^{2}+2 b k-2 \lambda_{B} b\right)^{1 / 2}\right]-\lambda_{B} \int_{\frac{a^{2}}{2}}^{(P+1) b} \frac{d w}{\sqrt{\zeta_{\mathrm{ex}}^{2}+2 w}}\right] \\
& +\zeta_{\mathrm{ex}}-\left(\zeta_{\mathrm{ex}}^{2}+2 b(M+1)\right)^{1 / 2}
\end{aligned}
$$

Again the result (4.111) can be rewritten (using (4.101)) as

$$
\begin{aligned}
-\lambda_{B} m_{B}^{\mathrm{cri}}= & \lambda_{B} \int_{a}^{\infty} \frac{p_{s} d p_{s}}{{\sqrt{\zeta_{\mathrm{ex}}^{2}+p_{s}^{2}}}^{1+\epsilon}} \\
& +\lim _{P \rightarrow \infty}\left[\sum_{k=M+1}^{P}\left[\left(\zeta_{\mathrm{ex}}^{2}+2 b k\right)^{1 / 2}-\left(\zeta_{\mathrm{ex}}^{2}+2 b k-2 \lambda_{B} b\right)^{1 / 2}\right]-\lambda_{B} \int_{\frac{a^{2}}{2}}^{P b} \frac{d w}{\sqrt{\zeta_{\mathrm{ex}}^{2}+2 w}}\right] \\
& +\zeta_{\mathrm{ex}}-\left(\zeta_{\mathrm{ex}}^{2}+2 b(M+1)\right)^{1 / 2}
\end{aligned}
$$

This result (4.111) at $M=-1$ applies to the negative exceptional band. In this case the last line of (4.111) reduces to

$$
+\zeta_{\mathrm{ex}}-\left|\zeta_{\mathrm{ex}}\right|
$$

and vanishes when $\zeta_{\mathrm{ex}}$ is positive in agreement with (4.107). In every other case (i.e. if $M>-1$ or if $M=-1$ and $\zeta_{\mathrm{ex}}$ is negative) the third line of (4.111) is nonzero, and is, moreover, of order unity (rather than order $\lambda_{B}$ ); it follows that the equation (4.111) does not have a smooth $\lambda_{b} \rightarrow 0$ limit. 


\subsection{3 $\mu$ in positive $M$ band, $\lambda_{F}$ positive}

In this case the duality map takes the form (4.104). Re-expressing (4.92) in dual variables and using

$$
\begin{aligned}
& \sum_{k=M+1}^{P}\left(\left(\zeta_{\mathrm{ex}}^{2}+2 b(k+2)+2 \lambda_{B} b\right)^{1 / 2}-\left(\zeta_{\mathrm{ex}}^{2}+2 b(k+1)\right)^{1 / 2}\right) \\
&-\left(\lambda_{B}+1\right) \int_{\frac{a^{2}}{2}}^{(P+1) b} \frac{d w}{\sqrt{\zeta_{\mathrm{ex}}^{2}+2 w}}+\left(\lambda_{B}+1\right) \int_{a}^{\infty} \frac{p_{s} d p_{s}}{{\sqrt{\zeta_{\mathrm{ex}}^{2}+p_{s}^{2}}}^{1+\epsilon}} \\
&= {\left[\sum_{k=M+1}^{P}\left(\left(\zeta_{\mathrm{ex}}^{2}+2 b(k+2)+2 \lambda_{B} b\right)^{1 / 2}-\left(\zeta_{\mathrm{ex}}^{2}+2 b(k+2)\right)^{1 / 2}\right)-\lambda_{B} \int_{\frac{a^{2}}{2}}^{(P+1) b} \frac{d w}{{\sqrt{\zeta_{\mathrm{ex}}^{2}+2 w}}^{2}}\right] } \\
&+\left(\zeta_{\mathrm{ex}}^{2}+2 b(P+2)\right)^{1 / 2}-\left(\zeta_{\mathrm{ex}}^{2}+2 b(M+2)\right)^{1 / 2} \\
&-\left(\zeta_{\mathrm{ex}}^{2}+2 b(P+1)\right)^{1 / 2}+\lambda_{B} \int_{a}^{\infty} \frac{p_{s} d p_{s}}{{\sqrt{\zeta_{\mathrm{ex}}^{2}+p_{s}^{2}}}^{1+\epsilon}}
\end{aligned}
$$

and (4.101) we find

$$
\begin{aligned}
-\lambda_{B} m_{B}^{\mathrm{cri}}= & \lambda_{B} \int_{a}^{\infty} \frac{p_{s} d p_{s}}{{\sqrt{\zeta_{\mathrm{ex}}^{2}+p_{s}^{2}}}^{1+\epsilon}} \\
& +\lim _{P \rightarrow \infty}\left[\sum_{k=M+3}^{P+2}\left[\left(\zeta_{\mathrm{ex}}^{2}+2 b k+2 \lambda_{B} b\right)^{1 / 2}-\left(\zeta_{\mathrm{ex}}^{2}+2 b k\right)^{1 / 2}\right]-\lambda_{B} \int_{\frac{a^{2}}{2}}^{(P+1) b} \frac{d w}{\sqrt{\zeta_{\mathrm{ex}}^{2}+2 w}}\right] \\
& +\zeta_{\mathrm{ex}}-\left(\zeta_{\mathrm{ex}}^{2}+2 b(M+2)\right)^{1 / 2} \\
& +\left(\zeta_{\mathrm{ex}}^{2}+2 b(P+2)\right)^{1 / 2}-\left(\zeta_{\mathrm{ex}}^{2}+2 b(P+1)\right)^{1 / 2}
\end{aligned}
$$

\subsection{4 $\mu$ in negative $M$ band, $\lambda_{F}$ negative}

In this case the duality map takes the form (4.95). Reexpressing (4.93) in dual variables and using

$$
\begin{aligned}
& \sum_{k=M+1}^{P}\left(\left(\zeta_{\mathrm{ex}}^{2}+2 b(k+1)\right)^{1 / 2}-\left(\zeta_{\mathrm{ex}}^{2}+2 b(k+2)-2 \lambda_{B} b\right)^{1 / 2}\right) \\
& -\left(\lambda_{B}-1\right) \int_{\frac{a^{2}}{2}}^{(P+1) b} \frac{d w}{\sqrt{\zeta_{\mathrm{ex}}^{2}+2 w}}+\left(\lambda_{B}-1\right) \int_{a}^{\infty} \frac{p_{s} d p_{s}}{{\sqrt{\zeta_{\mathrm{ex}}^{2}+p_{s}^{2}}}^{1+\epsilon}} \\
= & \sum_{k=M+1}^{P}\left[\left(\zeta_{\mathrm{ex}}^{2}+2 b(k+2)\right)^{1 / 2}-\left(\zeta_{\mathrm{ex}}^{2}+2 b(k+2)-2 \lambda_{B} b\right)^{1 / 2}-\lambda_{B} \int_{\frac{a^{2}}{2}}^{(P+1) b} \frac{d w}{\sqrt{\zeta_{\mathrm{ex}}^{2}+2 w}}\right] \\
& +\left(\zeta_{\mathrm{ex}}^{2}+2 b(M+2)\right)^{1 / 2}-\left(\zeta_{\mathrm{ex}}^{2}+2 b(P+2)\right)^{1 / 2} \\
& +\lambda_{B} \int_{a}^{\infty} \frac{p_{s} d p_{s}}{{\sqrt{\zeta_{\mathrm{ex}}^{2}+p_{s}^{2}}}^{1+\epsilon}}+\left(\zeta_{\mathrm{ex}}^{2}+2 b(P+1)\right)^{1 / 2}
\end{aligned}
$$


along with (4.101), the gap equation becomes

$$
\begin{aligned}
-\lambda_{B} m_{B}^{\mathrm{cri}}= & \lambda_{B} \int_{a}^{\infty} \frac{p_{s} d p_{s}}{{\sqrt{\zeta_{\mathrm{ex}}^{2}+p_{s}^{2}}}^{1+\epsilon}} \\
& +\lim _{P \rightarrow \infty}\left[\sum_{k=M+3}^{P+2}\left[\left(\zeta_{\mathrm{ex}}^{2}+2 b k\right)^{1 / 2}-\left(\zeta_{\mathrm{ex}}^{2}+2 b k-2 \lambda_{B} b\right)^{1 / 2}\right]-\lambda_{B} \int_{\frac{a^{2}}{2}}^{(P+1) b} \frac{d w}{\sqrt{\zeta_{\mathrm{ex}}^{2}+2 w}}\right] \\
& +\zeta_{\mathrm{ex}}+\left(\zeta_{\mathrm{ex}}^{2}+2 b(M+2)\right)^{1 / 2} \\
& -\left(\zeta_{\mathrm{ex}}^{2}+2 b(P+2)\right)^{1 / 2}
\end{aligned}
$$

\subsection{Solving for $\zeta_{\mathrm{ex}}$}

Consider, for instance, the positive M band gap equation (4.92) which we reproduce here for convenience

$$
\begin{aligned}
& m_{F}=\lambda_{F} \int_{a}^{\infty} \frac{p_{s} d p_{s}}{{\sqrt{\zeta_{\mathrm{ex}}^{2}+p_{s}^{2}}}^{1+\epsilon}}+\zeta_{\mathrm{ex}} \\
& +\lim _{P \rightarrow \infty}\left[\sum_{k=M+1}^{P}\left(\left(\zeta_{\mathrm{ex}}^{2}+2 b(k+1)+2 \lambda_{F} b\right)^{1 / 2}-\left(\zeta_{\mathrm{ex}}^{2}+2 b(k+1)\right)^{1 / 2}\right)-\lambda_{F} \int_{\frac{a^{2}}{2}}^{(P+1) b} \frac{d w}{\sqrt{\zeta_{\mathrm{ex}}^{2}+2 w}}\right]
\end{aligned}
$$

Making the choice $a=0$ we evaluate the first integral (4.117) to obtain the simplified equation

$$
\begin{aligned}
m_{F}= & \zeta_{\mathrm{ex}}-\lambda_{F}\left|\zeta_{\mathrm{ex}}\right|-\sum_{k=0}^{M}\left(\left(\zeta_{\mathrm{ex}}^{2}+2 b(k+1)+2 \lambda_{F} b\right)^{1 / 2}-\left(\zeta_{\mathrm{ex}}^{2}+2 b(k+1)\right)^{1 / 2}\right) \\
& +\lim _{P \rightarrow \infty}\left[\sum_{k=0}^{P}\left(\left(\zeta_{\mathrm{ex}}^{2}+2 b(k+1)+2 \lambda_{F} b\right)^{1 / 2}-\left(\zeta_{\mathrm{ex}}^{2}+2 b(k+1)\right)^{1 / 2}\right)-\lambda_{F} \int_{0}^{(P+1) b} \frac{d w}{\sqrt{\zeta_{\mathrm{ex}}^{2}+2 w}}\right] \\
\equiv & f\left(\zeta_{\mathrm{ex}}, \lambda_{F}\right)
\end{aligned}
$$

\subsubsection{Existence of solutions}

The second and third lines of (4.118) are both of order $\mathcal{O}\left(1 /\left|\zeta_{\mathrm{ex}}\right|\right)$ at large $\left|\zeta_{\mathrm{ex}}\right| \cdot{ }^{33}$ It follows that the r.h.s. of (4.118) behaves like $\left(1-\lambda_{F}\right)\left|\zeta_{\mathrm{ex}}\right|$ as $\zeta_{\mathrm{ex}} \rightarrow \infty$, but behaves like $-\left(1+\lambda_{F}\right)\left|\zeta_{\mathrm{ex}}\right|$ as $\zeta_{\mathrm{ex}} \rightarrow-\infty$. As the r.h.s. of (4.118) is a continuous function of $\zeta_{\mathrm{ex}}$, it follows that it takes every real value as $\zeta_{\text {ex }}$ varies from $-\infty$ to $\infty$, except possibly the extreme value $\lambda_{F}= \pm 1 .^{34}$ As a consequence (4.118) has at least one real solution (for the variable $\left.\zeta_{\mathrm{ex}}\right)$ for every choice of $m_{F}$ provided $\left|\lambda_{F}\right|<1$. It is easily verified that this conclusion continues to hold for negative bands and also for exceptional bands.

\subsubsection{Uniqueness of solutions}

Consider the equation (4.118) at any fixed $\lambda_{F}$. We have already seen that the r.h.s. of this equation increases from $-\infty$ to $\infty$ as $\zeta_{\text {ex }}$ varies from $-\infty$ to $\infty$. If this increase occurs

\footnotetext{
${ }^{33}$ Here we are measuring all dimensionful qualities in the units of magnetic field.

${ }^{34}$ Recall that $\left|\lambda_{F}\right| \leq 1$.
} 


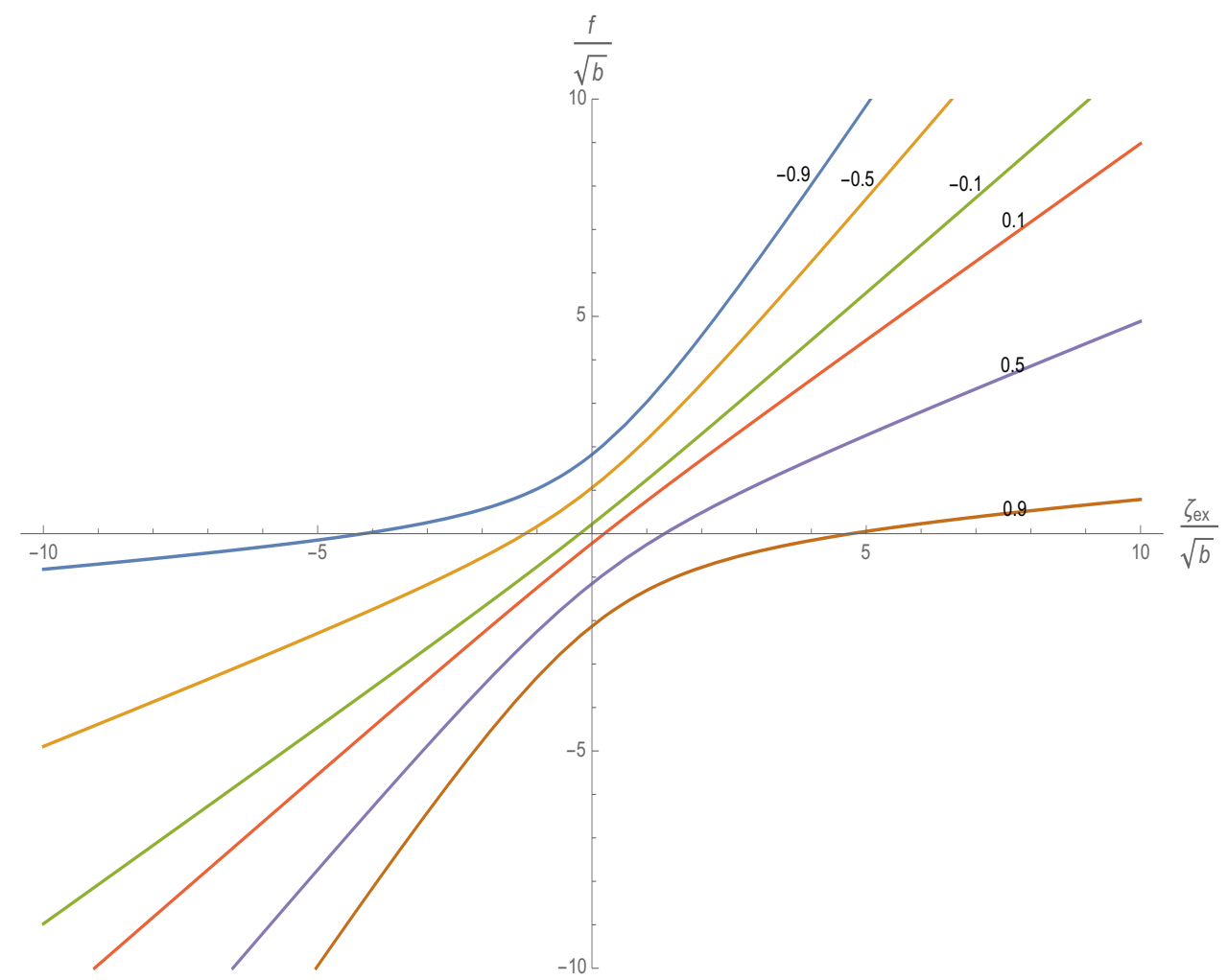

Figure 1. Here we have plotted $f$ defined in (4.118) for different values of $\lambda_{F}$ that are mentioned by the side of each curve. This qualitative behaviour is valid for both positive and negative bands including exceptional ones.

monotonically, it follows that (4.118) has a unique solution for every value of $m_{F}$. If, on the other hand, the r.h.s. of (4.118) is not a monotonic function of $\zeta_{\text {ex }}$ then there exists a range of $m_{F}$ over which there exist three or more solutions to the equation (4.118).

When $\lambda_{F}$ is small, the second and third lines of (4.118) are both of order $\lambda_{F}$. In this limit the r.h.s. of (4.118) is approximately given by $\zeta_{\mathrm{ex}}$ and so is monotonic. It follows that the solutions of (4.118) are unique at small enough $\lambda_{F}$. Below we will also argue that the solutions of (4.118) are also unique when $\lambda_{F}$ is in the vicinity of \pm 1 We do not know whether this property (monotonicity of the r.h.s. of (4.118) and consequent uniqueness of the solutions to the gap equation) persists at arbitrary values of $\lambda_{F}$. Preliminary numerical experiments suggest that the uniqueness of solutions may persist for all values of $\lambda_{F}$ and all values of $M$ (see figure 1). This question deserves a more careful numerical study which we defer to future work. Again all the conclusions of this subsection apply also to negative and exceptional bands.

\subsubsection{Perturbation theory in small $\lambda_{F}$}

We have argued above that (4.118) has a unique solution (at least) at small enough $\lambda_{F}$. In a perturbative expansion in $\lambda_{F}$ this solution takes the form

$$
\zeta_{\mathrm{ex},+, M}=m_{F}+\sum_{n=1}^{\infty} \lambda_{F}^{n} \zeta_{\mathrm{ex},+, M}^{n}
$$


It is not difficult to obtain explicit expressions for the coefficients $\zeta_{\mathrm{ex},+, M}^{n}$ at small values of $n$. The expressions are less than completely illuminating as they are given in terms of (completely explicit) infinite summations. For this reason we present explicit formulae only for $\zeta_{\mathrm{ex},+, M}^{1}$ below

$$
\begin{aligned}
\zeta_{\mathrm{ex},+, M}^{1}= & \left|m_{F}\right|+\sum_{n=0}^{M} \frac{1}{\sqrt{m_{F}^{2}+2 b(n+1)}} \\
& -\lim _{P \rightarrow \infty}\left[\sum_{n=0}^{P} \frac{1}{\sqrt{m_{F}^{2}+2 b(n+1)}}-\int_{0}^{b(P+1)} d w \frac{1}{\sqrt{m_{F}^{2}+2 b n}}\right]
\end{aligned}
$$

Note that the complicated part of the solution (the second line of (4.120)) is independent of $M$. This fact allows us to reach a simple explicit conclusion about the energy width of Landau levels, as we now explain.

Let us first assume that physical interpretation of $\mu$ lying in the $M^{\text {th }}$ positive energy gap is that all Landau levels with $n \leq M$ are completely filled. When this is the case, the formula for the energy of the Landau level at $n=M$ is given by $E_{+, M}=\zeta_{+}^{F}(M)$ where

$$
\left(E_{+, M}^{\mathrm{up}}\right)^{2}=\zeta_{\mathrm{ex}, M}^{2}+2 b(M+1)
$$

The energy $E_{+, M}^{\mathrm{up}}$ reported above may physically be thought of as the energy of the last 'electron' whose addition completely filled up the Landau Level with $n=M$.

On the other hand when $\mu$ lies in the $M-1$ positive level, the energy of the Landau level with $n=M$ is given by

$$
\left(E_{+, M}^{\text {low }}\right)^{2}=\zeta_{\mathrm{ex}, M-1}^{2}+2 b(M+1)+2 \lambda_{F} b
$$

Physically, $E_{+, M}^{\text {low }}$ is the energy of the first 'electron' added to the Landau level with $n=M$.

Notice that

$$
\left(E_{+, M}^{\mathrm{up}}\right)^{2}-\left(E_{+, M}^{\mathrm{low}}\right)^{2}=\zeta_{\mathrm{ex}, M}^{2}-\zeta_{\mathrm{ex}, M-1}^{2}-2 \lambda_{F} b
$$

It follows from (4.120) that

$$
\Delta_{+}(M)=\left(E_{+, M}^{\text {up }}\right)^{2}-\left(E_{+, M}^{\text {low }}\right)^{2}=-2 \lambda_{F} b\left(1-\frac{m_{F}}{\sqrt{m_{F}^{2}+2 b(M+1)}}\right)+\mathcal{O}\left(\lambda_{F}^{2}\right)
$$

Similar considerations for negative $M$ band shows that ${ }^{35}$

$$
\begin{aligned}
\zeta_{\mathrm{ex},-, M}^{1}= & \left|m_{F}\right|+\sum_{n=0}^{M} \frac{1}{\sqrt{m_{F}^{2}+2 b(n+1)}} \\
& -\lim _{P \rightarrow \infty}\left[\sum_{n=0}^{P} \frac{1}{\sqrt{m_{F}^{2}+2 b(n+1)}}-\int_{0}^{b(P+1)} d w \frac{1}{\sqrt{m_{F}^{2}+2 b n}}\right] \\
\Delta_{-}(M)= & \left(E_{-, M}^{\mathrm{up}}\right)^{2}-\left(E_{-, M}^{\mathrm{low}}\right)^{2}=-2 \lambda_{F} b\left(1+\frac{m_{F}}{\sqrt{m_{F}^{2}+2 b(M+1)}}\right)+\mathcal{O}\left(\lambda_{F}^{2}\right)
\end{aligned}
$$

${ }^{35}$ Note that in this case $E_{-, M}=-\zeta_{-}^{F}(M)$

$$
\left(E_{-, M}^{\text {up }}\right)^{2}=\zeta_{\mathrm{ex}, M-1}^{2}+2 b(M+1)-2 \lambda_{F} b, \quad\left(E_{-, M}^{\mathrm{low}}\right)^{2}=\zeta_{\mathrm{ex}, M}^{2}+2 b(M+1) .
$$


On the other hand for chemical potential in both positive and negative exceptional band it follows that

$$
\zeta_{\mathrm{ex}, \pm}^{1}=\left|m_{F}\right|-\lim _{P \rightarrow \infty}\left[\sum_{n=0}^{P} \frac{1}{\sqrt{m_{F}^{2}+2 b(n+1)}}-\int_{0}^{b(P+1)} d w \frac{1}{\sqrt{m_{F}^{2}+2 b n}}\right]
$$

Therefore exceptional band does not split to the first order in $\lambda_{F}$,i.e.,

$$
\Delta_{\text {ex }}=\mathcal{O}\left(\lambda_{F}^{2}\right)
$$

\subsubsection{Perturbation theory in small $\lambda_{B}$}

In this subsection we will move to the opposite end of parameter space from the previous section. We study the gap equations in the limit that $\left|\lambda_{F}\right|$ is near to its largest allowed value, i.e. unity. In this limit the dual t' Hooft coupling, $\lambda_{B}$ is small. In this section we study $\zeta_{\text {ex }}$ at leading order in $\lambda_{B}$ perturbation theory. In this regime of parameters the bosonic theory is weakly coupled and so it is most natural to view the gap equations in bosonic variables. Motivated by this observation, in this section we take $\lambda_{B}$ to zero holding $m_{B}^{\text {cri }}$ fixed. In this limit the gap equations we wish to solve are presented in (4.110), (4.112), (4.114), and (4.116) respectively.

In order to initiate our discussion let us consider, for instance, the equation (4.110). Notice that, in the limit under study in this section, every term in the first two lines of (4.110) has an explicit factor of $\lambda_{B}$. On the other hand the terms in the third line of (4.110) appear with no power of $\lambda_{B}$. In order to find a solution to this equation at small $\lambda_{B}$ we are left with two options. The first option is that $\zeta_{\text {ex }}$ stays finite in the limit $\lambda_{B} \rightarrow 0$. The second option is that $\zeta_{B}$ scales to infinity as $\lambda_{B}$ is taken to zero. Let us consider each of these options in turn.

Option one can only work if the terms in the third line of (4.110) cancel against each other. This can only happen when $M=-1$ and also when $\zeta_{\text {ex }}$ is negative. In this special case we can simply set $\lambda_{B}=0$ in (4.110) and that equation reduces to

$$
-m_{B}^{\mathrm{cri}}=-\left|\zeta_{\mathrm{ex}}\right|+\lim _{P \rightarrow \infty}\left[\sum_{k=0}^{P} \frac{b}{\left(\zeta_{\mathrm{ex}}^{2}+2 b k\right)^{1 / 2}}-\int_{\frac{a^{2}}{2}}^{P b} \frac{d w}{\sqrt{\zeta_{\mathrm{ex}}^{2}+2 w}}\right]
$$

As a slight aside we pause to note that a very similar discussion applies also to the study of the equation (4.112). For that equation as well there exist solutions to the gap equation with $\zeta_{\text {ex }}$ held fixed as $\lambda_{B}$ is taken to zero only when $M=-1$ and when, in this case, $\zeta_{\mathrm{ex}}$ is positive. Indeed the final equation for $\zeta_{\mathrm{ex}}$ in this case reduces exactly to (4.129) (with no modification). Below we will present numerical evidence that suggests that (4.129) does indeed have a (likely unique) solution for $\left|\zeta_{\text {ex }}\right|$ for every value of $m_{B}^{\text {cri }}$.

Note that the condition for existence of 'option one' solutions of (4.110) and (4.112) can be presented in a uniform manner. These solutions exist only for $M=-1$ and also provided

$$
\zeta_{\mathrm{ex}} \lambda_{B}<0
$$


Recall also that the equation (4.110) only applies provided that

$$
\eta \lambda_{B}>0
$$

(here the variable $\eta= \pm 1$ is defined to be positive or negative depending on whether $\mu$ lies in the positive/negative exceptional band) so (4.131) is effectively a third condition for the existence of these solutions.

Let us now return to the consideration of the second option for solutions of (4.110), i.e. the option that we find a solution with $\zeta_{\text {ex }}$ blowing up as $\lambda_{B} \rightarrow 0$. In this limit every term in the second line of (4.110) vanishes faster than $\lambda_{B}$. The l.h.s. of the first line of (4.110) goes to zero like $\lambda_{B}$. On the other hand the r.h.s. of the first line of (4.110) is of order $\lambda_{B}\left|\zeta_{\text {ex }}\right|$. Finally each of the terms in the third line of (4.110) are of order $\left|\zeta_{\text {ex }}\right|$. It follows that the two terms on the third line of (4.110) are dominant in the small $\lambda_{B}$ limit. The only way our gap equation can have a solution is if these two terms cancel against each other upto a residue of order $\lambda_{B}\left|\zeta_{\text {ex }}\right|$. Clearly such a cancellation can only happen if $\zeta_{\text {ex }}$ is negative. Assuming this condition is met, the difference between the two terms on the third line of (4.110) is of order $1 / \sqrt{\left|\zeta_{\mathrm{ex}}\right|}$. This difference can cancel against the term of order $\lambda_{B}\left|\zeta_{\text {ex }}\right|$ only if $\zeta_{\text {ex }}$ is of order $1 / \sqrt{\left|\lambda_{B}\right|}$, more specifically if

$$
\zeta_{\mathrm{ex}}=\frac{\zeta_{\mathrm{ex}}^{-}}{\sqrt{\left|\lambda_{B}\right|}}+\mathcal{O}(1)
$$

The discussion presented of the second option of solutions - i.e. solutions of the gap equation for which $\zeta_{\text {ex }}$ diverges as $\lambda_{B}$ is taken to zero - is easily generalized to the study of the gap equations (4.112), (4.114), and (4.116). In each case we find that solutions of this form occur only when (4.130) is obeyed, and in each case the solutions we seek take the form (4.132).

It is a simple matter to plug (4.132) into the gap equations (4.110), (4.112), (4.114) and (4.116) and to solve for $\zeta_{\mathrm{ex}}^{-}$. Provided $M \neq-1$, in every case we find that

$$
\zeta_{\text {ex }}^{-}=-\operatorname{sgn}\left(\lambda_{\mathrm{B}}\right)\left[\mathrm{b}\left(\mathrm{M}+\frac{3}{2} \mp \frac{\operatorname{sgn}\left(\lambda_{\mathrm{B}}\right)}{2}\right)\right]^{1 / 2}
$$

Where sign $\mp$ is for positive/negative Mth band.

It follows that for $M \neq-1$ the width of the $M^{t h}$ energy state is given by

$$
\Delta_{ \pm}(M)= \pm \frac{b}{\sqrt{\left|\lambda_{B}\right|}}
$$

Note that this (divergent) width is independent of $M$.

In the special case $M=-1$, i.e $\mu$ is in positive/negative exceptional band but does not satisfy the condition in (4.131) (this option only arises in the study of (4.114) and (4.116)), we find

$$
\zeta_{\mathrm{ex}}^{-}=-\operatorname{sgn}\left(\lambda_{\mathrm{B}}\right) \mathrm{b}^{1 / 2}
$$




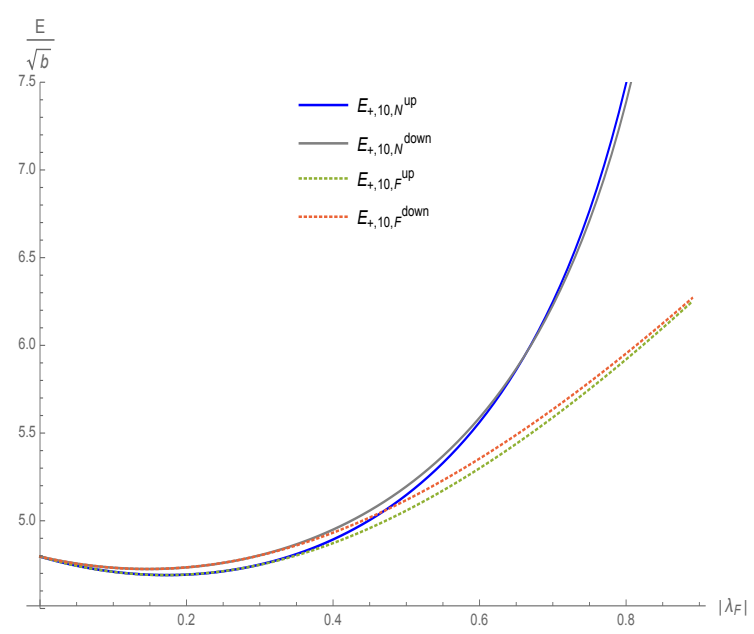

(a) Dashed lines are from fermionic perturbation theory. Solid lines are from numerical analysis.

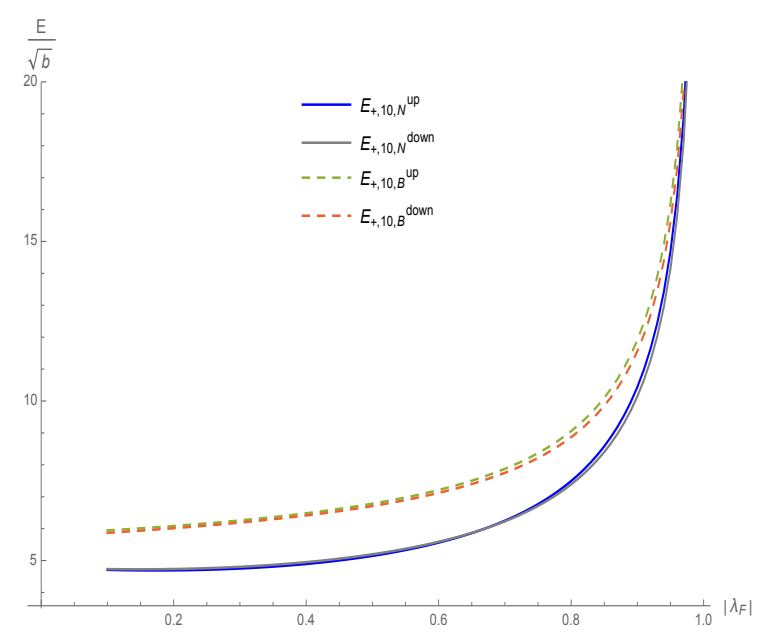

(b) Dashed lines are from bosonic perturbation theory. Solid lines are from numerical analysis.

Figure 2. Upper and lower critical chemical potentials of the $M=10$ positive energy band as function of $\lambda_{F}$ for $\operatorname{sgn}\left(\mathrm{b} \lambda_{\mathrm{F}}\right)=1$ at $m_{B}^{\mathrm{cri}}=-\sqrt{|b|}$.

\subsection{Numerical interpolation between these two regimes}

Although we have been able to solve analytically for $\zeta_{\mathrm{ex}}$ only at small $\lambda_{F}$ or small $\lambda_{B}$, it is not difficult to numerically solve the gap equations for $\zeta_{\mathrm{ex}}$ at any given value of parameters. We have carried out this exercise for a range of different values of parameters.

In this section we briefly present some of our results with the help of a few graphs. The numerical results of this section have all been unsophisticatedly obtained using the numerical equation solving routine on Mathematica. We have not attempted to seriously estimate the errors in our computations, and have presented no error bars on our graphs.

Let us start by noting that the width of the $M^{\text {th }}$ positive level, $\Delta_{+}(M)$ has the same sign as $-\lambda_{F} b$ at small $\lambda_{F}$ (see (4.124)) but has is positive at $\left|\lambda_{F}\right|$ near unity (see (4.134)). It follows that this width must change sign at a finite value $\lambda_{F}$. We illustrate how this happens in figure 2 where we separately plot $E_{+10}^{\text {up }}$ and $E_{+10}^{\text {low }}$ at the fixed value of $m_{B}^{\text {cri }}=-\sqrt{|b|}$, as a function of $\lambda_{F}$. In subfigure 11a we compare our numerical results (subscript $N$ on the graphs) with the results of small $\lambda_{F}$ perturbation theory (subscript $\mathrm{F}$ on the graphs). Note that the agreement is very good at small values of $\lambda_{F}$. Note also that the numerical curves for $E_{+10}^{\mathrm{up}}$ and $E_{+10}^{\text {low }}$ do indeed cross at a value of $\lambda_{F}$ of order 0.7 . In subfigure $11 \mathrm{~b}$ we have plotted the same quantities at larger values of $\lambda_{F}$ and have compared the results with those of small $\lambda_{B}$ perturbation theory (subscript $B$ on the graphs.) Note again the excellent agreement at small $\left|\lambda_{B}\right|$.

In figure 3 we once again plot $E_{+10}^{\text {up }}$ and $E_{+10}^{\text {low }}$ at $m_{B}^{\text {cri }}=-\sqrt{|b|}$, as a function of $\lambda_{F}$, but this time for $\lambda_{F}$ negative. Note that in this case $E_{+10}^{\mathrm{up}}>E_{+10}^{\text {low }}$ for all values of $\lambda_{F}$; the curves never cross. Once again note the excellent agreement between the numerical curves and perturbation theory in its regime of validity.

In figure 4 and figure 5 we turn to the study of negative energy bands. In these two graphs we plot $E_{-10}^{\text {up }}$ and $E_{-10}^{\text {low }}$ (note that $E_{-10}^{\text {up }}<E_{-10}^{\text {low }}$ but $\left|E_{-10}^{\text {up }}\right|>\left|E_{-10}^{\text {low }}\right|$ ) as a function of 


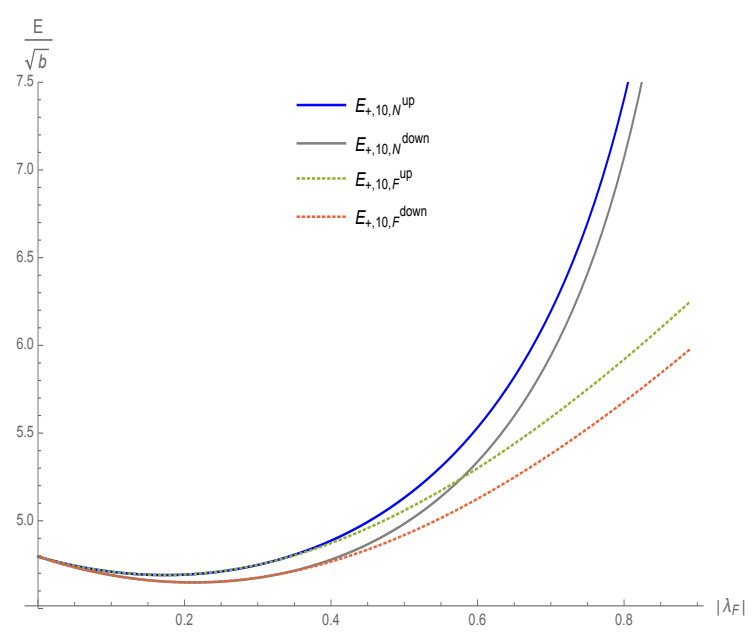

(a) Dashed lines are from fermionic perturbation theory. Solid lines are from numerical analysis.

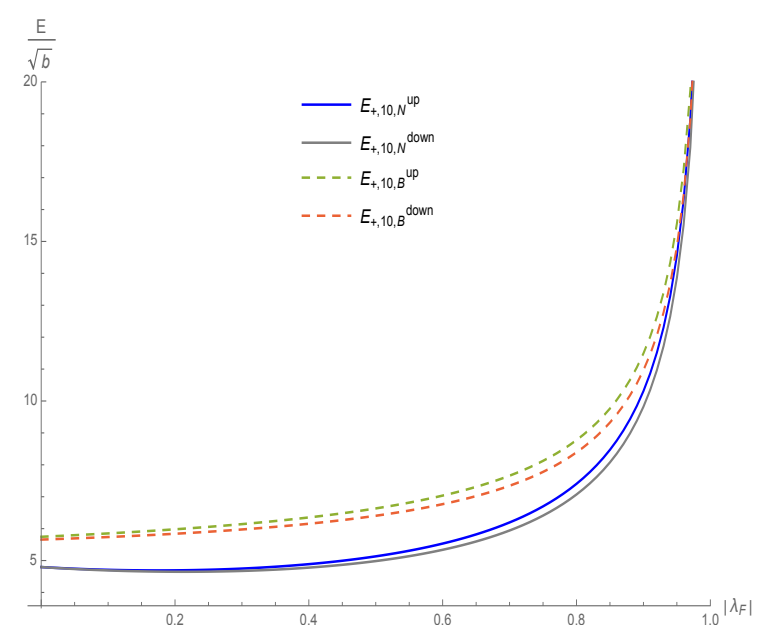

(b) Dashed lines are from bosonic perturbation theory. Solid lines are from numerical analysis.

Figure 3. Upper and lower critical chemical potentials for the $M=10$ positive energy band function of $\lambda_{F}$ for $\operatorname{sgn}\left(\mathrm{b} \lambda_{\mathrm{F}}\right)=-1$, at fixed $m_{B}^{\text {cri }}=-\sqrt{|b|}$.

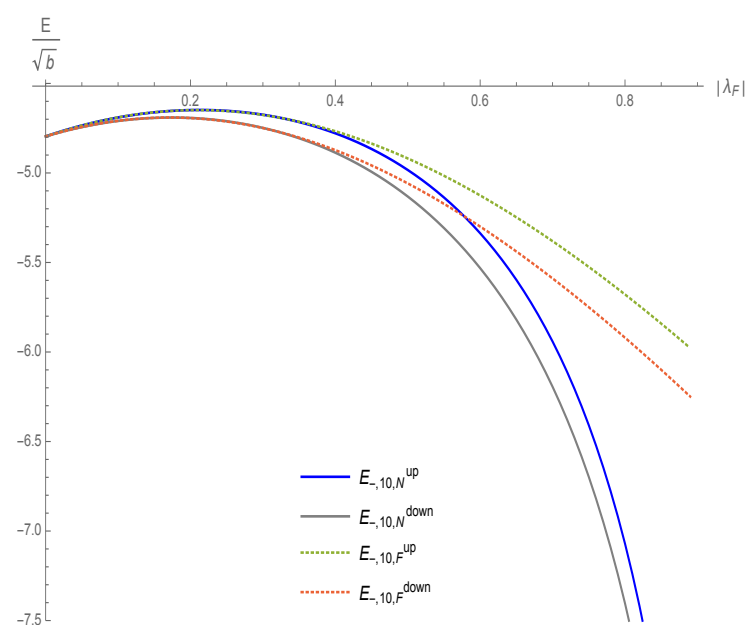

(a) Dashed lines are from fermionic perturbation theory. Solid lines are from numerical analysis.

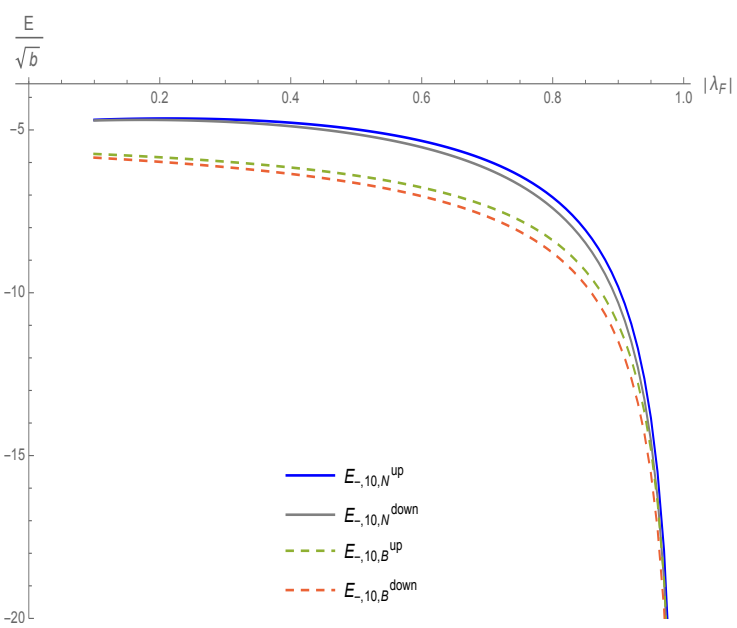

(b) Dashed lines are from bosonic perturbation theory. Solid lines are from numerical analysis.

Figure 4. Critical chemical potentials for the $M=10$ negative energy band as a function of $\lambda_{F}$ for $\operatorname{sgn}\left(\mathrm{b} \lambda_{\mathrm{F}}\right)=1$ at $m_{B}^{\text {cri }}=-\sqrt{|b|}$.

$\lambda_{F}$ respectively for positive and negative values of $\lambda_{F} b$. As could have been anticipated by comparing (4.126) and (4.134), in this case the curves cross when $\lambda_{F} b$ is negative (figure 5) but not when it is positive (figure 4). Note again the excellent agreement with perturbation theory in the appropriate parameter ranges.

Finally we turn to a study of the exceptional bands. In figures $6,7,8$ and 9 respectively we plot the critical energies $E_{\mathrm{ex}}^{\mathrm{up}}$ a and $E_{\mathrm{ex}}^{\text {down }}$ of the exceptional energy band the following four cases. First when $\operatorname{sgn}\left(\lambda_{F} b\right)=1$ and $\operatorname{sgn}\left(M_{F} \lambda_{F}\right)=1$. Second when $\operatorname{sgn}\left(\lambda_{F} b\right)=$ -1 and $\operatorname{sgn}\left(M_{F} \lambda_{F}\right)=1$. Third when $\operatorname{sgn}\left(\lambda_{F} b\right)=1$ and $\operatorname{sgn}\left(M_{F} \lambda_{F}\right)=-1$. Fourth 


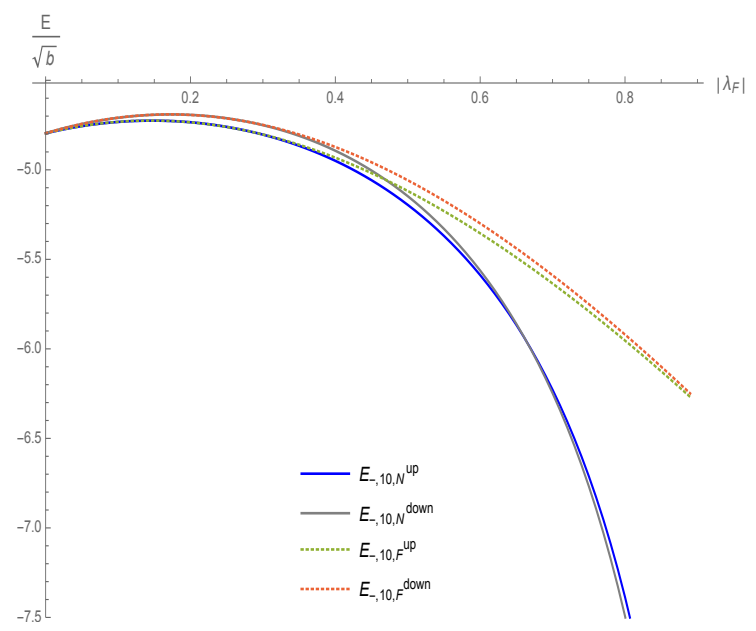

(a) Dashed lines are from fermionic perturbation theory. Solid lines are from numerical analysis.

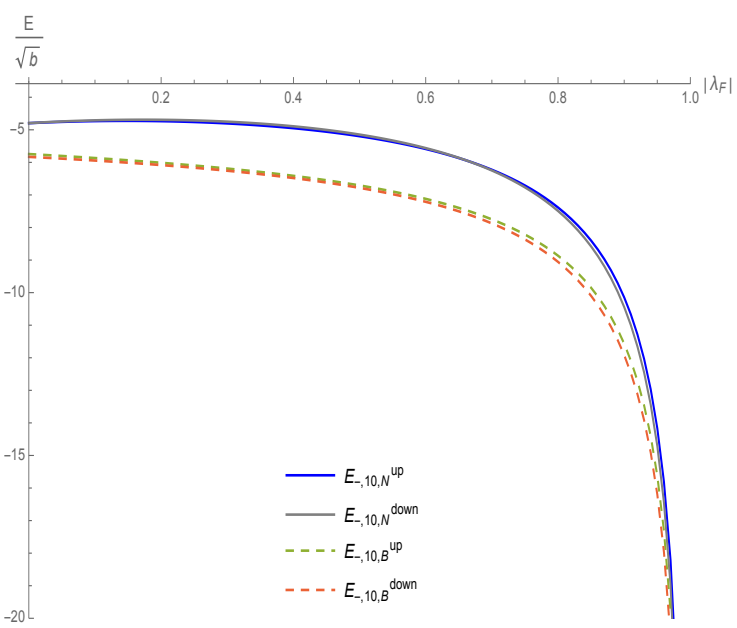

(b) Dashed lines are from bosonic perturbation theory. Solid lines are from numerical analysis.

Figure 5. The critical chemical potentials for the $M=10$ negative energy band as a function of $\lambda_{F}$ for $\operatorname{sgn}\left(\mathrm{b} \lambda_{\mathrm{F}}\right)=-1$ at fixed $m_{B}^{\text {cri }}=-\sqrt{|b|}$.

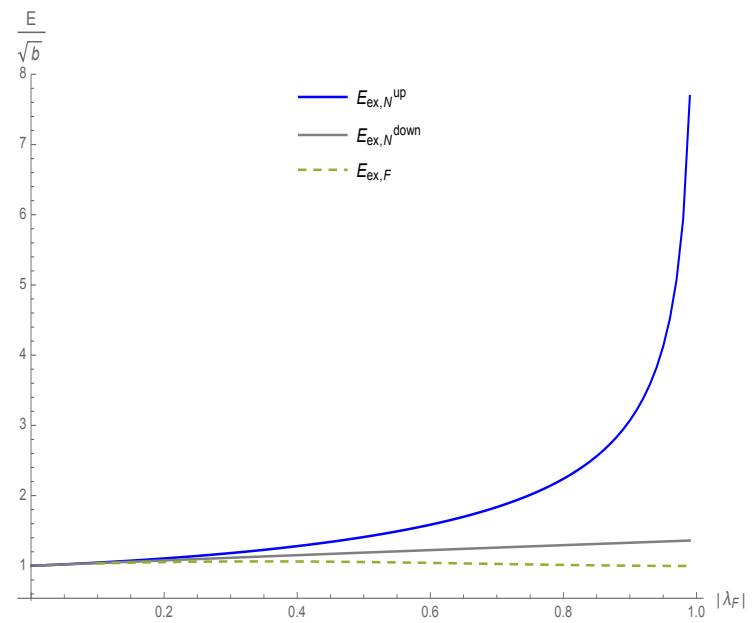

(a) Dashed lines are from fermionic perturbation theory. Solid lines are from numerical analysis.

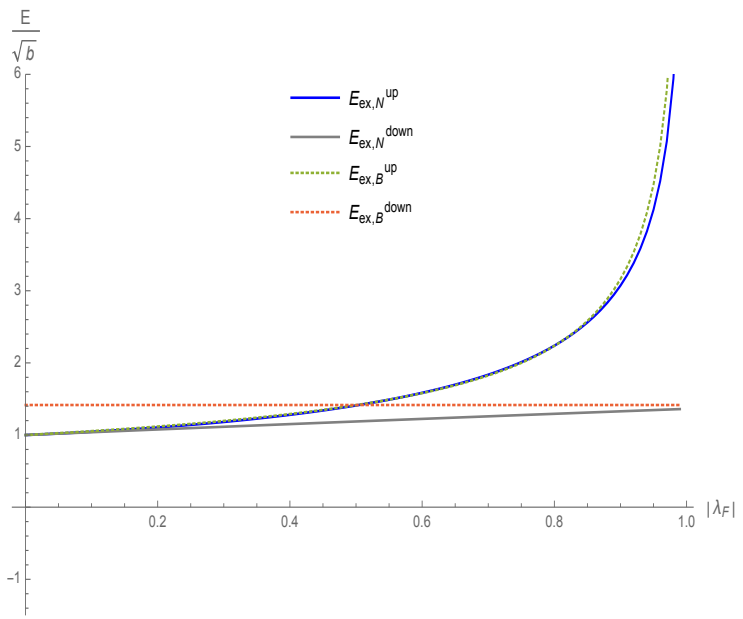

(b) Dashed lines are from bosonic perturbation theory. Solid lines are from numerical analysis.

Figure 6. Exceptional energy band as a function of $\lambda_{F}$ for $\operatorname{sgn}\left(\mathrm{b} \lambda_{\mathrm{F}}\right)=1, \operatorname{sgn}\left(\mathrm{m}_{\mathrm{F}} \lambda_{\mathrm{F}}\right)=1$ at $m_{B}^{\text {cri }}=\sqrt{|b|}$.

when $\operatorname{sgn}\left(\lambda_{F} b\right)=-1$ and $\operatorname{sgn}\left(M_{F} \lambda_{F}\right)=-1$. Note again the reasonable agreement with perturbation theory. Note also that in every case that $E_{\mathrm{ex}}^{\text {down }}$ is positive at small $\left|\lambda_{B}\right|$, it remains finite as $\left|\lambda_{B}\right| \rightarrow 0$, while in every case that $E_{\mathrm{ex}}^{\mathrm{up}}$ is negative at small $\lambda_{B}$, it remains finite as $\left|\lambda_{B}\right| \rightarrow 0$. Note also that the curves cut the $\mathrm{x}$ axis in the last two cases. 


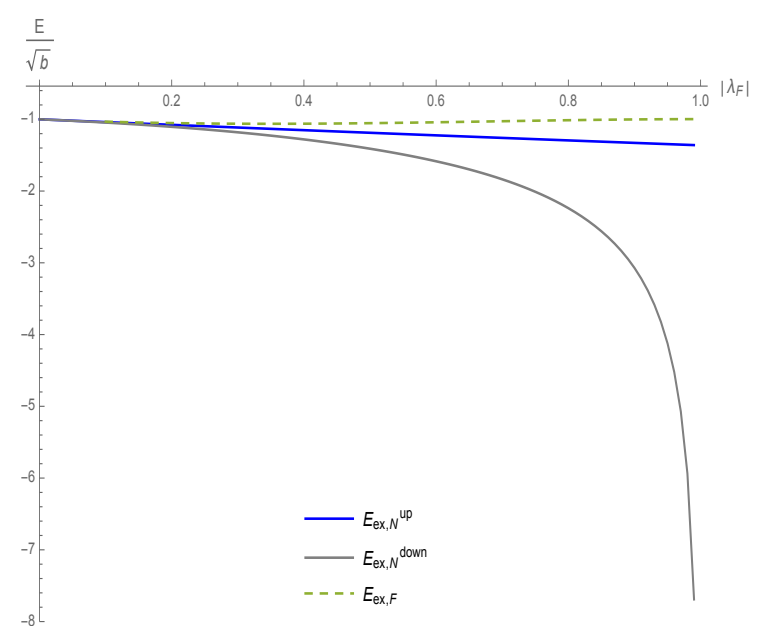

(a) Dashed lines are from fermionic perturbation theory. Solid lines are from numerical analysis.

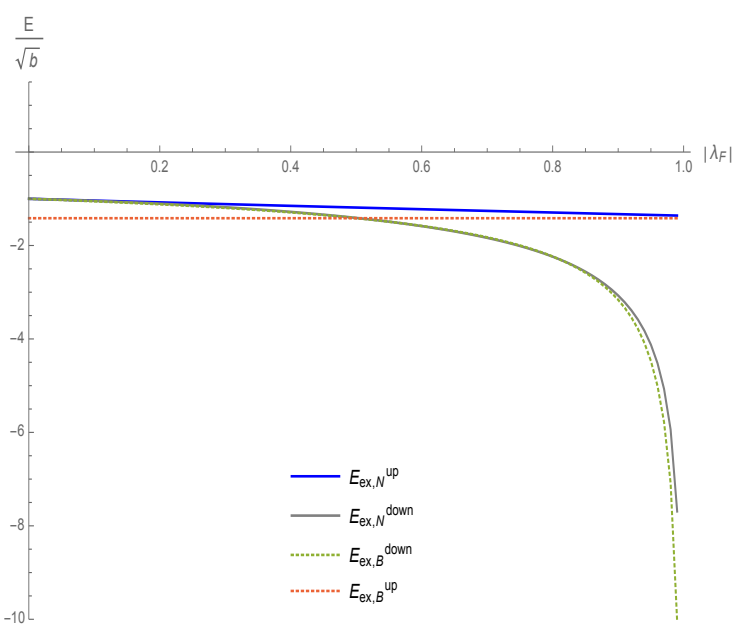

(b) Dashed lines are from bosonic perturbation theory. Solid lines are from numerical analysis.

Figure 7. Exceptional energy band as a function of $\lambda_{F}$ for $\operatorname{sgn}\left(b \lambda_{F}\right)=-1, \operatorname{sgn}\left(m_{F} \lambda_{F}\right)=1$ at $m_{B}^{\text {cri }}=\sqrt{|b|}$.

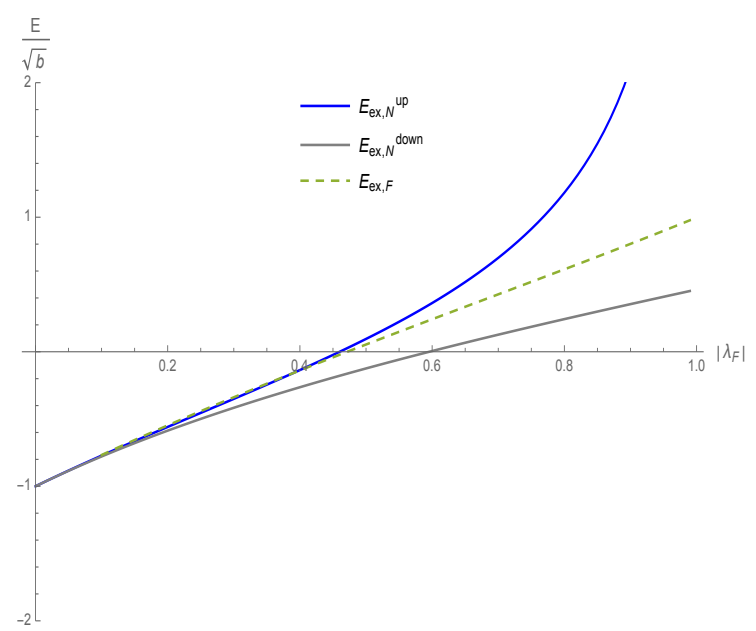

(a) Dashed lines are from fermionic perturbation theory. Solid lines are from numerical analysis.

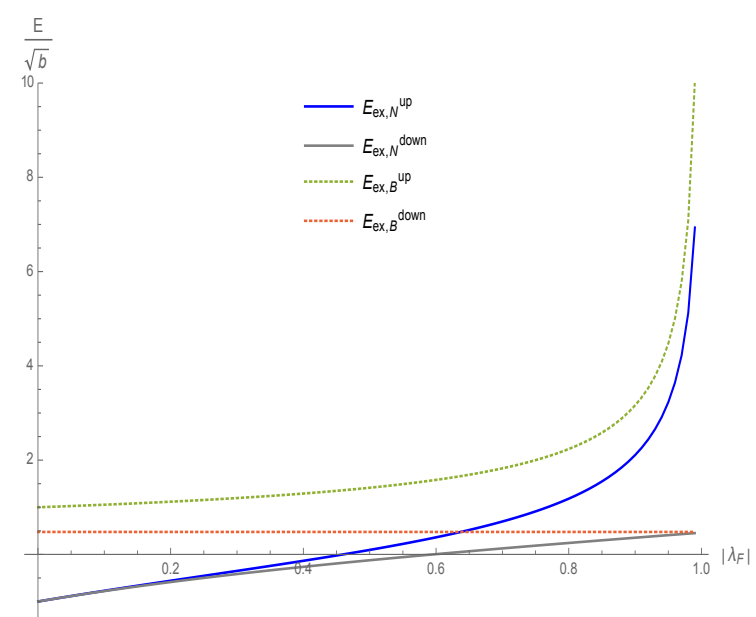

(b) Dashed lines are from bosonic perturbation theory. Solid lines are from numerical analysis.

Figure 8. Exceptional energy band as a function of $\lambda_{F}$ for $\operatorname{sgn}\left(\mathrm{b} \lambda_{\mathrm{F}}\right)=1, \operatorname{sgn}\left(\mathrm{m}_{\mathrm{F}} \lambda_{\mathrm{F}}\right)=-1$ at $m_{B}^{\text {cri }}=-\sqrt{|b|}$.

\section{Regular bosons coupled with Chern Simons gauge field}

In the previous section we have presented an exhaustive analysis of Greens function and the single particle eigen energies for the regular fermion theory in a magnetic field background, both with and without a chemical potential. As we have reviewed in the introduction, however, the regular fermion theory is conjectured to be dual to the theory of critical bosons. As a check both of this conjectured duality — but also of the fermionic results of the previous section — in this section we study the propagator and single particle energy 


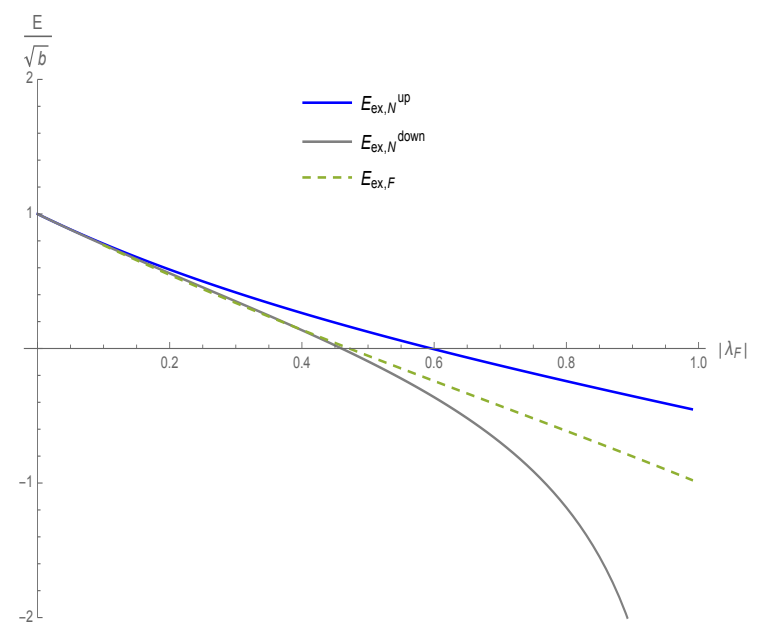

(a) Dashed lines are from fermionic perturbation theory. Solid lines are from numerical analysis.

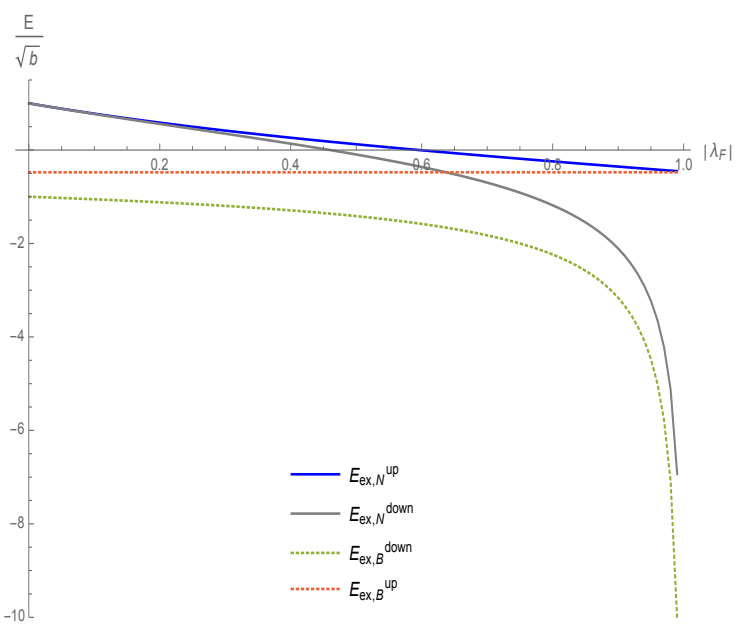

(b) Dashed lines are from bosonic perturbation theory. Solid lines are from numerical analysis.

Figure 9. Exceptional energy band as a function of $\lambda_{F}$ for $\operatorname{sgn}\left(b \lambda_{\mathrm{F}}\right)=-1, \operatorname{sgn}\left(\mathrm{m}_{\mathrm{F}} \lambda_{\mathrm{F}}\right)=-1$ at $m_{B}^{\mathrm{cri}}=-\sqrt{|b|}$.

eigenstates of the critical boson theory in the background of a global symmetry magnetic field. For the reason explained in the introduction, in this section (i.e. the relative difficult of solving the gap equations in the presence of a Bose condensate) in this section we work only at zero chemical potential, leaving the generalization to nonzero chemical potential to future work.

\subsection{Gap equations}

We consider following Euclidean action for a fundamental boson coupled with Chern Simons gauge field

$$
S_{B}=\frac{i \kappa_{B}}{4 \pi} \int \operatorname{Tr}\left(A d A-\frac{2 i}{3} A^{3}\right)+\int\left(\tilde{D}^{\mu} \bar{\phi} \tilde{D}_{\mu} \phi+m_{B}^{2} \bar{\phi} \phi+\frac{4 \pi b_{4}}{k}(\bar{\phi} \phi)^{2}+\frac{4 \pi^{2} x_{6}}{k^{2}}(\bar{\phi} \phi)^{3}\right)
$$

Here $A$ is a $\mathrm{U}\left(N_{B}\right)$ connection, and we have turned on a suitably normalized background (topological) $\mathrm{U}(1)$ gauge field $a_{\mu}$. In the large $N_{B}$ limit under study the fluctuations of the $\mathrm{U}(1)$ part of the gauge field are subdominant in a $\frac{1}{N_{B}}$ expansion, and so the $\mathrm{U}(1)$ part of the $\mathrm{U}\left(N_{B}\right)$ gauge field can effectively be replaced by its saddle point value. This saddle point value is obtained from the equation of motion of the $\mathrm{U}(1)$ gauge field in the presence of the source coupling to the topological current. Ignoring the matter contribution to this equation of motion (which we know to be suppressed in the large $N_{B}$ limit) and with a suitable choice of normalization for $a$ we find that the equation of motion simply identifies the U(1) part of the dynamical gauge field with $a_{\mu}$. When the dust settles, to the accuracy we work in this paper, the bosons can be thought of as charged under the gauge group $\mathrm{SU}\left(N_{B}\right)$ and the covariant derivative that appears in (5.1) may be thought of as acting as

$$
\tilde{D}_{\mu} \phi=\left(\partial_{\mu}-i A_{\mu}-i a_{\mu}\right) \phi
$$

in close analogy with the previous section. 


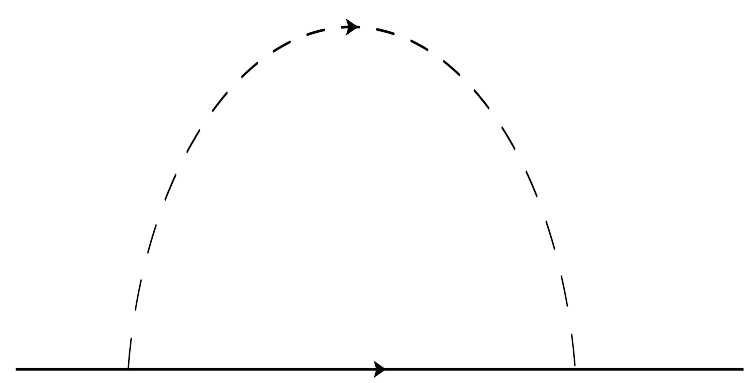

(a) Contribution of this diagram is presented in (5.7). Solid lines are scalar propagators while dashed lines are gauge boson propagators.

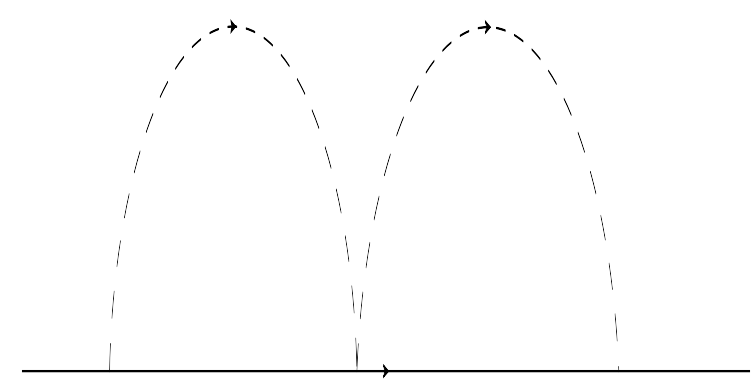

(b) Contribution of this diagram is presented in (5.8). Solid lines scalar field propagators while dotted lines are gauge boson propagators.

Figure 10. The 'one loop' and one of the 'two loop' graphs that contribute to the bosonic self energy. Note that the contribution of the one loop graph vanishes in the absence of a magnetic field.

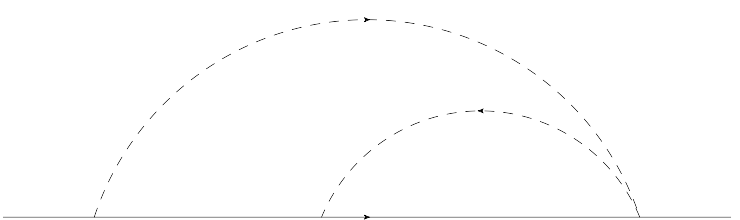

(a) Contribution of this diagram is presented in (5.9). Solid lines are scalar propagators while dashed lines are gauge boson propagators.

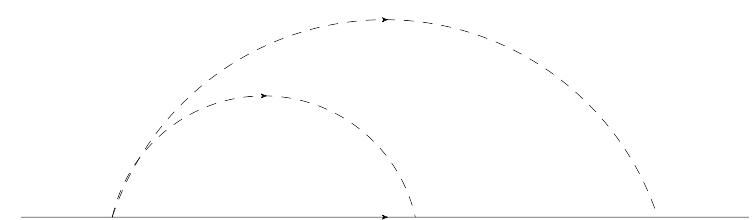

(b) Contribution of this diagram is presented in (5.10). Solid lines are scalar propagators while dotted lines are gauge boson propagators.

Figure 11. The remaining two 'two loop' graphs that contribute to the bosonic self energy. The two graphs above contribute identically in the $b=0$ limit, but are distinct at nonzero $b$. While each of the graphs drawn above is individually complicated, their sum turns out to be relatively simple.

Below we study the theory leading order in large $N$ approximation, but all order in $\mathrm{t}$ 'Hooft coupling $\lambda_{B}=\frac{N_{B}}{\kappa_{B}}$. The saddle point equations in $A_{-}=0$ gauge (See appendix $\mathrm{H}$ for details) take the form

$$
\left(-D_{x}^{2}+m_{B}^{2}\right) \alpha(x, y)+\int d^{3} z \Sigma(x, z) \alpha(z, y)=\delta^{(3)}(x-y)
$$

where $D_{\mu}$ is the 'background covariant derivative'

$$
D_{\mu} \phi=\left(\partial_{\mu}-i a_{\mu}\right) \phi
$$

As in the fermionic theory, the self energy $\Sigma(x, y)$ is the sum of a set of one (and in this case also two) loop diagrams graphs built using the exact propagator $\alpha$. We find

$$
\Sigma(x, y)=\Sigma_{1}\left(x_{T}, x_{R}\right)+\Sigma_{2 A}\left(x_{T}, x_{R}\right)+\Sigma_{2 B}\left(x_{T}, x_{R}\right)+\Sigma_{2 B^{\prime}}\left(x_{T}, x_{R}\right)
$$

where the quantities $\Sigma_{1}, \Sigma_{2 A} \Sigma_{2 B}$ and $\Sigma_{2 C}$ are the expressions computed by the Feynman graphs presented in figures 10,11 .

The coordinates $x_{T}$ and $x_{R}$ that appear in (5.5) refer respectively to centre of mass and relative coordinates

$$
x_{T}=x+y, \quad x_{R}=x-y
$$


The explicit expressions for $\Sigma_{1}, \Sigma_{2 A} \Sigma_{2 B}$ and $\Sigma_{2 C}$ take the form

$$
\begin{aligned}
\Sigma_{1}\left(x_{T}, x_{R}\right)=8 \pi \lambda_{B}\left(b_{4} \alpha\left(x_{T}, x_{R}\right) \delta^{(3)}\left(x_{R}\right)+i \partial_{x_{T}^{-}}\right. & \partial_{x_{R}^{3}}\left(G\left(x_{T}, x_{R}\right) \alpha\left(x_{T}, x_{R}\right)\right) \\
& \left.\left.+G\left(x_{T}, x_{R}\right) \partial_{x_{R}^{3}} \alpha\left(x_{T}, x_{R}\right)\right)\right)
\end{aligned}
$$

$$
\begin{aligned}
& \Sigma_{2 A}\left(x_{T}, x_{R}\right)=4 \pi^{2} \lambda_{B}^{2}\left(x_{6} \alpha\left(x_{T}, x_{R}\right)^{2} \delta^{(3)}\left(x_{R}\right)+\alpha\left(x_{T}, x_{R}\right)^{2} \delta^{(3)}\left(x_{R}\right)\right. \\
& \quad+4 i \partial_{x_{R}^{-}} \int d^{3} z \alpha\left(x_{1, T}, x_{1, R}\right) \delta^{(3)}\left(x_{1, R}\right) G\left(x_{2, T}, x_{2, R}\right) \alpha\left(x_{2, T}, x_{2, R}\right) \\
& \left.\quad-4\left(\partial_{x_{R}^{-}}+\partial_{x_{T}^{-}}\right)\left(\partial_{x_{R}^{-}}-\partial_{x_{T}^{-}}\right) \int d^{3} z \alpha\left(x_{1, T}, x_{1, R}\right) G\left(x_{1, T}, x_{1, R}\right) \alpha\left(x_{2, T}, x_{2, R}\right) G\left(x_{2, T}, x_{2, R}\right)\right)
\end{aligned}
$$

$$
\begin{aligned}
& \Sigma_{2 B}\left(x_{T}, x_{R}\right)=4 \pi^{2} \lambda_{B}^{2}\left(x_{6} \alpha\left(x_{T}, x_{R}\right)^{2} \delta^{(3)}\left(x_{R}\right)\right. \\
& \quad+4\left(\partial_{x_{R}^{-}}+\partial_{x_{T}^{-}}\right)\left(\partial_{x_{R}^{-}}+\partial_{x_{T}^{-}}\right) \int d^{3} z \alpha\left(x_{1, T}, x_{1, R}\right) G\left(x_{T}, x_{R}\right) \alpha\left(x_{2, T}, x_{2, R}\right) G\left(x_{2, T}, x_{2, R}\right) \\
& \quad-6\left(\partial_{x_{R}^{-}}+\partial_{x_{T}^{-}}\right) \int d^{3} z \alpha\left(x_{1, T}, x_{1, R}\right) \partial_{x_{R}^{-}} G\left(x_{T}, x_{R}\right) \alpha\left(x_{2, T}, x_{2, R}\right) G\left(x_{2, T}, x_{2, R}\right) \\
& \quad-2\left(\partial_{x_{R}^{-}}+\partial_{x_{T}^{-}}\right) \int d^{3} z \alpha\left(x_{1, T}, x_{1, R}\right) G\left(x_{T}, x_{R}\right) \alpha\left(x_{2, T}, x_{2, R}\right) \partial_{x_{2, R}^{-}} G\left(x_{2, T}, x_{2, R}\right) \\
& \quad-8\left(\partial_{x_{R}^{-}}+\partial_{x_{T}^{-}}\right) \int d^{3} z \partial_{x_{1, T}^{-}} \alpha\left(x_{1, T}, x_{1, R}\right) G\left(x_{T}, x_{R}\right) \alpha\left(x_{2, T}, x_{2, R}\right) G\left(x_{2, T}, x_{2, R}\right) \\
& \quad+4 \int d^{3} z \partial_{x_{1, T}^{-}} \alpha\left(x_{1, T}, x_{1, R}\right) \partial_{x_{R}^{-}} G\left(x_{T}, x_{R}\right) \alpha\left(x_{2, T}, x_{2, R}\right) G\left(x_{2, T}, x_{2, R}\right) \\
& \quad+2 \int d^{3} z \alpha\left(x_{1, T}, x_{1, R}\right) \partial_{x_{R}^{-}}^{2} G\left(x_{T}, x_{R}\right) \alpha\left(x_{2, T}, x_{2, R}\right) G\left(x_{2, T}, x_{2, R}\right) \\
& \left.\quad+\int d^{3} z \alpha\left(x_{1, T}, x_{1, R}\right) \partial_{x_{R}^{-}} G\left(x_{T}, x_{R}\right) \alpha\left(x_{2, T}, x_{2, R}\right) \partial_{x_{2, R}^{-}} G\left(x_{2, T}, x_{2, R}\right)\right)
\end{aligned}
$$

$$
\begin{aligned}
& \Sigma_{2 B^{\prime}}\left(x_{T}, x_{R}\right)=4 \pi^{2} \lambda_{B}^{2}\left(x_{6} \alpha\left(x_{T}, x_{R}\right)^{2} \delta^{(3)}\left(x_{R}\right)\right. \\
& \quad+4\left(\partial_{x_{R}^{-}}-\partial_{x_{T}^{-}}\right)\left(\partial_{x_{R}^{-}}+\partial_{x_{T}^{-}}\right) \int d^{3} z \alpha\left(x_{1, T}, x_{1, R}\right) G\left(x_{1, T}, x_{1, R}\right) \alpha\left(x_{2, T}, x_{2, R}\right) G\left(x_{T}, x_{R}\right) \\
& \quad-2\left(\partial_{x_{R}^{-}}+\partial_{x_{T}^{-}}\right) \int d^{3} z \alpha\left(x_{1, T}, x_{1, R}\right) G\left(x_{1, T}, x_{1, R}\right) \alpha\left(x_{2, T}, x_{2, R}\right) \partial_{x_{R}^{-}} G\left(x_{T}, x_{R}\right) \\
& \quad-4\left(\partial_{x_{R}^{-}}-\partial_{x_{T}^{-}}\right) \int d^{3} z \alpha\left(x_{1, T}, x_{1, R}\right) G\left(x_{1, T}, x_{1, R}\right) \alpha\left(x_{2, T}, x_{2, R}\right) \partial_{x_{R}^{-}} G\left(x_{T}, x_{R}\right) \\
& \quad-2\left(\partial_{x_{R}^{-}}-\partial_{x_{T}^{-}}\right) \int d^{3} z \alpha\left(x_{1, T}, x_{1, R}\right) \partial_{x_{1, R}^{-}} G\left(x_{1, T}, x_{1, R}\right) \alpha\left(x_{2, T}, x_{2, R}\right) G\left(x_{T}, x_{R}\right) \\
& \quad-8\left(\partial_{x_{R}^{-}}-\partial_{x_{T}^{-}}\right) \int d^{3} z \partial_{x_{1, T}^{-}} \alpha\left(x_{1, T}, x_{1, R}\right) G\left(x_{1, T}, x_{1, R}\right) \alpha\left(x_{2, T}, x_{2, R}\right) G\left(x_{T}, x_{R}\right) \\
& \quad+4 \int d^{3} z \partial_{x_{1, T}^{-}} \alpha\left(x_{1, T}, x_{1, R}\right) \partial_{x_{R}^{-}} G\left(x_{T}, x_{R}\right) \alpha\left(x_{2, T}, x_{2, R}\right) G\left(x_{1, T}, x_{1, R}\right) \\
& \quad+2 \int d^{3} z \alpha\left(x_{1, T}, x_{1, R}\right) \partial_{x_{R}^{-}}^{2} G\left(x_{T}, x_{R}\right) \alpha\left(x_{2, T}, x_{2, R}\right) G\left(x_{1, T}, x_{1, R}\right) \\
& \left.\quad+\int d^{3} z \alpha\left(x_{1, T}, x_{1, R}\right) \partial_{x_{R}^{-}} G\left(x_{T}, x_{R}\right) \alpha\left(x_{2, T}, x_{2, R}\right) \partial_{x_{2, R}^{-}} G\left(x_{1, T}, x_{1, R}\right)\right)
\end{aligned}
$$


In the integral expressions above $z$ represents the position of the interaction vertex which is integrated over all of spacetime. In each of the expressions above we have used the following notation (see appendix A)

$$
x_{1, T}=x+z, \quad x_{1, R}=x-z, \quad x_{2, T}=z+y, \quad x_{2, R}=z-y
$$

\subsection{Constant magnetic field}

As in the previous section, we wish to study our theory in the presence of a background gauge field of the special form

$$
a_{j}=-b \frac{\epsilon_{j i} x^{i}}{2}
$$

It follows from the discussion of section 2.1.1 that

$$
\alpha(x, y)=e^{-i \frac{b}{2}\left(x^{1} y^{2}-x^{2} y^{1}\right)} \alpha_{R}(x-y), \quad \Sigma(x, y)=e^{-i \frac{b}{2}\left(x^{1} y^{2}-x^{2} y^{1}\right)} \Sigma_{R}(x-y)
$$

where the functions $\alpha_{R}(x-y)$ and $\Sigma_{R}(x-y)$ are both simultaneously rotationally and translationally invariant. One difference with the previous section is that, in this case, $\Sigma_{R}(x-y)$ and $\alpha_{R}(x-y)$ are both index free scalar functions. It follows that $\Sigma_{R}(x-y)$ and $\alpha_{R}(x-y)$ admit a much simpler expansion in terms of the functions $e_{m, n}$ than in the previous section. Explicitly we have

$$
\begin{aligned}
\alpha_{R}(x) & =\int_{-\infty}^{\infty} \frac{d w}{2 \pi} e^{i \omega t} \alpha_{R}(\omega, \vec{x}), & \alpha_{R}(\omega, \vec{x}) & =\sum_{n=0}^{\infty} \alpha_{n}(\omega) e_{n, n}(\vec{x}) \\
\Sigma_{R}(x) & =\int_{-\infty}^{\infty} \frac{d w}{2 \pi} e^{i \omega t} \Sigma(\omega, \vec{x}), & \Sigma_{R}(\omega, \vec{x}) & =\sum_{n=0}^{\infty} \Sigma_{n}(\omega) e_{n, n}(\vec{x}) .
\end{aligned}
$$

Note that the r.h.s. of has terms of at most first order in $x_{R}^{3}$. It follows from this observation that the contribution of $\Sigma_{1}$ to the quantity $\left.\Sigma_{(} \omega, \vec{x}\right)$ defned in (5.14) is a polynomial of first degree in $\omega$. On the other hand the expressions on the r.h.s. of (5.8), (5.9) and (5.10) each have no derivatives in $x_{R}^{3}$; it follows that the contributions of the $\Sigma_{2 A}, \Sigma_{2 B}$ and $\Sigma_{2 B^{\prime}}$ to $\left.\Sigma_{(} \omega, \vec{x}\right)$ are all independent of $\omega$. In summary $\left.\Sigma_{(} \omega, \vec{x}\right)$ is a polynomial of unit degree in $\omega$. It follows that the second expansion on the second line of (5.14) is completely specified by two expansion coefficients (the coefficient of the constant and the coefficient of the term proportional to $\omega$ ) at every value of $n$. We find it convenient to parameterize these two coefficients in terms of the coefficients $\zeta_{+}^{B}(n)$ and $\zeta_{-}^{B}(n)$ defined by

$$
\Sigma_{n}(\omega)=-i \omega\left(\zeta_{+}^{B}(n)-\zeta_{-}^{B}(n)\right)+\zeta_{+}^{B}(n) \zeta_{-}^{B}(n)-b(2 n+1)-m_{B}^{2}
$$

The form of the parameterization (5.15) ensures that the gap equation (5.3) has the following simple 'solution' in terms of $\zeta_{+}^{B}(n)$ and $\zeta_{-}^{B}(n)$

$$
\alpha_{R}(\omega, \vec{x})=\sum_{n=0}^{\infty} \frac{1}{\left(\omega-i \zeta_{+}^{B}(n)\right)\left(\omega+i \zeta_{-}^{B}(n)\right)} e_{n, n}(\vec{x})
$$


The second gap equation (5.5) - after a considerable amount of algebraic manipulation can be massaged into the form (see appendix for details)

$$
\begin{aligned}
\Sigma_{R}(\omega, \vec{x}) & =\Sigma_{R, 0}(\omega, \vec{x})+\Sigma_{R, 1}(\omega, \vec{x})+\Sigma_{R, 2}(\omega, \vec{x}) \\
\Sigma_{R, 0}(\omega, \vec{x}) & =8 \pi \lambda_{B} b_{4} \delta^{(2)}(\vec{x}) \int \frac{d \omega^{\prime}}{2 \pi} \alpha_{R}\left(\omega^{\prime}, \overrightarrow{0}\right)+4 \pi^{2} \lambda_{B}^{2}\left(1+3 x_{6}\right)\left(\int \frac{d \omega^{\prime}}{2 \pi} \alpha_{R}\left(\omega^{\prime}, \overrightarrow{0}\right)\right)^{2} \delta^{(2)}(\vec{x}) \\
\Sigma_{R, 1}(\omega, \vec{x}) & =-2 \lambda_{B} b\left(i \omega \int \frac{d \omega^{\prime}}{2 \pi} \alpha_{R}\left(\omega^{\prime}, \vec{x}\right)+\int \frac{d \omega^{\prime}}{2 \pi} i \omega^{\prime} \alpha_{R}\left(\omega^{\prime}, \vec{x}\right)\right) \\
\Sigma_{R, 2}(\omega, \vec{x}) & =-\left(2 \lambda_{B} b\right)^{2}\left(\int \frac{d \omega_{1}^{\prime}}{2 \pi} \frac{d \omega_{2}^{\prime}}{2 \pi} \alpha_{R}\left(\omega_{1}^{\prime}, \vec{x}\right) *_{b} \alpha_{R}\left(\omega_{2}^{\prime}, \vec{x}\right)\right)
\end{aligned}
$$

where $*_{b}$ is the twisted convolution defined in (3.1).

In the rest of this section we will recast the gap equations (5.16) and (5.17) into equations for $\zeta_{ \pm}^{B}(n)$ and then proceed to solve these equations.

\subsection{Solving the gap equations}

In this subsection we study the gap equations obtained in previous section. We proceed by plugging $\alpha$ from (5.16) to r.h.s. of (5.17) and performing $\omega$ integrals. To simplify the star product appearing in (5.17) we use property presented in (3.2) of the basis elements $e_{m, n}$.

As we have explained above, of the three terms on the r.h.s. of the first line of (5.17), only the term $\Sigma_{R, 1}$ has a contribution proportional to $\omega$. This term - i.e. the term proportional to $\omega-$ arises from the first integral on the r.h.s. of third line in (5.17). The integral in question is easily verified to be proportional to $\frac{1}{\zeta_{+}^{B}(n)+\zeta_{-}^{B}(n)}$. Equating this integral to the coefficient of $\omega$ in (5.15) we obtain the equation

$$
\zeta_{+}^{B}(n)^{2}-\zeta_{-}^{B}(n)^{2}=2 \lambda_{B} b
$$

Evaluating the remaining integrals in (5.17) gives us an expression for the $\omega$ independent part of $\Sigma_{n}(\omega)$. Collecting terms and comparing equating with the $\omega$ independent part of (5.15) we find the second equation

$$
\zeta_{+}^{B}(n)^{2}+\zeta_{-}^{B}(n)^{2}=2\left(c_{B}^{2}+b(2 n+1)\right)
$$

where we have defined the quantity

$$
c_{B}^{2}=m_{B}^{2}+8 \pi \lambda_{B} b_{4} \int \frac{d \omega^{\prime}}{2 \pi} \alpha_{R}\left(\omega^{\prime}, \overrightarrow{0}\right)+4 \pi^{2} \lambda_{B}^{2}\left(1+3 x_{6}\right)\left(\int \frac{d \omega^{\prime}}{2 \pi} \alpha_{R}\left(\omega^{\prime}, \overrightarrow{0}\right)\right)^{2}
$$

(5.18), (5.19) are readily solved to give

$$
\zeta_{ \pm}^{B}(n)^{2}=c_{B}^{2}+b(2 n+1) \pm \lambda_{B} b
$$

The quantity $c_{B}$ is now determined by plugging (5.16) and (5.21) into (5.20) and solving the resultant equation for $c_{B}$. 


\subsection{Critical scaling limit and the duality}

In order to match with the fermionic results of the previous section we are interested in the theory (5.1) in the so called critical boson scaling limit defined by scaling $m_{B}^{2}$ and $b_{4}$ to $\infty$ while keeping the following quantity fixed

$$
m_{B}^{\text {cri }}=\frac{m_{B}^{2}}{2 \lambda_{B} b_{4}}
$$

In this limit equation (5.20) simplifies to

$$
4 \pi \lambda_{B} \int \frac{d \omega^{\prime}}{2 \pi} \alpha_{R}\left(\omega^{\prime}, \overrightarrow{0}\right)=-m_{B}^{\mathrm{cri}} \lambda_{B}
$$

When this condition is maintained $c_{B}$ defined above has a finite value in the scaling limit under consideration. Using (5.16) and (5.18) this equation can be simplified to

$$
\sum_{n=0}^{\infty}\left(\zeta_{+}^{B}(n)-\zeta_{-}^{B}(n)\right)=-m_{B}^{\mathrm{cri}} \lambda_{B}
$$

The equation (5.24) is formal as the summation on l.h.s. of this equation is divergent. As in the previous section, this divergence should be regulated by adding the same counterterm to the theory that one adds to renormalize the corresponding divergence in the absence of a magnetic field. As in the previous subsection this is achieved by adding and subtracting the relevant integral. Our gap equation turns into

$$
\begin{aligned}
& -\lambda_{B} m_{B}^{\mathrm{cri}}=\lambda_{B} \int_{a}^{\infty} \frac{p_{s} d p_{s}}{\sqrt{c_{B}^{2}+p_{s}^{2}}} 1+\epsilon \\
& \left.+\lim _{P \rightarrow \infty}\left[\sum_{k=0}^{P}\left[c_{B}^{2}+b(2 k+1)+\lambda_{B} b\right)^{1 / 2}-\left(c_{B}^{2}+b(2 k+1)-\lambda_{B} b\right)^{1 / 2}\right]-\lambda_{B} \int_{\frac{a^{2}}{2}}^{P b} \frac{d w}{\sqrt{\zeta_{\mathrm{ex}}^{2}+2 w}}\right]
\end{aligned}
$$

where the integral on the first line is to be evaluated in the dimensional regulation scheme and can be evaluated using (4.88).

\subsection{Matching under duality}

We have demonstrated above that the critical boson theory at zero chemical potential has a propagator, whose zeroes predict a spectrum of single particle energies at (Lorentzian)

$$
E= \pm \zeta_{ \pm}^{B}(n)
$$

where $\zeta_{ \pm}^{B}(n)$ are given by (5.21) where $c_{B}$ is required to solve the gap equation (5.25).

We now wish to compare the result (5.26) with our results for the spectrum of single particle energies in the fermionic theory of the previous section. The variable $n$ that appears in (5.26) can simply be identified with the Landau level label $\nu$ used, for instance in (2.26). Comparing (5.21) with (4.56), we see that the spectrum of bosonic and fermionic single particle energies agrees exactly provided we make the identification

$$
c_{B}^{2}=c_{F}^{2}=\zeta_{\mathrm{ex}}^{2} \pm \lambda_{F} b
$$

(where the sign \pm in (5.27) applies in the Fermionic positive/negative exceptional bands). 
In order to complete the check that the zero chemical potential bosonic single particle energy spectrum agrees with the corresponding single particle spectrum of fermionic energies, we must verify that the gap equation (5.25) reduces to the corresponding Fermionic gap equation under the variable change (5.27).

Recall that we have performed the bosonic computation in the case that $m_{B}^{\text {cri }}$ is positive (this is why were able to perform our computation without turning on a bosonic condensate). Using the relationship

$$
m_{F}=-\lambda_{B} m_{B}^{\text {cri }}
$$

it follows that we are working in the case that $\lambda_{F}$ and $m_{F}$ have the same sign. We have also performed our bosonic computation assuming that the chemical potential lies between the positive and negative Landau $\nu=0$ Landau Levels. When $\operatorname{sgn}\left(m_{F}\right)>0$ this condition puts us in the fermionic 'negative exceptional band'. On the other hand when $\operatorname{sgn}\left(m_{F}\right)<0$ this condition puts us in the fermionic positive exceptional band.

In summary, duality predicts that the bosonic gap equation should match (under the map (5.27)) with the fermionic gap equation in the negative exceptional band when $m_{F}$ and $\lambda_{F}$ are both positive, and the fermionic gap equation in the positive exceptional band when $m_{F}$ and $\lambda_{F}$ are both negative.

It is easy to verify that the prediction of the previous paragraph is indeed borne out provided we assume that $\zeta_{\mathrm{ex}}$ has the same sign as $m_{F}$ at every value of $\lambda_{F}$. Under this assumption, the conditions of the previous paragraph put us in the special case studied in section 4.10. It is easy to verify that (5.25) reduces to (4.102) (using (5.27)) when $m_{F}, \lambda_{F}$ and $\zeta_{\mathrm{ex}}$ are all positive, but reduces to (4.108) when $m_{F}, \lambda_{F}$ and $\zeta_{\mathrm{ex}}$ are all negative.

In order to complete our demonstration of the matching of gap equations under duality, we need to demonstrate that our assumption about the sign of $\zeta_{\text {ex }}$ is correct. We do not, unfortunately, have an analytic proof of this fact. The numerical evidence presented in the previous section, however, appears to back this claim.

It thus appears that all our results are in perfect agreement with the predictions of duality, although a watertight demonstration of this fact would need an analytic proof of the assumption we have made earlier in this section for the sign of $\zeta_{\mathrm{ex}}$.

\section{$6 \quad$ Future directions}

Working at leading order in the large $N$ limit, in this paper we have computed the exact (i.e. all orders in the t' Hooft coupling) fermion - fermion propagator of the Chern Simons gauged regular fermion theory in the fundamental representation. A key structural feature of this propagator - whenever we have reliably been able to compute it - is that its only non analyticities in frequency space are a set of poles. While the propagator that we have computed is not itself gauge invariant, its poles capture the physical one particle excitations of the theory. In the free limit $\lambda_{F} \rightarrow 0$ the poles of our propagator lie at the locations of Landau levels. Our exact determination of the poles of our propagator allows us to track the change in the energies of Landau levels as a function of the t'Hooft coupling. The final result for the energies of unfilled Landau Levels is satisfyingly simple; the spectrum of 
single particle energies takes the simple form one might naively have guessed for a system of free relativistic spin $s$ particles propagating in a magnetic field (see around (2.46)) with $s$ given by (4.57), the true spin of the anyonic excitations in our theory.

The large $N$ limit ensures that our propagator has only poles (and not also cuts) at all values of $\lambda_{F}$; cuts in the propagator all come from diagrams with holes or boundaries (in the sense of the t' Hooftian counting of large $N$ Feynman diagrams) and so are suppressed at large $N$. In this sense our large $N$ limit may be the nearest one can come to defining a theory of non interacting relativistic spin $s$ particles. The simple structure of the energies of unfilled Landau Levels is a further illustration of this fact.

We find our results for filled Landau levels, and especially for Landau levels in the process of being filled, particularly interesting. As we have explained in the introduction, in the interacting theory (and unlike in the free theory) the minimum chemical potential at which a Landau Level is completely filled differs from the maximum chemical potential at which the same Landau level is completely empty. The first chemical potential is the one at which the pole in the propagator - corresponding to this Landau level - lies just below our integration contour in $\omega$ (frequency) space, while the second chemical potential is the one at which the same pole lies just above the same integration contour. We call the difference between the first chemical potential and the second chemical potential the width of the corresponding Landau level. This width can be either positive or negative.

A careful analysis of the physics within the width of any Landau level may turn out to be one of the most important research directions that follow from the work presented in this paper. We pause to discuss this point at some length.

Let us first consider the case of Landau levels with a positive width. In this case the analysis of this paper yields no valid solutions to the gap equation when $\mu$ lies within the width of any particular Landau level. At the technical level this happens because a pole in the complex $\omega$ plane hits the integration contour either from below (at the top of the band) or from above (at the bottom of the band). It is tempting to obtain new solutions to the gap equations at intermediate values of $\mu$ by adopting the following prescription. We could allow the pole corresponding to a particular Landau to lie precisely on the integration contour, and then split up this pole in an ad hoc manner so that a fraction $f$ of the pole lies under the contour and the remaining fraction $1-f$ of the pole lies above the contour (the special case $f=\frac{1}{2}$ defines the principle value prescription). Intuitively this corresponds to filling up part of the Landau Level under study while leaving the rest of it unfilled. If we adopt this prescription then $\zeta_{\mathrm{ex}}^{2}$ and hence the value of $\mu$ corresponding to this situation ${ }^{36}$ will depend on $f$. As we vary $f$ from unity to zero, this prescription presumably gives us a solution to the gap equation for values of $\mu$ that lies between the lower and upper end of the Landau Level width. This (natural but so far ad hoc) prescription would give us a result for the fermion propagator within a Landau level and would potentially allow us to study its physics. The discussion of this paragraph provides a natural first guess for the nature of dynamics within a particular Landau level, one that should certainly be pursued in future work.

\footnotetext{
${ }^{36}$ This is the value of $\mu$ at which the Landau level pole of interest lies on the contour.
} 
While the prescription developed in the previous paragraph is simple and appealing it may not be completely correct. It is possible that the correct propagator (when $\mu$ lies within the width of the Landau Level) has a more complicated singularities than poles even in the strict Large $\mathrm{N}$ limit implying that fermion dynamics within the Landau band is not single particle in nature, even in the strict large $N$ limit. At the technical level a conclusion of this sort would follow if, for instance, infrared divergences within a Landau band turn out to complicate the naive large $N$ expansion, and cause naively sub-leading graphs to actually contribute at leading order in large $N$. The classic work of Sung-Sik Lee [53] provides a precedent for a similar effect in a vaguely similar context. As dynamics at chemical potentials that lie within a Landau level is of great interest - for instance for the study of the quantum hall effect — we feel that these issues deserve careful investigation in future work.

We have so far discussed the case in which a given Landau Level has a positive width. In certain ranges of parameters (though never at large enough values of $\left|\lambda_{F}\right|$ ) we find that the width of our Landau Levels are sometimes negative. At the physical level this is not particularly strange - it simply means that the energy needed to add the last fermion into an almost filled band is lower than the energy needed to add the first Fermion into the otherwise empty band. We would expect this to happen whenever the effective interaction between fermions is attractive rather than repulsive.

At the technical level, whenever we have a negative Landau level width, for every $\mu$ that lies within this width we have two solutions for the fermion propagator. This may, at first seem like a better situation to be in than the case of positive width (in which case we had no solution for the propagator at intermediate values of $\mu$ ) but we believe this is not really the case. The reason for this is that the two solutions that we do have in this case correspond to the solution for a fully filled or a completely empty Landau level. For physical reasons we are really interested in studying a partially filled Landau level. ${ }^{37}$

Hence in this case as well it is natural to seek a third solution, for every value of $\mu$ within the band, for the fermion propagator. As in the discussion above, a naive solution of this form could be generated by splitting the pole on the propagator into a part above and a part below. As in the discussion above this naive solution may - or may not capture the true nature of the propagator. This question deserves further study.

Apart from the (intensely interesting but potentially tricky) study of the dynamics within a broadened Landau band, the work in this paper should be generalized in several ways. First, it should be straightforward to use the results of this paper to compute the

\footnotetext{
${ }^{37}$ When the width of the Landau level is negative, we have already seen that there is a range of $\mu$ in which there exist atleast two saddle point solutions. For the sake of argument let us assume the solutions we already know are the dominant ones for thermodynamical purposes. As $\mu$ is gradually increased, we would expect our theory to undergo a first order phase transition from the saddle in which the level is completely empty to the saddle in which it is completely filled. The charge density will increase discontinuously across this phase trasition. At fixed intermediate values of charge (rather than fixed chemical potential, i.e. in the 'microcanonical' ensemble) the system could well undergo a phase separation ("half water, half steam") that breaks translational invariance. It would be useful to replace these speculations with quantitive results from an honest evaluation of the partition function. We hope to return to this issue soon. We thank D. Tong for related discussions.
} 
thermodynamical free energy of our system at zero temperature but arbitrary chemical potential. A concrete outcome of such a generalization would be a formula for the charge of our system as a function of the chemical potential.

Next, it may be possible, and would be interesting, to generalize the study of this paper to finite temperature.

Next, we have already partially verified that the results of this paper are consistent with the predictions of the level rank type bosonisation duality conjectured between the Chern Simnons gauged regular fermion theory and the critical boson theory. We performed this verification by directly solving the Chern Simons gauged critical boson theory at zero chemical potential and verifying that our final results for single particle free energies matches those of the fermionic computation. It would be very useful to complete this verification by performing the needed bosonic computation at finite chemical potential, perhaps using the methods of $[37,39]$.

The computations mentioned in the last paragraph are interesting in their own right for a qualitative reason. It is well known that free bosons unstable to unchecked condensation in a large enough chemical potential background. Interaction effects presumably cure this instability. It is of great physical interest to understand in detail precisely how this happens. In the results presented in this paper we have already seen signs of strange behaviour for filled Landau levels in the limit $\lambda_{B} \rightarrow 0$. In this limit the width of, for instance, the first Landau level diverges. It is very important to semi quantitatively understand this phenomenon in qualitative physical terms. We leave this for future work.

Another related issue concerns the charge of a filled Landau level. In the free fermion theory the charge density of a single filled Landau level is given by $\frac{b N_{F}}{2 \pi}$. While we have not studied this issue, it is certainly possible that the charge of a filled Landau level is not renormalized as a function of $\lambda_{F}$. If this turns out to be the case, the charge density of a filled Landau level can be rewritten as $\frac{b N_{B}\left|\lambda_{F}\right|}{2 \pi\left|\lambda_{B}\right|}$ and so translates to a charge density that diverges, in units of $N_{B}$, as $\lambda_{B}$ is taken to zero. If the (admittedly unsubstantiated) guesses of this paragraph turn out to be even qualitatively correct, they suggest that the occupation number of any particular bosonic state (in a filled Landau level) diverges as $\lambda_{B} \rightarrow 0$. It should not be difficult - and would be very interesting - to perform computations that verify this guess. It would also be interesting to reproduce this behaviour starting from the bosonic theory, and so understand it qualitatively in bosonic terms. We hope to return to this issue in future work.

Finally we find it intriguing that the leading large $N$ gap equation in the presence of a background magnetic field, for the fundamental fermion (and fundamental boson) propagators, differ from the corresponding gap equations at zero magnetic field in only two ways. First all derivatives are replaced by background gauge covariant derivatives. Second that products of the propagator and the self energy are replaced by star products. It would be interesting to investigate whether a similar replacement rule applies also for the Schwinger Dyson equations for higher (e.g 4 point) correlators of the fermions/bosons, and also whether a similar replacement rule persists also at sub-leading orders in $\frac{1}{N}$. We also leave this question to future work. 


\section{Acknowledgments}

We would like to thank A. Vishwanath for a discussion that initiated this project. We would like to thank A. Dey, K. Damle, R. Gopakumar, T. Hartman, S. Jain, L. Janagal, G. Mandal, N. Prabhakar, D. Radicevic, R. Sen Sharma, A. Strominger, G. Torraba, V. Tripathy and S. Trivedi and especially A. Gadde and N. Seiberg for useful discussions. We would also like to thank A. Dey, G. Mandal, N. Prabhakar, N. Seiberg, A. Stronminger, D. Tong, S. Wadia and A. Vishwanath for useful comments on a preliminary draft of this manuscript. The work of both authors was supported by the Infosys Endowment for the study of the Quantum Structure of Spacetime. Finally we would both like to acknowledge our debt to the steady support of the people of India for research in the basic sciences.

\section{A Conventions}

In this appendix we summarize the conventions that are used in this note

$$
\begin{aligned}
& x^{ \pm}=\frac{x^{1} \pm i x^{2}}{\sqrt{2}}, \\
& x^{ \pm}=\frac{r}{\sqrt{2}} e^{ \pm i \phi}, \quad p^{ \pm}=\frac{p^{1} \pm i p^{2}}{\sqrt{2}}, \\
& p^{ \pm}=\frac{p_{s}}{\sqrt{2}} e^{ \pm i \theta} \\
& g_{+-}=g_{-+}=g_{33}=1 \text {, } \\
& \epsilon_{123}=\epsilon^{123}=1 \text {, } \\
& \epsilon_{+-3}=-\epsilon^{+-3}=i, \\
& \epsilon_{i j}=\epsilon_{i j 3} \\
& \gamma^{1}=\sigma_{x}=\left(\begin{array}{ll}
0 & 1 \\
1 & 0
\end{array}\right), \\
& \gamma^{2}=\sigma_{y}=\left(\begin{array}{cc}
0 & -i \\
i & 0
\end{array}\right), \\
& \gamma^{3}=\sigma_{z}=\left(\begin{array}{cc}
1 & 0 \\
0 & -1
\end{array}\right) \\
& \operatorname{Tr}\left(T^{A} T^{B}\right)=\frac{1}{2} \delta_{A, B}, \quad \sum_{A}\left(T^{A}\right)_{m}^{n}\left(T^{A}\right)_{p}^{q}=\frac{1}{2} \delta_{m}^{q} \delta_{p}^{n}, \quad \gamma^{\mu} \gamma^{\nu}=i \epsilon^{\mu \nu \rho} \gamma_{\rho}+g^{\mu \nu}
\end{aligned}
$$

Conventions for Fourier transformations are

$$
f(x)=\int \frac{d^{3} P}{(2 \pi)^{3}} e^{i P \cdot x} f(P), \quad f(x, y)=\int \frac{d^{3} P}{(2 \pi)^{3}} \frac{d^{3} q}{(2 \pi)^{3}} e^{i\left(\frac{P}{2}+q\right) \cdot x+i\left(\frac{P}{2}-q\right) \cdot y} f(P, q)
$$

We will often find it useful to use following set of coordinates

$$
x_{T}=x+y, x_{R}=x-y
$$

In this new set of coordinates metric

$$
d s^{2}=d x^{2}+d y^{2}=\frac{1}{2}\left(d x_{T}^{2}+d x_{R}^{2}\right)
$$

is different by a factor and as a result of that $x_{T, R}^{ \pm}=2 x_{T, R, \mp}$. Further note that variable change from $(x, y)$ to $\left(x_{T} / 2, x_{R}\right)$ does not involve any non-trivial Jacobin. We have following map between derivatives and polynomials

$$
\begin{aligned}
i q_{\mu} f(P, q) & =\int d^{3} x d^{3} y \frac{\partial}{\partial x_{R}^{\mu}} f(x, y) e^{-i\left(\frac{P}{2}+q\right) \cdot x-i\left(\frac{P}{2}-q\right) \cdot y} \\
\frac{i P_{\mu}}{2} f(P, q) & =\int d^{3} x d^{3} y \frac{\partial}{\partial x_{T}^{\mu}} f(x, y) e^{-i\left(\frac{P}{2}+q\right) \cdot x-i\left(\frac{P}{2}-q\right) \cdot y}
\end{aligned}
$$

We will be dealing with functions of the form

$$
f(x, y)=e^{-i \frac{b}{2}\left(x^{1} y^{2}-x^{2} y^{1}\right)} f_{R}(x, y)=e^{\frac{b}{4}\left(x_{R}^{+} x_{T}^{-}-x_{R}^{-} x_{T}^{+}\right)} f_{R}(x, y)
$$


On functions of this form

$$
\begin{aligned}
& \frac{\partial}{\partial x_{R}^{-}} f\left(x_{T}, x_{R}\right)=\left(-\frac{b}{2} x_{T,-}+\frac{\partial}{\partial x_{R}^{-}}\right) f_{R}\left(x_{T}, x_{R}\right) \\
& \frac{\partial}{\partial x_{T}^{-}} f\left(x_{T}, x_{R}\right)=\left(+\frac{b}{2} x_{T,-}+\frac{\partial}{\partial x_{T}^{-}}\right) f_{R}\left(x_{T}, x_{R}\right)
\end{aligned}
$$

\section{B Constraints of gauge invariance}

In this paper we choose to work in the gauge $g_{r}$. In this gauge $\alpha(x, y)$ is rotationally invariant, i.e.,

$$
\left(x_{1} \frac{\partial}{\partial x^{2}}-x_{2} \frac{\partial}{\partial x^{1}}+y_{1} \frac{\partial}{\partial y^{2}}-y_{2} \frac{\partial}{\partial y^{1}}\right) \alpha(x, y)+\left[S_{12}, \alpha(x, y)\right]=0
$$

Where $S_{12}$ is the contribution of the spin to angular momentum. It is not present for the bosons, i.e., for bosons

$$
S_{12}=0
$$

and for fermions

$$
S_{12}=\frac{1}{4}\left[\gamma_{1}, \gamma_{2}\right]=\frac{i}{2} \gamma_{3}
$$

It also follows from (2.16) and (2.15) that

$$
e^{i \frac{b x^{1} x^{2}}{2}} \alpha(x, y) e^{-i \frac{b y^{1} y^{2}}{2}}=f_{1}\left(x_{1}-y_{1}, x_{2}, x_{3}, y_{2}, y_{3}\right)
$$

and

$$
e^{-i \frac{b x^{1} x^{2}}{2}} \alpha(x, y) e^{i \frac{b y^{1} y^{2}}{2}}=f_{2}\left(x_{1}, y_{1}, x_{2}-y_{2}, x_{3}, y_{3}\right)
$$

Eliminating $\alpha$ from above two equations are seeking consistency with infinitesimal translation in 1,2 direction solves $f_{1}, f_{2}$ upto a function that is translationally invariant in 1,2 directions. Demanding translational invariance in 3 direction as well we get

$$
\alpha(x, y)=e^{-i \frac{b}{2}\left(x^{1} y^{2}-x^{2} y^{1}\right)} \alpha_{R}(x-y)
$$

Plugging this into equation (B.1) determines rotational charge of (various components of $2 \times 2$ matrix in case of fermion) $\alpha$.

Analogous statements apply for $\Sigma(x, y)$ and we won't repeat them here.

\section{Spectrum of free particles in a magnetic field}

\section{C.1 Boson}

In this subsection we study consider the non-relativistic Schrodinger equation for a spin-less particle of mass $|M|$ in a uniform magnetic field $b$ described by Hamiltonian

$$
H=-\frac{\left(\nabla_{i}-i a_{i}\right)^{2}}{2|M|}
$$


We work in the in a rotationally invariant gauge

$$
a_{j}=-b \frac{\epsilon_{j i} x^{i}}{2}
$$

Eigen functions of the hamiltonian satisfies

$$
H \phi=E \phi
$$

We work in polar coordinates $(r, \phi)$. We seek a solution of the form

$$
\phi=\frac{e^{i l \phi}}{\sqrt{2 \pi}} R(u), \quad u=\frac{|b|}{2} r^{2}
$$

The condition that this describes a particle of spin zero determines $l=0, \pm 1, \pm 2, \ldots$ Plugging this form of $\phi$ into (C.3), it follows that $R(u)$ satisfies

$$
u R^{\prime \prime}(u)+R^{\prime}(u)+\left(-\frac{1}{4} u+\beta-\frac{m^{2}}{4 u}\right) R(u)=0
$$

where we have defined

$$
m=-\operatorname{sgn}(\mathrm{b}) 1, \quad \beta=\frac{\mathrm{E}}{\omega_{\mathrm{H}}}-\frac{\mathrm{m}}{2}, \quad \omega_{\mathrm{H}}=\frac{|\mathrm{b}|}{|\mathrm{M}|}
$$

The equation for $R$ can be transformed into confluent hypergeometric equation for $\tilde{R}$ by following change of variables

$$
R(u)=e^{-u / 2} u^{|m| / 2} \tilde{R}(u)
$$

We search for solutions that are normalisable by following inner product

$$
\left(\phi_{1}, \phi_{2}\right)=\int d^{2} x \phi_{1}(x)^{*} \phi_{2}(x)
$$

For those solutions

$$
\tilde{R}(u) \propto L_{n}^{|m|}(u)
$$

where $n=0,1,2, \ldots$ is a non-negative integer that determines energy to be

$$
E=\omega_{H}\left(n+\frac{|m|}{2}+\frac{m}{2}+\frac{1}{2}\right)
$$

Corresponding orthonormal eigen functions are given by ${ }^{38}$

$$
\phi=i^{|m|}\left[\frac{|b|}{2 \pi} \frac{n !}{(|m|+n) !}\right]^{1 / 2} e^{-i \operatorname{sgn}(\mathrm{b}) \mathrm{m} \phi} e^{-u / 2} u^{|m| / 2} L_{n}^{|m|}(u)
$$

Below we rewrite the expressions using following map

$$
m=-\operatorname{sgn}(\mathrm{b}) \mathrm{l}, \mathrm{n}=\nu-\frac{|\mathrm{l}|}{2}+\operatorname{sgn}(\mathrm{b}) \frac{1}{2}
$$

In these variables

$$
\phi=i^{|l|}\left[\frac{|b|}{2 \pi} \frac{\left(\nu-\frac{|l|}{2}+\operatorname{sgn}(\mathrm{b}) \frac{1}{2}\right) !}{\left(\nu+\frac{|l|}{2}+\operatorname{sgn}(\mathrm{b}) \frac{1}{2}\right) !}\right]^{1 / 2} e^{i l \phi} e^{-u / 2} u_{\nu-\frac{|l|}{2}+\operatorname{sgn}(\mathrm{b}) \frac{1}{2}}^{|l|} L^{|l|}(u), E=\omega_{H}\left(\nu+\frac{1}{2}\right)
$$

\footnotetext{
${ }^{38} L_{n}^{l}$ is associated Laguerre polynomial. For non-negative integer $n, L_{n}^{l}(x)$ is a polynomial in $x$ of degree $n$ normalised as $L_{n}^{l}(0)=\frac{\Gamma(n+l+1)}{\Gamma(n+1) \Gamma(l+1)}$. For negative integer $n, L_{n}^{l}(x)$ is taken to be zero by definition.
} 


\section{C.2 Fermion}

In this subsection we study spectrum of Dirac hamiltonian in presence of constant magnetic field. More precisely we study solutions of the following equation

$$
H \psi=\gamma^{3}\left(\vec{\gamma} \cdot(\vec{\partial}-i \vec{a})+m_{F}\right) \psi=E \psi
$$

Where gauge field is given by

$$
a_{j}=-b \frac{\epsilon_{j i} x^{i}}{2} .
$$

This corresponds to constant magnetic field through 12 plane. Working in polar coordinates (C.14) can be rewritten as

$$
H=H_{0}+i \gamma^{3} \vec{\gamma} \cdot \vec{e}_{r} H_{r}+i \gamma^{3} \vec{\gamma} \cdot \vec{e}_{\phi} H_{\phi}
$$

Here unit vectors are

$$
\vec{e}_{r}=\frac{\vec{r}}{r}, \quad \vec{e}_{\phi}=-\sin (\phi) \vec{e}_{1}+\cos (\phi) \vec{e}_{2}
$$

and

$$
H_{0}=\gamma^{3} m_{F}, \quad H_{r}=-i \partial_{r}, \quad H_{\phi}=-i \frac{1}{r} \partial_{\phi}-a_{\phi}
$$

At this point we make a similarity transformation $\Lambda$ so that $\vec{\gamma} \cdot \vec{e}_{r}$ and $\vec{\gamma} \cdot \vec{e}_{\phi}$ gets mapped to $\gamma^{1}$ and $\gamma^{2}$ respectively, i.e.,

$$
\Lambda \vec{\gamma} \cdot \vec{e}_{r} \Lambda^{-1}=\gamma^{1}, \quad \Lambda \vec{\gamma} \cdot \vec{e}_{\phi} \Lambda^{-1}=\gamma^{2}, \quad \Lambda=\sqrt{r} e^{\frac{i}{2} \gamma^{3} \phi}
$$

Correspondingly we have

$$
\tilde{H}=\Lambda H \Lambda^{-1}, \quad \tilde{\psi}=\Lambda \psi
$$

Angular part is easily diagonalised by

$$
\tilde{\psi}_{ \pm}=\frac{e^{i J \phi}}{\sqrt{2 \pi}} \chi(r)_{ \pm}
$$

Where \pm refers to upper and lower component of the spinor. At this point we know angular dependence of the field completely. We impose the condition that under rotation the field behaves as a fermion

$$
e^{-i \frac{1}{2} \gamma^{3} 2 \pi} \psi(r, 2 \pi)=-\psi(r, 0)
$$

This implies $j= \pm \frac{1}{2}, \pm \frac{3}{2}, \ldots$

Radial dependance of the field is determined from two first order coupled equations given below

$$
\chi_{\mp}(r)=\frac{1}{E \pm m_{F}}\left(\mp \frac{d}{d r}+\frac{J}{r}-\frac{b}{2} r\right) \chi_{ \pm}(r)
$$

Below we first consider the case of $|E| \neq\left|m_{F}\right|$ and consider this special of $|E|=\left|m_{F}\right|$ carefully later.

For $|E| \neq\left|m_{F}\right|$, above first order equations can be put in the following form (Kummer's equation) by eliminating one of the variables

$$
u \tilde{\chi}_{ \pm}^{\prime \prime}(u)+\left(1-u+\alpha_{ \pm}\right) \tilde{\chi}_{ \pm}^{\prime}(u)+n \tilde{\chi}_{ \pm}(u)=0
$$


where

$$
\alpha_{ \pm}=\left|J \mp \frac{1}{2}\right|, u=\frac{|b|}{2} r^{2}, E^{2}=m^{2}-b\left(J \pm \frac{1}{2}\right)+|b|\left(2 n+1+\alpha_{ \pm}\right)
$$

and

$$
\chi_{ \pm}(r)=u^{\alpha_{ \pm}+1 / 2} e^{-u / 2} \tilde{\chi}_{ \pm}(u)
$$

Among two linearly independent solution of (C.24), only solution that is regular at the origin and normalisable according to

$$
(\chi, \psi)=\int d^{2} x \chi^{\dagger}(\vec{x}) \psi(\vec{x})
$$

are given by

$$
\tilde{\chi}_{ \pm}(u) \propto L_{n}^{\alpha_{ \pm}}(u)
$$

Further $n$ is restricted to take values $0,1,2, \ldots$ only. Given the value of $J, E$ we choose one sign from above equation and determine the other component by pugging it into r.h.s. of (C.23).

Solution for $|E|=\left|m_{F}\right|$ are obtained by setting one of $\chi_{+}, \chi_{-}$to zero. In that case when the component that vanishes is plugged into r.h.s. of (C.23), $\operatorname{sgn}(\mathrm{E})$ is determined in terms of $\operatorname{sgn}\left(\mathrm{m}_{\mathrm{F}}\right)$ for consistency (the denominator must vanish for the other component to be non-zero).

Finally the undetermined proportionality constant is fixed by demanding orthonormality of various solutions. Below we summarize the results.

As is usual, the Dirac Hamiltonian has eigenfunctions of both positive and negative energy. We use the label $\eta$ to distinguish the two kinds of solutions; $\eta=1$ for positive energy solutions and $\eta=-1$ for negative energy solutions. Positive and negative energy solutions are both further labelled by two discrete parameters, which we call $n$ and $J$. These parameters range over the values (together with exceptional values listed below) ${ }^{39}$

$$
\begin{array}{ll}
n=1,2, \ldots \quad J= \pm \frac{1}{2}, \pm \frac{3}{2}, \pm \frac{5}{2}, \ldots \\
n=0, J= \pm \frac{1}{2}, \pm \frac{3}{2}, \pm \frac{5}{2}, \ldots \quad \text { when } b J<0 \\
n=0, J=-\frac{1}{2},-\frac{3}{2},-\frac{5}{2}, \ldots \quad \text { when } b<0
\end{array}
$$

In addition we have the exceptional range of parameters

$$
\begin{array}{lll}
n=0 & J=\frac{1}{2}, \frac{3}{2}, \frac{5}{2}, \ldots & \text { when } b>0 \text { and } \eta m_{F}>0 \\
n=-1 & J=-\frac{1}{2},-\frac{3}{2},-\frac{5}{2}, \ldots & \text { when } b<0 \text { and } \eta m_{F}<0
\end{array}
$$

As we will see below, the exceptional range of parameters correspond to states with $|E|=$ $\left|m_{F}\right|$. The quantity $J$ just labels the angular momentum of states; $J$ runs over half integer values because the total angular momentum is the sum of an orbital and a spin component.

\footnotetext{
${ }^{39}$ Note that condition on range of parameters corresponds to $n_{ \pm} \geq-1$ (defined in (C.30)) excluding the particular case of $n_{+}=n_{-}=-1$. With further restriction that $n_{+}=-1$ or $n_{-}=-1$ is allowed only if $\eta b m_{F}>0$.
} 
We denote the most general solution by $\psi^{\eta n J}$. Recall that $\psi^{\eta n J}$ is a two component spinor, whose components we denote by

$$
\psi^{\eta n J}=\left[\begin{array}{l}
\psi_{+}^{\eta n J} \\
\psi_{-}^{\eta n J}
\end{array}\right]
$$

After solving for the eigenfunctions we find following explicit results ${ }^{40}$

$$
\psi_{ \pm}^{\eta n J}(u, \phi)=c_{ \pm} \frac{e^{i \operatorname{sgn}(\mathrm{J}) l_{ \pm} \phi}}{\sqrt{2 \pi}} u^{\frac{l_{ \pm}}{2}} e^{-\frac{u}{2}} L_{n_{ \pm}}^{l_{ \pm}}(u)
$$

where $\left(u=\frac{b}{2} x_{s}^{2}, \phi\right)$ are coordinates on space and ${ }^{41}$

$$
\begin{aligned}
& l_{+}=\left|J-\frac{1}{2}\right|, l_{-}=\left|J-\frac{1}{2}\right|+\operatorname{sgn}(J), \mathrm{n}_{+}=\mathrm{n}, \mathrm{n}_{-}=\mathrm{n}-\frac{1}{2} \operatorname{sgn}(\mathrm{J})(1+\operatorname{sgn}(\mathrm{bJ})) \\
& c_{-}=c_{+}\left(\frac{\sqrt{2|b|}}{m+\operatorname{sgn}(\mathrm{J}) \mathrm{E}^{\eta \mathrm{nJ}}}\right)^{\operatorname{sgn}(\mathrm{J})} \\
& c_{+}=\left(\frac{1}{|b|}\left(\frac{\Gamma\left(1+n_{+}+l_{+}\right)}{\Gamma\left(1+n_{+}\right)}+\left(\frac{c_{-}}{c_{+}}\right)^{2} \frac{\Gamma\left(1+n_{-}+l_{-}\right)}{\Gamma\left(1+n_{-}\right)}\right)\right)^{-1 / 2}
\end{aligned}
$$

At this point we note an important property of the eigenfunctions given in (C.29) - $\psi_{ \pm}^{\eta n J}$ is non-zero at the origin only if $l_{ \pm}=0$, i.e., precisely when they don't depend on azimuthal angle $\phi .{ }^{42}$

The energy $E^{\eta n J}$ of the eigensolution $\psi^{\eta n J}$ is given by ${ }^{43}$

$$
\begin{aligned}
E^{\eta n J} & =\eta \sqrt{m_{F}^{2}+|b|\left(2 n+1+\left|\left(J-\frac{1}{2}\right)\right|-\operatorname{sgn}(\mathrm{b})\left(\mathrm{J}+\frac{1}{2}\right)\right)} \\
& =\eta \sqrt{m_{F}^{2}+|b|\left(2 n+1-\frac{1}{2} \operatorname{sgn}(\mathrm{J})(1+\operatorname{sgn}(\mathrm{bJ}))+|\mathrm{J}|(1-\operatorname{sgn}(\mathrm{bJ}))\right)}
\end{aligned}
$$

Notice that while the wave functions (C.29) depend separately on $J$ and $n$, the energy $E^{\eta n J}$ depends on these discrete parameters only through the combination

$$
2 \nu=2 n+1-\frac{1}{2} \operatorname{sgn}(J)(1+\operatorname{sgn}(\mathrm{bJ}))+|J|(1-\operatorname{sgn}(\mathrm{bJ}))
$$

The formula for the eigenenergies, (C.31), takes the following simplified form when reexpressed in terms of $\nu$

$$
E^{\eta \nu}=\eta \sqrt{m_{F}^{2}+2|b| \nu}
$$

\footnotetext{
${ }^{40} L_{n}^{l}$ is associated Laguerre polynomial. For non-negative integer $n, L_{n}^{l}(x)$ is a polynomial in $x$ of degree $n$ normalised as $L_{n}^{l}(0)=\frac{\Gamma(n+l+1)}{\Gamma(n+1) \Gamma(l+1)}$. For negative integer $n, L_{n}^{l}(x)$ is taken to be zero by definition. In the exceptional range of parameters, the expressions $(2.50)$ involve the quantity $L_{-1}^{l_{ \pm}}$. Consequently the final expressions for $\psi_{ \pm}$listed in (2.51) only involve the functions $L_{n}^{l}(x)$ for $n \geq 0$.

${ }^{41}$ Here $|x|$ stands for absolute value of $x$.

${ }^{42}$ This property ensures that wave-functions are not singular at the origin.

${ }^{43}$ Note that expression of energy given in (C.31) is invariant under following simultaneous replacements: $b \rightarrow-b, J \rightarrow-J, m_{F} \rightarrow-m_{F}, n \rightarrow n-\frac{1}{2} \operatorname{sgn}(\mathrm{J})(1+\operatorname{sgn}(\mathrm{bJ}))$. This is reminiscent of parity invariance of the theory under consideration.
} 
Below we discuss the range over which $\nu$ takes values. Let us first suppose that $b J<0$. In this case $\nu$ simplifies to

$$
2 \nu=2(n+|J|)+1 .
$$

When $b J>0$, on the other hand, $\nu$ is independent of $|J|$ and we have

$$
2 \nu=2 n+1-\operatorname{sgn}(J)
$$

Notice that in both cases (C.34) and (C.35) $\nu$ ranges over integer values. The specific range of values is as follows:

$$
\begin{array}{ll}
\nu=1,2,3 \ldots & b \eta m_{F}<0 \\
\nu=0,1,2,3 \ldots & b \eta m_{F}>0
\end{array}
$$

If we fix $\nu$ at some value - say $\nu_{0}$. Let us first take the case $b>0$. For $\nu_{0}>0$ the set of energy eigenstates with any particular value of $\nu_{0}$ (and so with eigen energy determined by $\nu_{0}$ ) is given by

$$
\left(0,-\nu_{0}+\frac{1}{2}\right), \quad\left(1,-\nu_{0}+\frac{3}{2}\right) \ldots\left(\nu_{0}-1,-\frac{1}{2}\right), \quad \sum_{l=0}^{\infty}\left(\nu_{0}, l+\frac{1}{2}\right)
$$

(where we have denoted the labels of states by the notation $(n, J)$ ). As we have remarked above, eigenstates with $\nu_{0}=0$ exist only when $\eta m_{F}>0$, in which case the set of $(n, J)$ values that lead to $\nu_{0}=0$ are given by

$$
\sum_{l=0}^{\infty}\left(\nu_{0}, l+\frac{1}{2}\right)
$$

When $b<0$, on the other hand, the set of values that lead to any value of $\nu_{0}>0$ are given by

$$
\left(0, \nu_{0}-\frac{1}{2}\right), \quad\left(1, \nu_{0}-\frac{3}{2}\right) \ldots\left(\nu_{0}-1, \frac{1}{2}\right), \quad \sum_{l=0}^{\infty}\left(\nu_{0}-1,-\left(l+\frac{1}{2}\right)\right)
$$

The particular case of $\nu_{0}=0$ is obtained when $\eta m_{F}<0$ and that corresponds to the set

$$
\sum_{l=0}^{\infty}\left(\nu_{0}-1,-\left(l+\frac{1}{2}\right)\right)
$$

\section{Star product and twisted convolution}

In this appendix we review standard properties of the Moyal star product (see, for example, [47] and references therein for more details). For two given functions $\phi_{1,2}$ on $R^{d}$, (Moyal-) star product is defined to be the following function

$$
\left(\phi_{1} \star_{\Theta} \phi_{2}\right)(q)=\lim _{p \rightarrow q} e^{\frac{i}{2} \Theta^{\mu \nu} \frac{\partial}{\partial p_{\mu}} \frac{\partial}{\partial q_{\mu}}} \phi_{1}(p) \phi_{2}(q)
$$


Where $\Theta$ is an anti-symmetric non-degenerate 2 form. Another equivalent definition is given by

$$
\left(\phi_{1} \star_{\Theta} \phi_{2}\right)(q)=\int d^{d} q_{1} d^{d} q_{2} \phi_{1}\left(q_{1}\right) \phi_{2}\left(q_{2}\right) K\left(q_{1}, q_{2}, q\right)
$$

where

$$
K\left(q_{1}, q_{2}, q\right)=\frac{1}{\operatorname{det}(\Theta)} e^{2 i\left(\Theta^{-1}\right)^{\mu \nu}\left(q-q_{1}\right)_{\mu}\left(q-q_{2}\right)_{\nu}}
$$

It follows from the definition that, star product is not commutative. In Fourier space star product becomes twisted convolution

$$
\int \frac{d^{d} q}{(2 \pi)^{d}}\left(\phi_{1} \star_{\Theta} \phi_{2}\right)(q) e^{i x q}=\int d^{d} y \phi_{1}(x-y) \phi_{2}(y) e^{-\frac{i}{2} \Theta_{\mu \nu}(x-y)^{\mu} y^{\nu}} \equiv\left(\phi_{1} *_{\Theta} \phi_{2}\right)(x)
$$

The Moyal star product is isomorphic to product of operators acting on the Hilbert space of square integrable wave functions in one dimension (i.e. the Hilbert space relevant to the quantum mechanics for a non relativistic particle on a line). We pause to describe this isomorphism.

If we define anti-commuting operators by demanding

$$
\left[\hat{q}_{\mu}, \hat{q}_{\nu}\right]=i \Theta_{\mu \nu}
$$

and with any function $f$ of $q_{\mu}$ we associated a Weyl ordered operator $\hat{f}$ by $y^{44}$

$$
f(x)=\int \frac{d^{d} q}{(2 \pi)^{3}} e^{i q \cdot x} f(q), \quad \hat{f}(\hat{q})=\int d^{d} x e^{-i \hat{q} \cdot x} f(x)
$$

Then these operators enjoy the following identity

$$
\hat{f}(\hat{q}) \hat{g}(\hat{q})=\hat{h}(\hat{q}), \quad\left(f \star_{\Theta} g\right)(q)=h(q)
$$

With the help of above theorem, we can convert star products to operator products and vice-versa.

Comparing (D.4) with (3.1) we see that for the purpose of this paper we have

$$
\Theta_{\mu \nu}=b \epsilon_{\mu \nu 3}
$$

\section{D.1 Star product basis}

In this subsection we follow [47] to construct a basis of functions on $R^{2}$ which has simple behaviour under star product (twisted convolution). To this end we restrict our attention to $b>0$ and define ${ }^{45}$

$$
\hat{a}=\frac{\hat{q}^{+}}{\sqrt{b}}, \quad \hat{a}^{\dagger}=\frac{\hat{q}^{-}}{\sqrt{b}} \Longrightarrow\left[\hat{a}, \hat{a}^{\dagger}\right]=1
$$

\footnotetext{
${ }^{44}$ Here we denote functions in position and momentum space both by same symbol, but they are in general different mathematical functions in strict sense.

${ }^{45}$ Note that in any dimension we can always put $\Theta$ into canonical form to define set of such creation, annihilation operators.
} 
In terms of these operators we consider following basis of operators ${ }^{46}$

$$
\hat{e}_{m, n}(\hat{q}) \equiv|m\rangle\langle n|=: \frac{\left(\hat{a}^{\dagger}\right)^{m}}{\sqrt{m !}} e^{-\hat{a}^{\dagger} \hat{a}} \frac{(\hat{a})^{n}}{\sqrt{n !}}:, \quad n, m=0,1,2, \ldots
$$

These define corresponding functions in position space by

$$
\hat{e}_{m, n}(\hat{q})=\int d^{2} x e^{-i \hat{q} \cdot x} e_{m, n}(x)
$$

More explicitly these functions are given by

$$
e_{m, n}(u, \phi)=i^{m-n} \frac{b}{2 \pi}\left(\frac{n !}{m !}\right)^{1 / 2} e^{-i(m-n) \phi} u^{\frac{m-n}{2}} e^{-\frac{u}{2}} L_{n}^{m-n}(u)
$$

where $u=\frac{b}{2} r^{2},(r, \phi)$ are polar coordinates on space. The algebra of these operators turns into identities

$$
\begin{aligned}
\left(e_{n, p} *_{b} \quad e_{p^{\prime}, m}\right)(\vec{x}) & =\delta_{p p^{\prime}} e_{n, m}(\vec{x}) \\
\sum_{n=0}^{\infty} e_{n, n}(\vec{x}) & =\delta(\vec{x})
\end{aligned}
$$

Further the functions $e_{n, m}$ are orthogonal to each other in the usual quantum mechanical sense

$$
\int d^{2} x \frac{2 \pi}{b} e_{n, m}^{*}(\vec{x}) e_{n^{\prime}, m^{\prime}}(\vec{x})=\delta_{n n^{\prime}} \delta_{m m^{\prime}}
$$

\section{D.2 Expanding functions in star product basis}

In addition to (D.13), in this paper we will need to evaluate the integral of $e_{m, n}$ and $e_{m^{\prime}, n^{\prime}}^{*}$ wighted by $1 / x^{+}$(the last factor comes from the Chern Simons gauge boson propagator in lightcone gauge). The relevant integrals can be done by using the explicit expressions in (D.11), first performing all angular integrals and then doing the radial integrals. At this stage the integrand has a product of associated Laguerre polynomials, and the needed integrals can be obtained by manipulating the integrand into a standard form (i.e. a form whose integrals is presented in tables of Integrals) using recursion relations and other simple identities of associated Laguerre Polynomials. In this brief subsection we only present our final results ${ }^{47}$

$$
\begin{aligned}
\int d^{2} x \frac{2 \pi}{b} e_{m^{\prime}, n^{\prime}}(\vec{x})^{*} e_{m, n}(\vec{x}) \frac{i}{\sqrt{b} x^{+}}= & \delta_{\left(m^{\prime}-n^{\prime}\right)-(m-n), 1} \sqrt{\frac{m ! n^{\prime} !}{n ! m^{\prime} !}} \\
& \times \begin{cases}+U\left(n^{\prime}-n\right) & \text { For } m \geq n \\
-U\left(n-\left(n^{\prime}+1\right)\right) & \text { For } m<n\end{cases}
\end{aligned}
$$

where $U(m)$ is the unit step function, i.e.

$$
U(m)=1 \quad m \geq 0, \quad U(m)=0, \quad \text { otherwise }
$$

\footnotetext{
${ }^{46}$ Here we are using standard normal ordering of $a, a^{\dagger}$,i.e., $a^{\dagger}$ on the left of $a$.

${ }^{47}$ Even though the integrand on the l.h.s. of (D.14) is singular at $|x|=0$, the singularity is integrable and so the integral on the l.h.s. of (D.14) is well defined.
} 
(The $\delta$ function on r.h.s. of (D.14) enforces momentum conservation). It follows from (D.14), (D.13) that

$$
\frac{i}{\sqrt{b}} \frac{e_{m, n}(\vec{x})}{x^{+}}= \begin{cases}+\sum_{p=0}^{\infty} \sqrt{\frac{m !(n+p) !}{n !(m+p+1) !}} e_{m+p+1, n+p}(\vec{x}) & \text { For } m \geq n \\ -\sum_{p=1}^{m+1} \sqrt{\frac{m !(n-p) !}{n !(m-p+1) !}} e_{m-p+1, n-p}(\vec{x}) & \text { For } m<n\end{cases}
$$

\section{E Fermionic gap equations}

In this appendix we reproduce the derivation of the gap equations of the Fermionic theory. The analysis of this section closely follows the work out presented in [26].

Consider the action given in (4.2) and work in momentum space. We work in the gauge $A_{-}=0$. In this gauge self-interaction of gauge field $A$ becomes trivial and action for remaining components of $A$ becomes quadratic. Using equations of motion of $A_{+}, A_{3}$ can easily be solved in terms of fermionic fields

$$
A_{3}^{C}(P)=4 \pi \lambda_{F} \frac{1}{P_{-}} \int \frac{d^{3} q}{(2 \pi)^{3}} \frac{1}{N} \bar{\psi}\left(-q+\frac{P}{2}\right) \gamma^{+} T^{C} \psi\left(q+\frac{P}{2}\right)
$$

Putting it back in the action we get an effective (purely imaginary) non-local four-fermion interaction

$$
\begin{aligned}
S= & \int \frac{d^{3} q}{(2 \pi)^{3}} \frac{d^{3} P}{(2 \pi)^{3}} \bar{\psi}\left(-q+\frac{P}{2}\right)\left(\left(i q_{\mu} \gamma^{\mu}+m_{F}\right)(2 \pi)^{3} \delta^{3}(P)-i \gamma^{\mu} a_{\mu}(-P)\right) \psi\left(q+\frac{P}{2}\right) \\
& -N 2 \pi i \lambda_{F} \int \frac{d^{3} q_{1}}{(2 \pi)^{3}} \frac{d^{3} q_{2}}{(2 \pi)^{3}} \frac{d^{3} P}{(2 \pi)^{3}} \frac{1}{\left(q_{1}-q_{2}\right)_{-}} \alpha\left(P, q_{1}\right) \gamma^{+} \alpha\left(-P, q_{2}\right) \gamma^{3}
\end{aligned}
$$

Here we have introduced fermion bilinear

$$
\alpha(P, q)=\frac{1}{N} \bar{\psi}\left(-q+\frac{P}{2}\right) \psi\left(q+\frac{P}{2}\right)
$$

where gauge indices are contracted.

To proceed further we use following standard trick

$$
\begin{aligned}
1 & =\int d \alpha \delta\left(\alpha(P, q)-\frac{1}{N} \bar{\psi}\left(-q+\frac{P}{2}\right) \psi\left(q+\frac{P}{2}\right)\right) \\
& =\int d \alpha d \Sigma e^{N \int \frac{d^{3} q}{(2 \pi)^{3}}\left(\frac{d^{3} P}{(2 \pi)^{3}} \Sigma(-P, q)\left(\alpha(P, q)-\frac{1}{N} \bar{\psi}\left(-q+\frac{P}{2}\right) \psi\left(q+\frac{P}{2}\right)\right)\right.}
\end{aligned}
$$

Plugging it in the path integration we can integrate out $\psi, \bar{\psi}$ to give an effective action for $\alpha, \Sigma$

$$
\begin{aligned}
S_{\mathrm{eff}}= & -N \int \frac{d^{3} q}{(2 \pi)^{3}} \frac{d^{3} P}{(2 \pi)^{3}} \log \operatorname{det}\left(\left(i q_{\mu} \gamma^{\mu}+m_{F}\right)(2 \pi)^{3} \delta^{3}(P)-i \gamma^{\mu} a_{\mu}(-P)+\Sigma(-P, q)\right) \\
& -N 2 \pi i \lambda_{F} \int \frac{d^{3} q_{1}}{(2 \pi)^{3}} \frac{d^{3} q_{2}}{(2 \pi)^{3}} \frac{d^{3} P}{(2 \pi)^{3}} \frac{1}{\left(q_{1}-q_{2}\right)_{-}} \alpha\left(P, q_{1}\right) \gamma^{+} \alpha\left(-P, q_{2}\right) \gamma^{3} \\
& -N \int \frac{d^{3} q}{(2 \pi)^{3}} \frac{d^{3} P}{(2 \pi)^{3}} \alpha(P, q) \Sigma(-P, q)
\end{aligned}
$$


Varying above action with respect to $\Sigma$ we get

$$
\left(\left(i q_{\mu} \gamma^{\mu}+m_{F}\right)(2 \pi)^{3} \delta^{3}(P)-i \gamma^{\mu} a_{\mu}(-P)+\Sigma(-P, q)\right) \alpha(P, q)=-(2 \pi)^{3} \delta^{3}(P)
$$

Varying above action with respect to $\alpha$ we get

$$
\Sigma(P, q)=-2 \pi i \lambda_{F} \int \frac{d^{3} q^{\prime}}{(2 \pi)^{3}} \frac{1}{\left(q-q^{\prime}\right)_{-}}\left(\gamma^{+} \alpha\left(P, q^{\prime}\right) \gamma^{3}-\gamma^{3} \alpha\left(P, q^{\prime}\right) \gamma^{+}\right)
$$

These two equations when translated to position space becomes (4.5) as presented in main text.

\section{F Details of contour integrations}

\section{F.1 The first integral}

Let us consider the integral (4.35). If the contour of integration lies either above or below both poles in the integrand on the r.h.s. of (4.35) then the integral clearly vanishes. ${ }^{48} \mathrm{In}$ general the integral evaluates to

$$
I_{1}=i \sqrt{b(n+1)} \frac{\Theta\left(\tilde{\zeta}_{+}^{F}(n)\right) \Theta\left(\tilde{\zeta}_{-}^{F}(n)\right)-\Theta\left(-\tilde{\zeta}_{+}^{F}(n)\right) \Theta\left(-\tilde{\zeta}_{-}^{F}(n)\right)}{\tilde{\zeta}_{+}^{F}(n)+\tilde{\zeta}_{-}^{F}(n)}
$$

For convenience we defined

$$
\tilde{\zeta}_{ \pm}^{F}=\zeta_{ \pm}^{F} \mp \mu
$$

It follows that if $\zeta_{+}^{F}(n)>-\zeta_{-}^{F}(n)$ (as we expect to be the case on physical grounds) and the contour passes between the two poles then

$$
I_{1}=i \sqrt{b(n+1)} \frac{1}{\zeta_{+}^{F}(n)+\zeta_{-}^{F}(n)}
$$

\section{F.2 The second integral}

When the integration contour lies either above or below all four poles in the integrand of (4.36), once again the integral $I_{2}$ vanishes. In general the integral evaluates to

$$
\begin{aligned}
I_{2}= & \frac{1}{2}\left(\frac{1}{2} \frac{\tilde{\zeta}_{+}^{F}(n)-\tilde{\zeta}_{-}^{F}(n)}{\left|\tilde{\zeta}_{+}^{F}(n)\right|+\left|\tilde{\zeta}_{-}^{F}(n)\right|}-\frac{1}{2} \frac{\tilde{\zeta}_{+}^{F}(n-1)-\tilde{\zeta}_{-}^{F}(n-1)}{\left|\tilde{\zeta}_{+}^{F}(n-1)\right|+\left|\tilde{\zeta}_{-}^{F}(n-1)\right|}\right) \\
& -\frac{1}{2}\left(\left(K_{R, I, n+1}-\mu\right) \frac{\Theta\left(\tilde{\zeta}_{+}^{F}(n)\right) \Theta\left(\tilde{\zeta}_{-}^{F}(n)\right)-\Theta\left(-\tilde{\zeta}_{+}^{F}(n)\right) \Theta\left(-\tilde{\zeta}_{-}^{F}(n)\right)}{\tilde{\zeta}_{+}^{F}(n)+\tilde{\zeta}_{-}^{F}(n)}\right) \\
& -\frac{1}{2}\left(\left(K_{R, I, n-1}+\mu\right) \frac{\Theta\left(\tilde{\zeta}_{+}^{F}(n-1)\right) \Theta\left(\tilde{\zeta}_{-}^{F}(n-1)\right)-\Theta\left(-\tilde{\zeta}_{+}^{F}(n-1)\right) \Theta\left(-\tilde{\zeta}_{-}^{F}(n-1)\right)}{\tilde{\zeta}_{+}^{F}(n-1)+\tilde{\zeta}_{-}^{F}(n-1)}\right)
\end{aligned}
$$

In order to make the discussion of this subsection concrete, let us make the reasonable assumption that

$$
-\zeta_{-}^{F}(n)<-\zeta_{-}^{F}(n-1)<\zeta_{+}^{F}(n-1)<\zeta_{+}^{F}(n)
$$

\footnotetext{
${ }^{48}$ In this case we can deform the contour to an arc at infinity. As the integrand decays like $\frac{1}{\omega^{2}}$, the contribution from this arc vanishes.
} 
Under the assumption (F.5) the conclusion of this paragraph can be restated as follows: the integral on the r.h.s. of (4.36) vanishes provided when, on the contour of integration $\operatorname{Im}(\omega)>\zeta_{+}(n)$ or $\operatorname{Im}(\omega)<-\zeta_{-}(n)$.

More generally, always assuming (F.5) we have

$$
I_{2}= \begin{cases}0 & \mu>\zeta_{+}^{F}(n) \\ A & \zeta_{+}^{F}(n-1)<\mu<\zeta_{+}^{F}(n) \\ B & -\zeta_{-}^{F}(n-1)<\mu<\zeta_{+}^{F}(n-1) \\ C & -\zeta_{-}^{F}(n)<\mu<-\zeta_{-}^{F}(n-1) \\ 0 & \mu<-\zeta_{-}^{F}(n)\end{cases}
$$

where $A, B, C$ are given by

$$
\begin{aligned}
A= & \frac{1}{2}\left(\frac{1}{2} \frac{\zeta_{+}^{F}(n)-\zeta_{-}^{F}(n)}{\zeta_{+}^{F}(n)+\zeta_{-}^{F}(n)}+\frac{1}{2}\right)-\frac{1}{2}\left(\frac{K_{R, I, n+1}}{\zeta_{+}^{F}(n)+\zeta_{-}^{F}(n)}\right) \\
B= & \frac{1}{2}\left(\frac{1}{2} \frac{\zeta_{+}^{F}(n)-\zeta_{-}^{F}(n)}{\zeta_{+}^{F}(n)+\zeta_{-}^{F}(n)}-\frac{1}{2} \frac{\zeta_{+}^{F}(n-1)-\zeta_{-}^{F}(n-1)}{\zeta_{+}^{F}(n-1)+\zeta_{-}^{F}(n-1)}\right) \\
& -\frac{1}{2}\left(\frac{K_{R, I, n+1}}{\zeta_{+}^{F}(n)+\zeta_{-}^{F}(n)}\right)-\frac{1}{2}\left(\frac{K_{R, I, n-1}}{\zeta_{+}^{F}(n-1)+\zeta_{-}^{F}(n-1)}\right) \\
C= & \frac{1}{2}\left(\frac{1}{2} \frac{\zeta_{+}^{F}(n)-\zeta_{-}^{F}(n)}{\zeta_{+}^{F}(n)+\zeta_{-}^{F}(n)}-\frac{1}{2}\right)-\frac{1}{2}\left(\frac{K_{R, I, n+1}}{\zeta_{+}^{F}(n)+\zeta_{-}^{F}(n)}\right)
\end{aligned}
$$

\section{F.3 The third integral}

In general the integral evaluates to

$$
\begin{aligned}
\tilde{I}_{2}= & \frac{1}{2}\left(\frac{1}{2} \frac{\tilde{\zeta}_{+}^{F}(0)-\tilde{\zeta}_{-}^{F}(0)}{\left|\tilde{\zeta}_{+}^{F}(0)\right|+\left|\tilde{\zeta}_{-}^{F}(0)\right|}-\frac{1}{2} \operatorname{sgn}\left(\zeta_{\mathrm{ex}}-\mu\right)\right) \\
& -\frac{1}{2}\left(\left(K_{R, I, 1}-\mu\right) \frac{\Theta\left(\tilde{\zeta}_{+}^{F}(0)\right) \Theta\left(\tilde{\zeta}_{-}^{F}(0)\right)-\Theta\left(-\tilde{\zeta}_{+}^{F}(0)\right) \Theta\left(-\tilde{\zeta}_{-}^{F}(0)\right)}{\tilde{\zeta}_{+}^{F}(0)+\tilde{\zeta}_{-}^{F}(0)}\right)
\end{aligned}
$$

Working under the reasonable assumption

$$
-\zeta_{-}^{F}(0)<\zeta_{\mathrm{ex}}<\zeta_{+}^{F}(0)
$$

we find

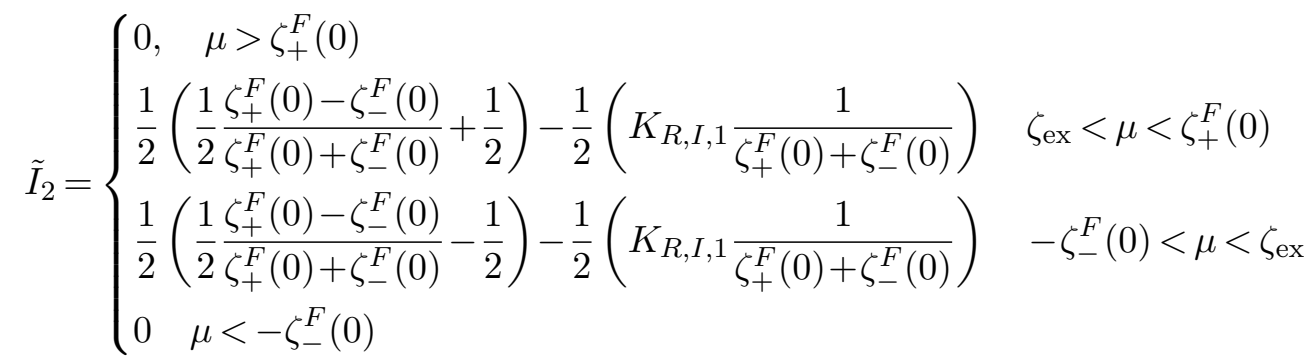




\section{G Details of fermionic recursion relations}

In this appendix, we consider fermionic gap equations for chemical potential in positive or negative exceptional band. We will show that the quantity

$$
I_{n}=\lambda_{F}^{2}\left(\sqrt{2 b} \chi_{n}\right)^{2}+\frac{1}{\left(\sqrt{2 b} \chi_{n}\right)^{2}}
$$

satisfies, for $n \geq 1$

$$
I_{n}=I_{n-1}+4
$$

To see this we simplify the following quantity by writing it entirely in terms of differences. ${ }^{49}$

$$
\begin{aligned}
\frac{1}{\chi_{n}^{2}}-\frac{1}{\chi_{n-1}^{2}}= & \left(\zeta_{+}^{F}(n)+\zeta_{-}^{F}(n)\right)^{2}-\left(\zeta_{+}^{F}(n-1)+\zeta_{-}^{F}(n-1)\right)^{2} \\
= & \left(\left(K_{R, I, n+1}+K_{R, I, n}\right)^{2}-8 K_{R,+, n+1} K_{R,-, n}\right) \\
& -\left(\left(K_{R, I, n}+K_{R, I, n-1}\right)^{2}-8 K_{R,+, n} K_{R,-, n-1}\right) \\
= & \left(K_{R, I, n+1}+K_{R, I, n-1}+2 K_{R, I, n}\right)\left(K_{R, I, n+1}-K_{R, I, n-1}\right) \\
& -8 i \sqrt{b}\left(\sqrt{n+1}\left(i \sqrt{b} \sqrt{n+1}+\Sigma_{R,+, n+1}\right)-\sqrt{n}\left(i \sqrt{b} \sqrt{n}+\Sigma_{R,+, n}\right)\right) \\
= & \left(\left(K_{R, I, n+1}-K_{R, I, n}\right)-\left(K_{R, I, n}-K_{R, I, n-1}\right)+4 K_{R, I, n}\right) \\
& \left(\left(K_{R, I, n+1}-K_{R, I, n}\right)+\left(K_{R, I, n}-K_{R, I, n-1}\right)\right) \\
& -8 i \sqrt{b}\left(\sqrt{n+1}\left(i \sqrt{b} \sqrt{n+1}+\Sigma_{R,+, n+1}\right)-\sqrt{n}\left(i \sqrt{b} \sqrt{n}+\Sigma_{R,+, n}\right)\right) \\
= & \left(\left(\Sigma_{R, I, n+1}-\Sigma_{R, I, n}\right)-\left(\Sigma_{R, I, n}-\Sigma_{R, I, n-1}\right)+4 K_{R, I, n}\right) \\
& \left(\left(\Sigma_{R, I, n+1}-\Sigma_{R, I, n}\right)+\left(\Sigma_{R, I, n}-\Sigma_{R, I, n-1}\right)\right) \\
& -8 i \sqrt{b}\left(i \sqrt{b}+\sqrt{n+1} \Sigma_{R,+, n+1}-\sqrt{n} \Sigma_{R,+, n}\right)
\end{aligned}
$$

Now we use (4.46) to replace differences in terms of $\Sigma_{R, I}$ and $\chi$. Next goal in algebraic manipulations below is to show that explicit $\Sigma_{R, I}$ dependences cancel each other, leaving behind a recursion relation involving $\chi$ alone.

$$
\begin{aligned}
\frac{1}{\chi_{n}^{2}}-\frac{1}{\chi_{n-1}^{2}}= & \left(2 \lambda_{F} b\left(\chi_{n}-\chi_{n-1}\right)+4 K_{R, I, n}\right)\left(2 \lambda_{F} b\left(\chi_{n}+\chi_{n-1}\right)\right)+8 b \\
& -8 b \lambda_{F}\left(\left(K_{R, I, n-1} \chi_{n-1}+K_{R, I, n+1} \chi_{n}\right)-\lambda_{F}\left(\left(\sqrt{b} \chi_{n}\right)^{2}-\left(\sqrt{b} \chi_{n-1}\right)^{2}\right)\right) \\
= & \left(2 \lambda_{F} b\left(\chi_{n}-\chi_{n-1}\right)+4 K_{R, I, n}\right)\left(2 \lambda_{F} b\left(\chi_{n}+\chi_{n-1}\right)\right)+8 b \\
& -8 b \lambda_{F}\left(\left(K_{R, I, n-1}-K_{R, I, n}+K_{R, I, n}\right) \chi_{n-1}\right. \\
& \left.\quad+\left(K_{R, I, n+1}-K_{R, I, n}+K_{R, I, n}\right) \chi_{n}\right)+8 b \lambda_{F}^{2}\left(\left(\sqrt{b} \chi_{n}\right)^{2}-\left(\sqrt{b} \chi_{n-1}\right)^{2}\right) \\
= & \left(2 \lambda_{F} b\left(\chi_{n}-\chi_{n-1}\right)+4 K_{R, I, n}\right)\left(2 \lambda_{F} b\left(\chi_{n}+\chi_{n-1}\right)\right)+8 b \\
& -8 b \lambda_{F}\left(\left(-2 \lambda_{F} b \chi_{n-1}+K_{R, I, n}\right) \chi_{n-1}+\left(2 \lambda_{F} b \chi_{n}+K_{R, I, n}\right) \chi_{n}\right) \\
& +8 b \lambda_{F}^{2}\left(\left(\sqrt{b} \chi_{n}\right)^{2}-\left(\sqrt{b} \chi_{n-1}\right)^{2}\right) \\
= & \left(2 \lambda_{F} b\right)^{2}\left(\chi_{n}^{2}-\chi_{n-1}^{2}\right)-4\left(2 \lambda_{F} b\right)^{2}\left(\chi_{n}^{2}-\chi_{n-1}^{2}\right)+2\left(2 \lambda_{F} b\right)^{2}\left(\chi_{n}^{2}-\chi_{n-1}^{2}\right)+8 b \\
= & -\left(2 \lambda_{F} b\right)^{2}\left(\chi_{n}^{2}-\chi_{n-1}^{2}\right)+8 b
\end{aligned}
$$

\footnotetext{
${ }^{49}$ We have used the fact that $K_{R, I, n}^{F}$ is independent of $n$.
} 
This can be rewritten as

$$
I_{n}=I_{n-1}+4
$$

\section{H Bosonic gap equations}

We consider the action given in (5.1) and work in momentum space in gauge $A_{-}=0$. Once again our work out closely follows the methodology laid out in [26]. For simplicity we set background field to zero, putting it back is trivial modification to what is derived below. Action in this gauge becomes

$$
\begin{aligned}
S_{B}= & \int \frac{d^{3} p}{(2 \pi)^{3}}\left(\frac{\kappa_{B}}{4 \pi} A_{+}^{C}(-p) i p_{-} A_{3}^{C}(p)+\bar{\phi}(-p)\left(p^{2}+M_{B}^{2}\right) \phi(p)\right) \\
& +\int \frac{d^{3} p}{(2 \pi)^{3}}\left(A_{+}^{C}(-p) J_{-}^{C}(p)+A_{3}^{C}(-p) J_{3}^{C}(p)\right) \\
& +\int \frac{d^{3} q_{1}}{(2 \pi)^{3}} \frac{d^{3} q_{2}}{(2 \pi)^{3}} \frac{d^{3} q}{(2 \pi)^{3}}\left(A_{3}^{C}\left(q_{1}\right) A_{3}^{D}\left(q_{2}\right) \bar{\phi}\left(-\frac{q_{1}+q_{2}}{2}-q\right) T^{C} T^{D} \phi\left(-\frac{q_{1}+q_{2}}{2}+q\right)\right) \\
& +4 \pi b_{4} \lambda_{B} N \int \frac{d^{3} q_{1}}{(2 \pi)^{3}} \frac{d^{3} q_{2}}{(2 \pi)^{3}} \frac{d^{3} P}{(2 \pi)^{3}} \chi\left(P, q_{1}\right) \chi\left(-P, q_{2}\right) \\
& +4 \pi^{2} x_{6} \lambda_{B}^{2} N \int \frac{d^{3} q_{1}}{(2 \pi)^{3}} \frac{d^{3} q_{2}}{(2 \pi)^{3}} \frac{d^{3} q_{3}}{(2 \pi)^{3}} \frac{d^{3} P_{1}}{(2 \pi)^{3}} \frac{d^{3} P_{2}}{(2 \pi)^{3}} \chi\left(P_{1}, q_{1}\right) \chi\left(P_{2}, q_{2}\right) \chi\left(-P_{1}-P_{2}, q_{3}\right)
\end{aligned}
$$

where we have defined

$$
\begin{aligned}
& J_{\mu}^{C}(P)=-\int \frac{d^{3} q}{(2 \pi)^{3}} \bar{\phi}\left(\frac{P}{2}-q\right) T^{C} \phi\left(\frac{P}{2}+q\right) 2 q_{\mu} \\
& \chi(P, q)=\frac{1}{N} \bar{\phi}\left(\frac{P}{2}-q\right) \phi\left(\frac{P}{2}+q\right)
\end{aligned}
$$

$A_{+}^{C}(-p)$ equation of motion from the action above is

$$
\frac{\kappa_{B}}{4 \pi} i p_{-} A_{3}^{C}(p)=-J_{-}^{C}(p)
$$

Since $A_{+}$appears only linearly in action (H.4) integrating it out sets its coefficient to zero. Only left over part of gauge field is $A_{3}$ which can be solved from (H.3) in terms of $J_{-}$. Plugging back $A_{3}$ into action (H.4) we get

$$
\begin{aligned}
& S_{B}=\int \frac{d^{3} p}{(2 \pi)^{3}}\left(\bar{\phi}(-p)\left(p^{2}+m_{B}^{2}\right) \phi(p)\right) \\
& +N \int \frac{d^{3} q_{1}}{(2 \pi)^{3}} \frac{d^{3} q_{2}}{(2 \pi)^{3}} \frac{d^{3} P}{(2 \pi)^{3}} C_{1}\left(P, q_{1}, q_{2}\right) \chi\left(P, q_{1}\right) \chi\left(-P, q_{2}\right) \\
& +N \int \frac{d^{3} q_{1}}{(2 \pi)^{3}} \frac{d^{3} q_{2}}{(2 \pi)^{3}} \frac{d^{3} q_{3}}{(2 \pi)^{3}} \frac{d^{3} P_{1}}{(2 \pi)^{3}} \frac{d^{3} P_{2}}{(2 \pi)^{3}} C_{2}\left(P_{1}, P_{2}, q_{1}, q_{2}, q_{3}\right) \chi\left(P_{1}, q_{1}\right) \chi\left(P_{2}, q_{2}\right) \chi\left(-P_{1}-P_{2}, q_{3}\right)
\end{aligned}
$$


Where $^{50}$

$$
\begin{gathered}
C_{1}\left(P, q_{1}, q_{2}\right)=4 \pi b_{4} \lambda_{B}-i 2 \pi \lambda_{B} \frac{\left(q_{1}+q_{2}\right)_{-} P_{3}-P_{-}\left(q_{1}+q_{2}\right)_{3}}{\left(q_{1}-q_{2}\right)_{-}} \\
C_{2}\left(P_{1}, P_{2}, q_{1}, q_{2}, q_{3}\right)=4 \pi^{2} x_{6} \lambda_{B}^{2}+4 \pi^{2} \lambda_{B}^{2} \frac{\left(P_{1}-P_{2}+2\left(q_{1}+q_{2}\right)\right)_{-}\left(P_{1}+2 P_{2}+2\left(q_{3}+q_{2}\right)\right)_{-}}{\left(P_{1}+P_{2}+2\left(q_{1}-q_{2}\right)\right)_{-}\left(P_{1}+2\left(q_{3}-q_{2}\right)\right)_{-}}
\end{gathered}
$$

To proceed further we use following standard trick

$$
\begin{aligned}
1 & =\int d \alpha \delta\left(\alpha(P, q)-\frac{1}{N} \bar{\phi}\left(-q+\frac{P}{2}\right) \phi\left(q+\frac{P}{2}\right)\right) \\
& =\int d \alpha d \Sigma e^{N \int \frac{d^{3} q}{(2 \pi)^{3}} \frac{d^{3} P}{(2 \pi)^{3}} \Sigma(-P, q)\left(\alpha(P, q)-\frac{1}{N} \bar{\phi}\left(-q+\frac{P}{2}\right) \phi\left(q+\frac{P}{2}\right)\right)}
\end{aligned}
$$

Plugging it in the path integration we can integrate out $\phi, \bar{\phi}$ to give an effective action for $\alpha, \Sigma$

$$
\begin{aligned}
& S_{B}=N \int \frac{d^{3} P}{(2 \pi)^{3}} \frac{d^{3} q}{(2 \pi)^{3}} \log \operatorname{det}\left(\left(q^{2}+m_{B}^{2}\right)(2 \pi)^{3} \delta^{3}(P)+\Sigma(-P, q)\right) \\
& +N \int \frac{d^{3} q_{1}}{(2 \pi)^{3}} \frac{d^{3} q_{2}}{(2 \pi)^{3}} \frac{d^{3} P}{(2 \pi)^{3}} C_{1}\left(P, q_{1}, q_{2}\right) \alpha\left(P, q_{1}\right) \alpha\left(-P, q_{2}\right) \\
& +N \int \frac{d^{3} q_{1}}{(2 \pi)^{3}} \frac{d^{3} q_{2}}{(2 \pi)^{3}} \frac{d^{3} q_{3}}{(2 \pi)^{3}} \frac{d^{3} P_{1}}{(2 \pi)^{3}} \frac{d^{3} P_{2}}{(2 \pi)^{3}} C_{2}\left(P_{1}, P_{2}, q_{1}, q_{2}, q_{3}\right) \alpha\left(P_{1}, q_{1}\right) \alpha\left(P_{2}, q_{2}\right) \alpha\left(-P_{1}-P_{2}, q_{3}\right) \\
& -N \int \frac{d^{3} q}{(2 \pi)^{3}} \frac{d^{3} P}{(2 \pi)^{3}} \alpha(P, q) \Sigma(-P, q)
\end{aligned}
$$

Varying above action with respect to $\Sigma$ we get

$$
\left(\left(q^{2}+m_{B}^{2}\right)(2 \pi)^{3} \delta^{3}(P)+\Sigma(-P, q)\right) \alpha(P, q)=(2 \pi)^{3} \delta^{3}(P)
$$

Varying above action with respect to $\alpha$ we get

$$
\begin{aligned}
& \Sigma(P, q)= \int \frac{d^{3} q^{\prime}}{(2 \pi)^{3}}\left(C_{1}\left(P, q^{\prime}, q\right)+\right. \\
&+\int \frac{d^{3} q_{1}}{(2 \pi)^{3}} \frac{d^{3} q_{2}}{(2 \pi)^{3}} \frac{d^{3} P^{\prime}}{(2 \pi)^{3}}\left(C_{2}\left(-P, q^{\prime}\right)\right) \alpha\left(P, q^{\prime}\right) \\
& \\
&\left.\quad+C_{2}\left(P^{\prime}, P-q_{1}, q_{2}\right)+C_{2}\left(P_{1}^{\prime}, q_{2}, q\right)\right) \alpha\left(P^{\prime}, q_{1}\right) \alpha\left(P-q_{1}, P^{\prime}, q_{2}\right)
\end{aligned}
$$

To write this equations in position space we take following strategy: express every vertex factor in terms of momentums of two point function $\alpha(P, q)$ and gauge boson propagator

$$
G(P, q)=\frac{(2 \pi)^{3} \delta^{(3)}(P)}{q_{-}} \Longrightarrow G(x, y)=\frac{i \delta\left(x^{3}-y^{3}\right)}{2 \pi(x-y)_{-}} \equiv G(x-y)
$$

and use the mapping mentioned in appendix A to convert them to derivatives in position space. These manipulations lead to the equations presented in (5.5), (5.7), (5.8), (5.9) and (5.10).

\footnotetext{
${ }^{50}$ Note that expressions given for $C_{1}, C_{2}$ is not unique as the integrand multiplying these has certain symmetries. For example $C_{1}\left(P, q_{1}, q_{2}\right)$ multiples $\chi\left(P, q_{1}\right) \chi\left(-P, q_{2}\right)$ which is invariant under simultaneous $q_{1} \leftrightarrow q_{2}, P \rightarrow-P$ exchange and therefore addition of any expression to $C_{1}$ which is 'odd' under this exchange integrates to zero.
} 


\section{Reduced set of bosonic gap equations}

In this appendix we simplify gap equation for bosons using specific form of the self-energy and propagators in presence of a uniform magnetic field. To do so we will need various properties of gauge field propagator given in (4.11) that we list below

$$
G(-x)=-G(x), \quad \partial_{x^{-}} G(x)=i \delta^{3}(x), \quad G(x)(G(y)+G(x-y))=G(x-y) G(y)
$$

Before proceeding, we introduce few notations

$$
\left(f_{1} f_{2}\right)(x)=f_{1}(x) f_{2}(x), \quad(\partial f)(x)=\partial_{x^{-}} f(x)
$$

We plug the form of $\alpha, \Sigma$ given in (2.17) into the gap equations presented in (5.5), (5.7), (5.8), (5.9) and (5.10). Below we present reduced part of each of the expressions.

$$
\begin{aligned}
& \left.\Sigma_{2 A}\left(x_{T}, x_{R}\right)\right|_{\text {red }}=4 \pi^{2} \lambda_{B}^{2}\left(\left(x_{6}+1\right) \delta^{3}\left(x_{R}\right) \alpha_{R}\left(x_{R}\right)^{2}\right. \\
& +2 D_{R}\left(\alpha_{R} \partial G *_{b} \alpha_{R} G\right)\left(x_{R}\right) \\
& \left.-\left(D_{R}+D_{T}\right)\left(D_{R}-D_{T}\right)\left(G \alpha_{R} *_{b} G \alpha_{R}\right)\left(x_{R}\right)\right) \\
& \left.\Sigma_{2 B}\left(x_{T}, x_{R}\right)\right|_{\text {red }}=4 \pi^{2} \lambda_{B}^{2}\left(x_{6} \alpha_{R}\left(x_{R}\right)^{2} \delta^{(3)}\left(x_{R}\right)\right. \\
& \left.+\left(D_{R}+D_{T}\right)\left(D_{R}-D_{T}\right)\left(G\left(\alpha_{R} *_{b} \alpha_{R} G\right)\right)\left(x_{R}\right)\right) \\
& \left.+2\left(D_{R}+D_{T}\right) D_{T}\left(G\left(\alpha_{R} *_{b} \alpha_{R} G\right)\right)\left(x_{R}\right)\right) \\
& -\left(D_{R}-D_{T}\right) 2 b \int d^{3} z x_{R_{1},-} \alpha_{R}\left(x_{1, R}\right) G\left(x_{R}\right) \alpha_{R}\left(x_{2, R}\right) G\left(x_{2, R}\right) e^{-\frac{i b}{2} \epsilon_{i j} x_{1, R}^{i} x_{2, R}^{j}} \\
& -2 D_{T} 2 b \int d^{3} z x_{R_{1},-} \alpha_{R}\left(x_{1, R}\right) G\left(x_{R}\right) \alpha_{R}\left(x_{2, R}\right) G\left(x_{2, R}\right) e^{-\frac{i b}{2} \epsilon_{i j} x_{1, R}^{i} x_{2, R}^{j}} \\
& -\left(D_{R}-D_{T}\right)\left(G \alpha_{R} *_{b} \alpha_{R} \partial G\right)\left(x_{R}\right)-2 D_{T}\left(G \alpha_{R} *_{b} \alpha_{R} \partial G\right)\left(x_{R}\right) \\
& +2 \int d^{3} z \alpha_{R}\left(x_{1, R}\right) \partial_{x_{R}^{-}}^{2} G\left(x_{R}\right) \alpha_{R}\left(x_{2, R}\right) G\left(x_{2, R}\right) e^{-\frac{i b}{2} \epsilon_{i j} x_{1, R}^{i} x_{2, R}^{j}} \\
& +\int d^{3} z \alpha_{R}\left(x_{1, R}\right) \partial_{x_{R}^{-}} G\left(x_{R}\right) \alpha_{R}\left(x_{2, R}\right) \partial_{x_{2, R}^{-}} G\left(x_{2, R}\right) e^{-\frac{i b}{2} \epsilon_{i j} x_{1, R}^{i} x_{2, R}^{j}} \\
& \left.+2 b \int d^{3} z x_{R_{1},-} \alpha_{R}\left(x_{1, R}\right) \partial_{x_{R}^{-}} G\left(x_{R}\right) \alpha_{R}\left(x_{2, R}\right) G\left(x_{2, R}\right)\right) e^{-\frac{i b}{2} \epsilon_{i j} x_{1, R}^{i} x_{2, R}^{j}} \\
& \Sigma_{2 B^{\prime}}\left(x_{T}, x_{R}\right)=4 \pi^{2} \lambda_{B}^{2}\left(x_{6} \alpha_{R}\left(x_{R}\right)^{2} \delta^{(3)}\left(x_{R}\right)\right. \\
& \left.+\left(D_{R}+D_{T}\right)\left(D_{R}-D_{T}\right)\left(G\left(\alpha_{R} G *_{b} \alpha_{R}\right)\right)\left(x_{R}\right)\right) \\
& -\left(D_{R}-D_{T}\right) 2 b \int d^{3} z x_{R_{1},-} \alpha_{R}\left(x_{1, R}\right) G\left(x_{R}\right) \alpha_{R}\left(x_{2, R}\right) G\left(x_{1, R}\right) e^{-\frac{i b}{2} \epsilon_{i j} x_{1, R}^{i} x_{2, R}^{j}} \\
& -\left(D_{R}-D_{T}\right)\left(\partial G \alpha_{R} *_{b} \alpha_{R} G\right)\left(x_{R}\right) \\
& +2 \int d^{3} z \alpha_{R}\left(x_{1, R}\right) \partial_{x_{R}^{-}}^{2} G\left(x_{R}\right) \alpha_{R}\left(x_{2, R}\right) G\left(x_{1, R}\right) e^{-\frac{i b}{2} \epsilon_{i j} x_{1, R}^{i} x_{2, R}^{j}} \\
& +\int d^{3} z \alpha_{R}\left(x_{1, R}\right) \partial_{x_{R}^{-}} G\left(x_{R}\right) \alpha_{R}\left(x_{2, R}\right) \partial_{x_{2, R}^{-}} G\left(x_{1, R}\right) e^{-\frac{i b}{2} \epsilon_{i j} x_{1, R}^{i} x_{2, R}^{j}} \\
& \left.+2 b \int d^{3} z x_{R_{1},-} \alpha_{R}\left(x_{1, R}\right) \partial_{x_{R}^{-}} G\left(x_{R}\right) \alpha_{R}\left(x_{2, R}\right) G\left(x_{1, R}\right)\right) e^{-\frac{i b}{2} \epsilon_{i j} x_{1, R}^{i} x_{2, R}^{j}}
\end{aligned}
$$


Where we have defined

$$
D_{R}=2\left(-\frac{b}{2} X_{T,-}+\partial_{X_{R}^{-}}\right), D_{T}=2\left(\frac{b}{2} X_{R,-}+\partial_{X_{T}^{-}}\right)
$$

Now we present few identities that will we will need to simplify above equations. These rely crucially on properties of twisted convolution and properties given in (I.7) of gauge boson propagator. For any function $\alpha(x)$, it can be shown that

$$
\begin{aligned}
& \left.\left(\left(\alpha \partial G *_{b} \alpha\right) G\right)(x-y)=\left(\alpha \partial G *_{b} \alpha G\right)(x-y)\right) \\
& \left(G\left(\alpha *_{b} \alpha \partial G\right)\right)(x-y)=\left(\alpha G *_{b} \alpha \partial G\right)(x-y) \\
& \left(\partial G\left(\alpha *_{b} \alpha G\right)\right)(x-y)=\left(\left(\alpha G *_{b} \alpha\right) \partial G\right)(x-y)=0 \\
& \left(G\left(\alpha *_{b} \alpha G\right)+\left(\alpha G *_{b} \alpha\right) G\right)(x-y)=\left(\alpha G *_{b} \alpha G\right)(x-y) \\
& \left(\partial^{2} G\left(\alpha *_{b} \alpha G\right)\right)(x-y)+\frac{1}{2}\left(\partial G \alpha *_{b} \alpha \partial G\right)(x-y)=+\frac{b}{2} \int \partial G(x-y) \alpha(x-y-z) \alpha(z) G(z) z_{-} d^{3} z \\
& \left(\partial^{2} G\left(\alpha G *_{b} \alpha\right)\right)(x-y)+\frac{1}{2}\left(\partial G \alpha *_{b} \alpha \partial G\right)(x-y)=-\frac{b}{2} \int \partial G(x-y) \alpha(x-y-z) \alpha(z) G(z) z_{-} d^{3} z \\
& \int d^{3} z x_{R_{1},-} \alpha_{R}\left(x_{1, R}\right) \partial_{x_{R}^{-}} G\left(x_{R}\right) \alpha_{R}\left(x_{2, R}\right)\left(G\left(x_{1, R}\right)+G\left(x_{2, R}\right)\right)=0 \\
& \int d^{3} z x_{R_{1},-} \alpha_{R}\left(x_{1, R}\right) G\left(x_{R}\right) \alpha_{R}\left(x_{2, R}\right)\left(G\left(x_{1, R}\right)\right. \\
& \left.+G\left(x_{2, R}\right)\right) e^{-\frac{i b}{2} \epsilon_{i j} x_{1, R}^{i} x_{2, R}^{j}}=x_{R,-}\left(G\left(\alpha *_{b} \alpha G\right)\right)\left(x_{R}\right)
\end{aligned}
$$

Due to 1st, 2nd identity in (I.7), 2nd line of (I.3), 6th line of (I.4) and 4th line of (I.5) sums to zero. Due to 4th identity in (I.7), 3rd line of (I.3), 2nd line of (I.4) and 2nd line of (I.5) sums to zero. Due to 5th, 6th identity in (I.7), 7th, 8th line of (I.4) and 5th,6th line of (I.5) sums to zero. Due to 7th identity in (I.7), last line of (I.4) and last line of (I.5) sums to zero.

Now we use last identity in (I.7) to simplify the following

$$
\begin{aligned}
& \left.2\left(D_{R}+D_{T}\right) D_{T}\left(G\left(\alpha_{R} *_{b} \alpha_{R} G\right)\right)\left(x_{R}\right)\right) \\
& -\left(D_{R}-D_{T}\right) 2 b \int d^{3} z x_{R_{1},-} \alpha_{R}\left(x_{1, R}\right) G\left(x_{R}\right) \alpha_{R}\left(x_{2, R}\right) G\left(x_{2, R}\right) e^{-\frac{i b}{2} \epsilon_{i j} x_{1, R}^{i} x_{2, R}^{j}} \\
& -\left(D_{R}-D_{T}\right) 2 b \int d^{3} z x_{R_{1},-} \alpha_{R}\left(x_{1, R}\right) G\left(x_{R}\right) \alpha_{R}\left(x_{2, R}\right) G\left(x_{1, R}\right) e^{-\frac{i b}{2} \epsilon_{i j} x_{1, R}^{i} x_{2, R}^{j}} \\
& \left.\quad=\left(D_{R}+D_{T}\right) 2 b x_{R,-}\left(G\left(\alpha_{R} *_{b} \alpha_{R} G\right)\right)\left(x_{R}\right)\right)-\left(D_{R}-D_{T}\right) 2 b x_{R,-}\left(G\left(\alpha_{R} *_{b} \alpha_{R} G\right)\right)\left(x_{R}\right) \\
& \left.\quad=2 D_{T} 2 b x_{R,-}\left(G\left(\alpha_{R} *_{b} \alpha_{R} G\right)\right)\left(x_{R}\right)\right) \\
& \left.\quad=\left(2 b x_{R,-}\right)^{2}\left(G\left(\alpha_{R} *_{b} \alpha_{R} G\right)\right)\left(x_{R}\right)\right)
\end{aligned}
$$

The only remaining complex term can be simplified as follows

$$
\begin{aligned}
& -2 D_{T} 2 b \int d^{3} z x_{R_{1},-} \alpha_{R}\left(x_{1, R}\right) G\left(x_{R}\right) \alpha_{R}\left(x_{2, R}\right) G\left(x_{2, R}\right) e^{-\frac{i b}{2} \epsilon_{i j} x_{1, R}^{i} x_{2, R}^{j}} \\
& =-2 b x_{R,-} 2 b G\left(x_{R}\right) \int d^{3} z\left(x_{R}-z\right)_{-} \alpha_{R}\left(x_{R}-z\right) \alpha_{R}(z) G(z) e^{-\frac{i b}{2} \epsilon_{i j} x_{1, R}^{i} x_{2, R}^{j}}
\end{aligned}
$$




$$
\begin{aligned}
= & -\left(2 b x_{R,-}\right)^{2} G\left(x_{R}\right) \int d^{3} z \alpha_{R}\left(x_{R}-z\right) \alpha_{R}(z) G(z) e^{-\frac{i b}{2} \epsilon_{i j} x_{1, R}^{i} x_{2, R}^{j}} \\
& -\left(\frac{b}{\pi}\right)^{2} \delta\left(x_{R}^{3}\right) \int d^{3} z \alpha_{R}\left(x_{R}-z\right) \alpha_{R}(z) \delta\left(z^{3}\right) e^{-\frac{i b}{2} \epsilon_{i j} x_{1, R}^{i} x_{2, R}^{j}} \\
= & \left.-\left(2 b x_{R,-}\right)^{2}\left(G\left(\alpha_{R} *_{b} \alpha_{R} G\right)\right)\left(x_{R}\right)\right) \\
& -\left(\frac{b}{\pi}\right)^{2} \int d^{3} z \delta\left(x_{R}^{3}-z^{3}\right) \alpha_{R}\left(x_{R}-z\right) \alpha_{R}(z) \delta\left(z^{3}\right) e^{-\frac{i b}{2} \epsilon_{i j} x_{1, R}^{i} x_{2, R}^{j}}
\end{aligned}
$$

Adding the final results of (I.8) and (I.9) to the (uncanceled) expressions on the first lines of the r.h.s. of (I.3), (I.4), (I.5) we obtain two loop contribution to reduced self-energy to be

$4 \pi^{2} \lambda_{B}^{2}\left(3 x_{6}+1\right) \delta^{3}\left(x_{R}\right) \alpha_{R}\left(x_{R}\right)^{2}-\left(2 b \lambda_{B}\right)^{2} \int d^{3} z \delta\left(x_{R}^{3}-z^{3}\right) \alpha_{R}\left(x_{R}-z\right) \alpha_{R}(z) \delta\left(z^{3}\right) e^{-\frac{i b}{2} \epsilon_{i j} x_{1, R}^{i} x_{2, R}^{j}}$

\section{J Zero magnetic field limit}

In this appendix we discuss how to take $b \rightarrow 0$ limit carefully. Note that this limit is bit special - in absence of magnetic field energy levels of a free theory has a continuous spectrum, whereas for any non-zero magnetic field that becomes discrete. Therefore $b=0$ discussion is an averaged out version of many very closely spaced levels that are present for small values of $b$ as we will show below. We will discuss only the case zero chemical potential.

Fermions. Note that significant contribution comes only from $n \rightarrow \infty$ part of summations, such that $w=b n$ is kept fixed. In this limit $\chi_{n}$ as obtained in (4.52) simplifies to

$$
\chi_{n}=\frac{1}{2\left(c_{F}^{2}+2 w\right)^{1 / 2}}
$$

Gap equations (4.46) becomes

$$
\begin{aligned}
\frac{\partial}{\partial w}\left(\Sigma_{R, I, n}\right) & =2 \lambda_{F} \chi_{n} \\
\frac{\partial}{\partial w}\left(\sqrt{w} \Sigma_{R,+, n}\right) & =-2 i \lambda_{F} K_{R, I, n} \chi_{n}
\end{aligned}
$$

First of these gap equations is solved by it's integrated form (4.30)

$$
\Sigma_{R, I, n}(w)=-2 \lambda_{F} \int_{w}^{\infty} \chi_{n}\left(w^{\prime}\right) d w^{\prime}
$$

Where above equation is regularized by dimensional regularization on the quantity $c_{F}^{2}+2 w$, i.e., effectively on $\omega$ integration that we performed previously. These gives

$$
\begin{aligned}
\Sigma_{R, I, n}(w) & =\lambda_{F}\left(c_{F}^{2}+2 w\right)^{1 / 2} \\
\Sigma_{R,+, n}(w) & =-\frac{i}{2 \sqrt{w}}\left(\Sigma_{R, I, n}(w)^{2}-\Sigma_{R, I, n}(w=0)^{2}+2 m_{F}\left(\Sigma_{R, I, n}(w)-\Sigma_{R, I, n}(w=0)\right)\right)
\end{aligned}
$$


Plugging these two into (J.1) we get

$$
c_{F}^{2}=\left(m_{F}+\Sigma_{R, I, n}(w=0)\right)^{2}
$$

Summing components given in (J.4) with corresponding basis functions listed in (D.11) we get corresponding self-energies in position space

$$
\begin{aligned}
\Sigma_{R, I}(\vec{x}) & =\sum_{n} \Sigma_{R, I, n}(\vec{x}) e_{n, n}(\vec{x}) \\
& \rightarrow \int \frac{d w}{2 \pi} \lim _{n \rightarrow \infty} L_{n}^{0}\left(\frac{1}{n} \frac{w r^{2}}{2}\right) \Sigma_{R, I, n} \\
& =\int \frac{p_{s} d p_{s}}{2 \pi} J_{0}\left(p_{s} r\right) \lambda_{F}\left(c_{F}^{2}+p_{s}^{2}\right)^{1 / 2}
\end{aligned}
$$

Here we have changed variables $w=\frac{p_{s}^{2}}{2}$, and used following identity of Laguerre polynomial

$$
\lim _{n \rightarrow \infty} \frac{1}{n^{l}} L_{n}^{l}\left(\frac{x}{n}\right)=\frac{1}{x^{l}} J_{l}(2 \sqrt{x})
$$

To compare our results with previous works on the subject we switch to momentum space defined by

$$
\tilde{F}(\vec{p})=\int d^{2} x F(\vec{x}) e^{-i \vec{p} \cdot \vec{x}}
$$

A basic property that relates position and momentum space is that the function which has ${ }^{51}$

$$
\tilde{F}(\vec{p})=e^{i m \theta} f\left(p_{s}\right)
$$

in position space is given by

$$
F(\vec{x})=\int \frac{p_{s} d p_{s}}{2 \pi} f\left(p_{s}\right) J_{|m|}\left(p_{s} r\right) e^{i m \phi}
$$

Comparing above obtained form of $\Sigma_{R, I}$ and similar considerations for $\Sigma_{R,+}$, dictates that solutions given in (J.4) has following form in momentum space

$$
\begin{aligned}
\Sigma_{R, I}(\vec{p}) & =\lambda_{F}\left(c_{F}^{2}+p_{s}^{2}\right)^{1 / 2} \\
\Sigma_{R,+}(\vec{p}) & =\frac{p_{+}}{p_{s}^{2}}\left(c_{F}^{2}-\left(m_{F}+\Sigma_{R, I}(\vec{p})\right)^{2}\right) \\
c_{F}^{2} & =\left(m_{F}+\lambda_{F}\left|c_{F}\right|\right)^{2}
\end{aligned}
$$

(J.11) agrees with the solution of the zero magnetic field gap equations presented in $[4,20$, $28,32]$.

\footnotetext{
${ }^{51}$ We are using following notation

$$
p^{ \pm}=\frac{p_{s}}{\sqrt{2}} e^{ \pm i \theta}, x^{ \pm}=\frac{r}{\sqrt{2}} e^{ \pm i \phi}
$$


Bosons. First note that (5.18), (5.19) suggests that

$$
\zeta_{+}^{B}(n)=\zeta_{-}^{B}(n)=c_{B}^{2}+2 b n
$$

in the limit we are considering. On the other hand (5.24) gives

$$
\int \frac{b d n}{\sqrt{c_{B}^{2}+2 b n}}=-m_{B}^{\mathrm{cri}} \Longrightarrow\left|c_{B}\right|=m_{B}^{\mathrm{cri}}
$$

Finally performing an analysis very similar to that for the fermions (see around (5.15)) gives

$$
\Sigma(\vec{p})=\left(m_{B}^{\text {cri }}\right)^{2}-m_{B}^{2}
$$

in agreement with the gap equations presented in $[8,20,28,32]$.

Open Access. This article is distributed under the terms of the Creative Commons Attribution License (CC-BY 4.0), which permits any use, distribution and reproduction in any medium, provided the original author(s) and source are credited.

\section{References}

[1] I.R. Klebanov and A.M. Polyakov, AdS dual of the critical $O(N)$ vector model, Phys. Lett. B $\mathbf{5 5 0}$ (2002) 213 [hep-th/0210114] [INSPIRE].

[2] E. Sezgin and P. Sundell, Massless higher spins and holography, Nucl. Phys. B 644 (2002) 303 [Erratum ibid. B 660 (2003) 403] [hep-th/0205131] [INSPIRE].

[3] S. Giombi and X. Yin, Higher Spin Gauge Theory and Holography: The Three-Point Functions, JHEP 09 (2010) 115 [arXiv:0912.3462] [INSPIRE].

[4] S. Giombi, S. Minwalla, S. Prakash, S.P. Trivedi, S.R. Wadia and X. Yin, Chern-Simons Theory with Vector Fermion Matter, Eur. Phys. J. C 72 (2012) 2112 [arXiv:1110.4386] [INSPIRE].

[5] C.-M. Chang, S. Minwalla, T. Sharma and X. Yin, ABJ Triality: from Higher Spin Fields to Strings, J. Phys. A 46 (2013) 214009 [arXiv:1207.4485] [InSPIRE].

[6] J. Maldacena and A. Zhiboedov, Constraining Conformal Field Theories with A Higher Spin Symmetry, J. Phys. A 46 (2013) 214011 [arXiv:1112.1016] [InSPIRE].

[7] J. Maldacena and A. Zhiboedov, Constraining conformal field theories with a slightly broken higher spin symmetry, Class. Quant. Grav. 30 (2013) 104003 [arXiv:1204.3882] [INSPIRE].

[8] O. Aharony, G. Gur-Ari and R. Yacoby, Correlation Functions of Large N Chern-Simons-Matter Theories and Bosonization in Three Dimensions, JHEP 12 (2012) 028 [arXiv: 1207.4593] [INSPIRE].

[9] G. Gur-Ari and R. Yacoby, Correlators of Large N Fermionic Chern-Simons Vector Models, JHEP 02 (2013) 150 [arXiv: 1211.1866] [INSPIRE].

[10] O. Aharony, Baryons, monopoles and dualities in Chern-Simons-matter theories, JHEP 02 (2016) 093 [arXiv: 1512.00161] [INSPIRE].

[11] N. Seiberg, T. Senthil, C. Wang and E. Witten, A Duality Web in 2+1 Dimensions and Condensed Matter Physics, Annals Phys. 374 (2016) 395 [arXiv:1606.01989] [InSPIRE]. 
[12] A. Karch and D. Tong, Particle-Vortex Duality from 3d Bosonization, Phys. Rev. X 6 (2016) 031043 [arXiv: 1606.01893] [INSPIRE].

[13] J. Murugan and H. Nastase, Particle-vortex duality in topological insulators and superconductors, JHEP 05 (2017) 159 [arXiv: 1606.01912] [INSPIRE].

[14] P.-S. Hsin and N. Seiberg, Level/rank Duality and Chern-Simons-Matter Theories, JHEP 09 (2016) 095 [arXiv: 1607.07457] [INSPIRE].

[15] O. Aharony, F. Benini, P.-S. Hsin and N. Seiberg, Chern-Simons-matter dualities with SO and USp gauge groups, JHEP 02 (2017) 072 [arXiv:1611.07874] [INSPIRE].

[16] J. Gomis, Z. Komargodski and N. Seiberg, Phases Of Adjoint $Q C D_{3}$ And Dualities, SciPost Phys. 5 (2018) 007 [arXiv:1710.03258] [INSPIRE].

[17] C. Córdova, P.-S. Hsin and N. Seiberg, Global Symmetries, Counterterms and Duality in Chern-Simons Matter Theories with Orthogonal Gauge Groups, SciPost Phys. 4 (2018) 021 [arXiv: 1711.10008] [INSPIRE].

[18] C. Córdova, P.-S. Hsin and N. Seiberg, Time-Reversal Symmetry, Anomalies and Dualities in $(2+1) d$, SciPost Phys. 5 (2018) 006 [arXiv: 1712.08639] [INSPIRE].

[19] M.A. Metlitski, A. Vishwanath and C. Xu, Duality and bosonization of (2+1)-dimensional Majorana fermions, Phys. Rev. B 95 (2017) 205137 [arXiv: 1611.05049] [INSPIRE].

[20] S. Jain, M. Mandlik, S. Minwalla, T. Takimi, S.R. Wadia and S. Yokoyama, Unitarity, Crossing Symmetry and Duality of the S-matrix in large $N$ Chern-Simons theories with fundamental matter, JHEP 04 (2015) 129 [arXiv: 1404.6373] [INSPIRE].

[21] Y. Dandekar, M. Mandlik and S. Minwalla, Poles in the S-Matrix of Relativistic Chern-Simons Matter theories from Quantum Mechanics, JHEP 04 (2015) 102 [arXiv: 1407.1322] [INSPIRE].

[22] K. Inbasekar, S. Jain, S. Mazumdar, S. Minwalla, V. Umesh and S. Yokoyama, Unitarity, crossing symmetry and duality in the scattering of $\mathcal{N}=1$ SUSY matter Chern-Simons theories, JHEP 10 (2015) 176 [arXiv:1505.06571] [INSPIRE].

[23] S. Yokoyama, Scattering Amplitude and Bosonization Duality in General Chern-Simons Vector Models, JHEP 09 (2016) 105 [arXiv: 1604.01897] [INSPIRE].

[24] K. Inbasekar, S. Jain, P. Nayak and V. Umesh, All tree level scattering amplitudes in Chern-Simons theories with fundamental matter, Phys. Rev. Lett. 121 (2018) 161601 [arXiv: 1710.04227] [INSPIRE].

[25] K. Inbasekar et al., Dual superconformal symmetry of $\mathcal{N}=2$ Chern-Simons theory with fundamental matter at large $N$, JHEP 06 (2019) 016 [arXiv:1711.02672] [INSPIRE].

[26] S. Jain, S.P. Trivedi, S.R. Wadia and S. Yokoyama, Supersymmetric Chern-Simons Theories with Vector Matter, JHEP 10 (2012) 194 [arXiv:1207.4750] [INSPIRE].

[27] S. Yokoyama, Chern-Simons-Fermion Vector Model with Chemical Potential, JHEP 01 (2013) 052 [arXiv: 1210.4109] [INSPIRE].

[28] O. Aharony, S. Giombi, G. Gur-Ari, J. Maldacena and R. Yacoby, The Thermal Free Energy in Large N Chern-Simons-Matter Theories, JHEP 03 (2013) 121 [arXiv:1211.4843] [INSPIRE].

[29] S. Jain, S. Minwalla, T. Sharma, T. Takimi, S.R. Wadia and S. Yokoyama, Phases of large N vector Chern-Simons theories on $S^{2} \times S^{1}$, JHEP 09 (2013) 009 [arXiv:1301.6169] [INSPIRE]. 
[30] T. Takimi, Duality and higher temperature phases of large $N$ Chern-Simons matter theories on $S^{2} \times S^{1}$, JHEP 07 (2013) 177 [arXiv: 1304.3725] [INSPIRE].

[31] S. Yokoyama, A Note on Large $N$ Thermal Free Energy in Supersymmetric Chern-Simons Vector Models, JHEP 01 (2014) 148 [arXiv:1310.0902] [INSPIRE].

[32] S. Jain, S. Minwalla and S. Yokoyama, Chern Simons duality with a fundamental boson and fermion, JHEP 11 (2013) 037 [arXiv:1305.7235] [INSPIRE].

[33] S. Minwalla and S. Yokoyama, Chern Simons Bosonization along RG Flows, JHEP 02 (2016) 103 [arXiv: 1507.04546] [INSPIRE].

[34] G. Gur-Ari and R. Yacoby, Three Dimensional Bosonization From Supersymmetry, JHEP 11 (2015) 013 [arXiv : 1507.04378] [INSPIRE].

[35] M. Geracie, M. Goykhman and D.T. Son, Dense Chern-Simons Matter with Fermions at Large N, JHEP 04 (2016) 103 [arXiv: 1511.04772] [INSPIRE].

[36] D. Radičević, Disorder Operators in Chern-Simons-Fermion Theories, JHEP 03 (2016) 131 [arXiv: 1511.01902] [INSPIRE].

[37] S. Choudhury et al., Bose-Fermi Chern-Simons Dualities in the Higgsed Phase, JHEP 11 (2018) 177 [arXiv: 1804.08635] [INSPIRE].

[38] O. Aharony, S. Jain and S. Minwalla, Flows, Fixed Points and Duality in Chern-Simons-matter theories, JHEP 12 (2018) 058 [arXiv: 1808. 03317] [INSPIRE].

[39] A. Dey, I. Halder, S. Jain, L. Janagal, S. Minwalla and N. Prabhakar, Duality and an exact Landau-Ginzburg potential for quasi-bosonic Chern-Simons-Matter theories, JHEP 11 (2018) 020 [arXiv: 1808.04415] [INSPIRE].

[40] A. Bedhotiya and S. Prakash, A test of bosonization at the level of four-point functions in Chern-Simons vector models, JHEP 12 (2015) 032 [arXiv: 1506.05412] [INSPIRE].

[41] G.J. Turiaci and A. Zhiboedov, Veneziano Amplitude of Vasiliev Theory, JHEP 10 (2018) 034 [arXiv: 1802.04390] [INSPIRE].

[42] O. Aharony, L.F. Alday, A. Bissi and R. Yacoby, The Analytic Bootstrap for Large $N$ Chern-Simons Vector Models, JHEP 08 (2018) 166 [arXiv:1805.04377] [INSPIRE].

[43] S. Kim, The complete superconformal index for $N=6$ Chern-Simons theory, Nucl. Phys. B 821 (2009) 241 [Erratum ibid. B 864 (2012) 884] [arXiv: 0903.4172] [INSPIRE].

[44] A. Kapustin, B. Willett and I. Yaakov, Exact Results for Wilson Loops in Superconformal Chern-Simons Theories with Matter, JHEP 03 (2010) 089 [arXiv:0909.4559] [INSPIRE].

[45] N. Drukker, M. Mariño and P. Putrov, Nonperturbative aspects of ABJM theory, JHEP 11 (2011) 141 [arXiv:1103.4844] [INSPIRE].

[46] Y. Imamura and S. Yokoyama, Index for three dimensional superconformal field theories with general R-charge assignments, JHEP 04 (2011) 007 [arXiv:1101.0557] [INSPIRE].

[47] R. Gopakumar, S. Minwalla and A. Strominger, Noncommutative solitons, JHEP 05 (2000) 020 [hep-th/0003160] [INSPIRE].

[48] M. Aganagic, R. Gopakumar, S. Minwalla and A. Strominger, Unstable solitons in noncommutative gauge theory, JHEP 04 (2001) 001 [hep-th/0009142] [INSPIRE].

[49] N. Seiberg and E. Witten, String theory and noncommutative geometry, JHEP 09 (1999) 032 [hep-th/9908142] [INSPIRE]. 
[50] S. Minwalla, M. Van Raamsdonk and N. Seiberg, Noncommutative perturbative dynamics, JHEP 02 (2000) 020 [hep-th/9912072] [INSPIRE].

[51] E. Witten, Quantum Field Theory and the Jones Polynomial, Commun. Math. Phys. 121 (1989) 351 [INSPIRE].

[52] I. Halder, L. Janagal, S. Minwalla, N. Prabhakar, D. Radičević and T. Sharma, to appear.

[53] S.-S. Lee, Low energy effective theory of Fermi surface coupled with U(1) gauge field in 2+1 dimensions, Phys. Rev. B 80 (2009) 165102 [arXiv:0905.4532] [INSPIRE]. 